\title{
Synthetic Glycosphingolipids for Live-Cell Labeling
}

Martin Dauner, ${ }^{\#, \dagger}$ Ellen Batroff,,${ }^{\sharp \dagger}$ Verena Bachmann, ${ }^{\ddagger}$ Christof R. Hauck, ${ }^{\ddagger}$ and Valentin Wittmann*,†

${ }^{\dagger}$ University of Konstanz, Department of Chemistry and Konstanz Research School Chemical Biology (KoRS-CB), 78457 Konstanz, Germany. Phone: +49-7531-88-4572. Fax: +49-753188-4573. E-mail: mail@valentin-wittmann.de.

$\$$ University of Konstanz, Department of Biology and Konstanz Research School Chemical Biology (KoRS-CB), 78457 Konstanz, Germany

\# These authors contributed equally.

\section{Table of Contents}

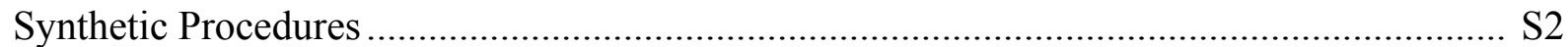

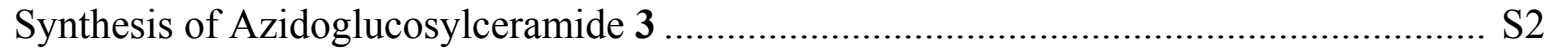

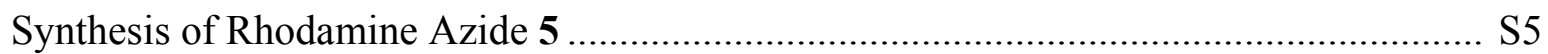

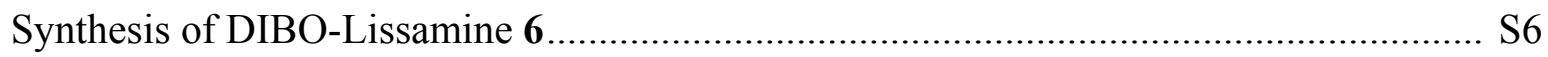

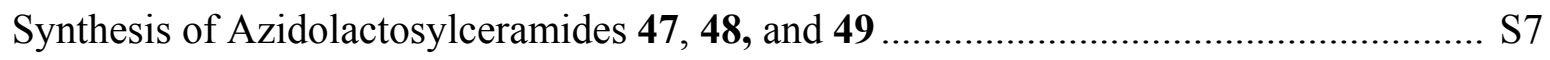

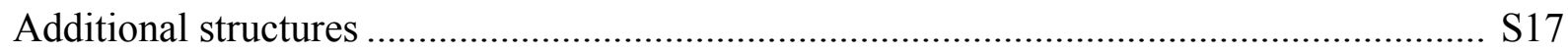

Incorporation of Azidolactosylceramides $\mathbf{4 7}$ and $\mathbf{4 8}$ at Different Temperatures ................. S18

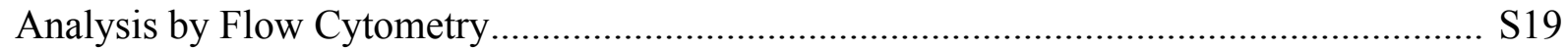

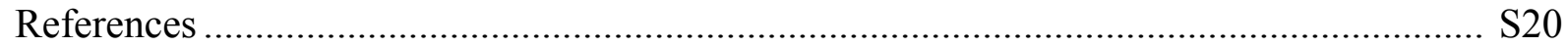

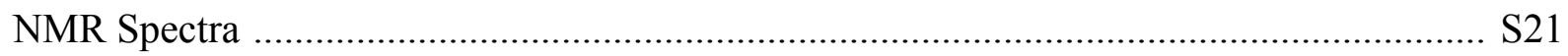




\section{Synthetic Procedures}

Synthesis of Azidoglucosylceramide 3

\section{$N-[(1 S, 2 R, 3 E)-2-(B e n z o y l o x y)-1-[($ triphenylmethoxy)methyl]-3-heptadecen-1-yl]- tetradecanamide (25)}

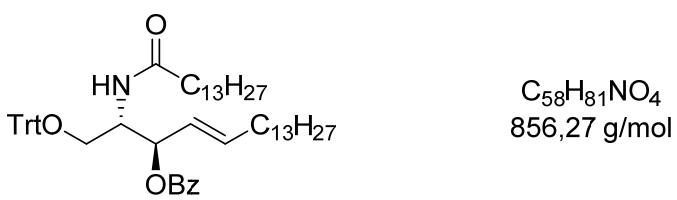

$150 \mathrm{mg}(0.29 \mathrm{mmol})$ ceramide 17 and $98 \mathrm{mg}(0.35 \mathrm{mmol})$ trityl chloride were dissolved in 3 $\mathrm{mL}$ pyridine/DCM/THF 1:1:1. The reaction was stirred for $16 \mathrm{~h}$ at room temperature. The solvent was removed under reduced pressure. The remaining material was dissolved in a small amount of DCM and washed with saturated $\mathrm{NaHCO}_{3}$ solution and water. The organic phase was dried with $\mathrm{MgSO}_{4}$ and evaporated under reduced pressure. The crude product (21) was dissolved in $3 \mathrm{~mL}$ toluene/pyridine $4: 1$. Benzoyl chloride ( $70 \mu \mathrm{L}, 0.6 \mathrm{mmol})$ was added, and the mixture was stirred for $16 \mathrm{~h}$ at room temperature. A small amount of DCM was added, and the mixture was washed with saturated $\mathrm{NaHCO}_{3}$ solution. The organic phase was dried with $\mathrm{MgSO}_{4}$ and evaporated under reduced pressure. The crude product was purified by silica gel chromatography (eluent petroleum ether/ethyl acetate 9:1). 25 was obtained as a colorless solid (176 mg, $0.21 \mathrm{mmol}, 72 \%$ over two steps).

TLC: $R_{f}=0.43$ (eluent petroleum ether/ethyl acetate $4: 1$ )

${ }^{1} \mathbf{H}$ NMR (250 MHz, $\mathrm{CDCl}_{3}$ ): $\delta=8.02-7.97$ (m, $3 \mathrm{H}$, aromat.), 7.88 (d, $J=7.1 \mathrm{~Hz}, 3 \mathrm{H}$, aromat.), $7.57-7.09$ (m, $15 \mathrm{H}$, aromat.), $5.89-5.78$ (m, $\left.2 \mathrm{H}, \mathrm{NH}, \mathrm{CH}=\mathrm{CHCH}_{2}\right), 5.65$ (' $\mathrm{t}$ ', $J=$ 7.6 Hz, CHOBz), 5.38 (dd, $\left.J=15.5,7.7 \mathrm{~Hz}, \mathrm{CH}=\mathrm{CHCH}_{2}\right), 4.45-4.37$ (m, $\left.1 \mathrm{H}, \mathrm{C} \underline{\mathrm{HNH}}\right), 3.38$ (dd, $J=9.6,3.9 \mathrm{~Hz}, 1 \mathrm{H}, \mathrm{CH}_{2} \mathrm{OTrt}$ ), $3.19-3.08$ (m, $\left.1 \mathrm{H}, \mathrm{CH}_{2} \mathrm{OTrt}\right), 2.08$ (t, $J=7.5 \mathrm{~Hz}, 2 \mathrm{H}$, $\left.\mathrm{C}(\mathrm{O}) \mathrm{CH}_{2}\right), 1.96-1.89\left(\mathrm{~m}, 2 \mathrm{H}, \mathrm{CH}=\mathrm{CHC}_{2}\right), 1.61-1.45\left(\mathrm{~m}, 2 \mathrm{H}, \mathrm{C}(\mathrm{O}) \mathrm{CH}_{2} \underline{\mathrm{C}}_{2}\right), 1.32$ $1.10(\mathrm{~m}, 42 \mathrm{H}, 21 \mathrm{x} \mathrm{CH}), 0.83$ (t, $\left.J=6.5 \mathrm{~Hz}, 6 \mathrm{H}, 2 \mathrm{x} \mathrm{CH}_{3}\right)$.

\section{$N$-[(1S,2R,3E)-2-(Benzoyloxy)-1-(hydroxymethyl)-3-heptadecen-1-yl]-tetradecanamide}

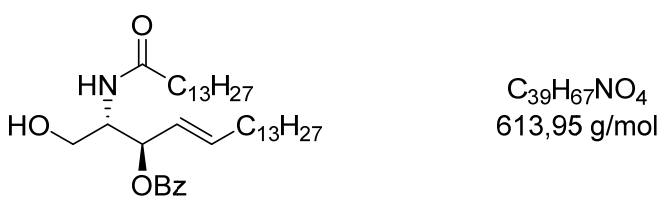

Ceramide 29 was synthesized as described for $\mathbf{2 3}$, starting from $176 \mathrm{mg}$ ceramide $25(0.21$ 
mmol). After purification by silica chromatography $94 \mathrm{mg} 29(0.15 \mathrm{mmol}, 74 \%)$ was obtained as colorless solid.

TLC: $R_{f}=0.26$ (eluent petroleum ether/ethyl acetate $1: 1$ )

${ }^{1} \mathbf{H}$ NMR (400 MHz, $\left.\mathrm{CDCl}_{3}\right): \delta=8.05-8.02$ (m, $2 \mathrm{H}$, aromat.), $7.58-7.55$ (m, $1 \mathrm{H}$, aromat.), $7.45-7.42$ (m, $2 \mathrm{H}$, aromat.), 6.22 (d, $J=8.5 \mathrm{~Hz}, 1 \mathrm{H}, \mathrm{NH}), 5.86$ (dt, $J=14.5,6.8$ $\left.\mathrm{Hz}, 1 \mathrm{H}, \mathrm{CH}=\mathrm{C}_{\mathbf{H C H}}\right), 5.55\left(\mathrm{dd}, J=14.6,7.4 \mathrm{~Hz}, 1 \mathrm{H}, \underline{\mathrm{C}} \underline{\mathrm{H}}=\mathrm{CHCH}_{2}\right), 5.51(\mathrm{~m}, 1 \mathrm{H}, \mathrm{CHOBz})$, 4.25 (m, $1 \mathrm{H}, \mathrm{C} \underline{\mathrm{HNH}}), 3.73\left(\mathrm{dd}, J=12.2,3.5 \mathrm{~Hz}, 1 \mathrm{H}, \underline{\mathrm{C}}_{2} \mathrm{OH}\right), 3.62(\mathrm{dd}, J=12.2,3.3 \mathrm{~Hz}, 1$ $\left.\mathrm{H}, \mathrm{C}_{2} \mathrm{OH}\right), 2.65$ (b, $\left.1 \mathrm{H}, \mathrm{OH}\right), 2.22-2.17\left(\mathrm{~m}, 2 \mathrm{H}, \mathrm{C}(\mathrm{O}) \mathrm{CH}_{2}\right), 2.03-1.99(\mathrm{~m}, 2 \mathrm{H}$, $\left.\mathrm{CH}=\mathrm{CHC}_{2}\right), 1.67-1.53\left(\mathrm{~m}, 2 \mathrm{H}, \mathrm{C}(\mathrm{O}) \mathrm{CH}_{2} \mathrm{C}_{2}\right), 1.37-1.16\left(\mathrm{~m}, 42 \mathrm{H}, 21 \times \mathrm{CH}_{2}\right), 0.89$ (t, $J$ $\left.=6.8 \mathrm{~Hz}, 6 \mathrm{H}, 2 \mathrm{x} \mathrm{CH}_{3}\right)$.

ESI-MS: $\quad$ calculated $\quad[\mathrm{M}+\mathrm{H}]^{+}=614.5,[\mathrm{M}+\mathrm{Na}]^{+}=636.5$ found $[\mathrm{M}+\mathrm{H}]^{+}=614.5,[\mathrm{M}+\mathrm{Na}]^{+}=636.6$

\section{(2S,3S,4E)-2-Tetradecanamido-3-(benzoyloxy)-4-octadecen-1-yl-2,3,4-tetra- $O$-acetyl-6-} azido-6-deoxy- $\beta$-D-glucopyranoside (35)

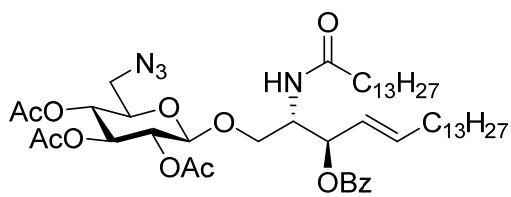

Glycolipid 35 was synthesized as described for 34, starting from $94 \mathrm{mg}(0.153 \mathrm{mmol})$ ceramide 29 and $87 \mathrm{mg}(0.18 \mathrm{mmol})$ trichloroacetimidate 33. After purification $28 \mathrm{mg}(0.03$ mmol, $20 \%$ ) of $\mathbf{3 5}$ was obtained as a colorless solid.

TLC: $R_{f}=0.59$ (eluent toluene/acetone $4: 1$ )

${ }^{1}$ H NMR (400 MHz, $\left.\mathrm{CDCl}_{3}\right): \delta=8.04-8.00$ (m, $2 \mathrm{H}$, aromat.), $7.55-7.52$ (m, $1 \mathrm{H}$, aromat.), $7.45-7.41$ (m, $2 \mathrm{H}$, aromat.), $5.90-5.81$ (m, $\left.1 \mathrm{H}, \mathrm{CH}=\mathrm{CHCH}_{2}\right), 5.76$ (d, $J=9.2$ $\mathrm{Hz}, 1 \mathrm{H}, \mathrm{NH}$ ), 5.53 ('t', $J=6.9 \mathrm{~Hz}, 1 \mathrm{H}, \mathrm{CHOBz}), 5.45$ (dd, $J=15.2,7.4 \mathrm{~Hz}, 1 \mathrm{H}$, $\left.\mathrm{C} \underline{\mathrm{H}}=\mathrm{CHCH}_{2}\right), 5.17$ ('t', $\left.J=9.5 \mathrm{~Hz}, 1 \mathrm{H}, \mathrm{H}-3\right), 4.97-4.90$ (m, $\left.2 \mathrm{H}, \mathrm{H}-2, \mathrm{H}-4\right), 4.50$ (d, $J=8.0$ $\mathrm{Hz}, 1 \mathrm{H}, \mathrm{H}-1), 4.50$ - 4.45 (m, $1 \mathrm{H}, \mathrm{C} \underline{\mathrm{HNH}}$ ), 4.04 (dd, $J=10.0,4.3 \mathrm{~Hz}, 1 \mathrm{H}, \mathrm{CH}_{2} \mathrm{OGlc}$ ), 3.68 (dd, $\left.J=10.2,4.3 \mathrm{~Hz}, 1 \mathrm{H}, \mathrm{CH}_{2} \mathrm{OGlc}\right), 3.67-3.61$ (m, $\left.1 \mathrm{H}, \mathrm{H}-5\right), 3.23$ (dd, $J=13.4,6.8 \mathrm{~Hz}, 1$ H, H-6a), 3.15 (dd, $J=13.4,2.7 \mathrm{~Hz}, 1 \mathrm{H}, \mathrm{H}-6 \mathrm{~b}), 2.18-2.11$ (m, $\left.2 \mathrm{H}, \mathrm{C}(\mathrm{O}) \mathrm{CH}_{2}\right), 2.05-1.95$ $\left(\mathrm{m}, 12 \mathrm{H}, \mathrm{CH}=\mathrm{CHC}_{2}, 3 \times \mathrm{C}(\mathrm{O}) \mathrm{CH}_{3}\right), 1.64-1.53\left(\mathrm{~m}, 2 \mathrm{H}, \mathrm{C}(\mathrm{O}) \mathrm{CH}_{2} \mathrm{C}_{2}\right), 1.32-1.16(\mathrm{~m}, 42$ $\left.\mathrm{H}, 21 \times \mathrm{CH}_{2}\right), 0.86\left(\mathrm{t}, J=6.7 \mathrm{~Hz}, 6 \mathrm{H}, 2 \mathrm{x} \mathrm{CH}_{3}\right)$.

ESI-MS: $\quad$ calculated $\quad[\mathrm{M}+\mathrm{Na}]^{+}=949.6$ found $\quad[\mathrm{M}+\mathrm{Na}]^{+}=949.4$ 

glucopyranoside (3)

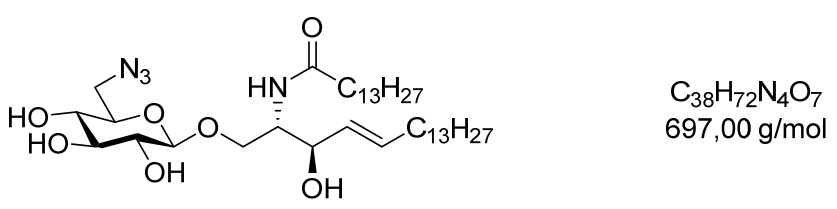

$\mathbf{3}$ was synthesized as described for $\mathbf{2}$, starting from $128 \mathrm{mg}(0.18 \mathrm{mmol}) \mathbf{3 5}$. After purification with silica chromatography (eluent DCM/MeOH 96:4) $61 \mathrm{mg} 3$ (0.09 mmol, $48 \%$ ) was obtained as colorless solid.

TLC: $R_{f}=0.26$ (eluent DCM/MeOH 9:1)

${ }^{1} \mathbf{H}$ NMR (400 MHz, $\left.\mathrm{CDCl}_{3}\right): \delta=6.74(\mathrm{~b}, 1 \mathrm{H}, \mathrm{NH}), 5.75(\mathrm{dt}, J=15.2 \mathrm{~Hz}, 7.2 \mathrm{~Hz}, 1 \mathrm{H}$, $\left.\mathrm{CH}=\mathrm{CHCH}_{2}\right), 5.44\left(\mathrm{dd}, J=15.3 \mathrm{~Hz}, 6.1 \mathrm{~Hz}, \underline{\mathrm{CH}}=\mathrm{CHCH}_{2}\right), 4.36(\mathrm{~d}, J=7.3 \mathrm{~Hz}, 1 \mathrm{H}, \mathrm{H}-1)$, $4.20-4.12$ (m, 2 H, $\underline{\mathrm{C}}(\mathrm{NHR}) \mathrm{C} \underline{\mathrm{H}}(\mathrm{OH}), 4.03-3.97$ (m, 1 H, $\left.\mathrm{CH}_{2} \mathrm{OGlc}\right), 3.78-3.72$ (m, $1 \mathrm{H}$, $\mathrm{CH}_{2} \mathrm{OGlc}$ ), $3.59-3.33$ (m, $\left.6 \mathrm{H}, \mathrm{H}-2, \mathrm{H}-3, \mathrm{H}-4, \mathrm{H}-5, \mathrm{H}-6 \mathrm{a} / \mathrm{b}\right), 2.25$ (t, $J=7.4 \mathrm{~Hz}, 2 \mathrm{H}$, $\left.\mathrm{C}(\mathrm{O}) \mathrm{CH}_{2}\right), 2.05-2.00\left(\mathrm{~m}, 2 \mathrm{H}, \mathrm{CH}=\mathrm{CHC}_{2}\right), 1.63-1.56\left(\mathrm{~m}, 2 \mathrm{H}, \mathrm{C}(\mathrm{O}) \mathrm{CH}_{2} \underline{\mathrm{C}}_{2}\right), 1.38-$ $1.17(\mathrm{~m}, 42 \mathrm{H}, 21 \mathrm{x} \mathrm{CH}), 0.88$ (t, $\left.J=6.8 \mathrm{~Hz}, 6 \mathrm{H}, 2 \mathrm{x} \mathrm{CH}_{3}\right)$.

${ }^{13} \mathrm{C}$ NMR $\left(101 \mathrm{MHz}, \mathrm{CDCl}_{3}\right): \delta=175.2(\mathrm{C}(\mathrm{O})), 135.1\left(\mathrm{CH}=\underline{\mathrm{CHCH}_{2}}\right), 128.2\left(\underline{\mathrm{C}} \mathrm{H}=\mathrm{CHCH}_{2}\right)$, 103.0 (C1), 76.4, 75.7, 73.5, 73.1, 71.2 (C2, C3, C4, C5, sphingosine-CHOH), 69.3 (sphingosine- $\left.\mathrm{CH}_{2} \mathrm{OH}\right), 53.7(\mathrm{CNH}), 51.7(\mathrm{C} 6), 36.9\left(\mathrm{C}(\mathrm{O}) \underline{\mathrm{CH}}_{2}\right), 32.5\left(\mathrm{CH}=\mathrm{CHCH}_{2}\right), 32.1$, $29.9-29.4(\mathrm{~m}), 26.0\left(\mathrm{CH}_{2}\right), 14.3\left(2 \mathrm{x} \mathrm{CH}_{3}\right)$.

ESI-MS: $\quad$ calculated $\quad[\mathrm{M}+\mathrm{H}]^{+}=697.5,[\mathrm{M}+\mathrm{Na}]^{+}=719.5$ found $\quad[\mathrm{M}+\mathrm{H}]^{+}=697.4,[\mathrm{M}+\mathrm{Na}]^{+}=719.2$

HR-ESI-MS: calculated $[\mathrm{M}+\mathrm{H}]^{+}=697.54738$ found $\quad[\mathrm{M}+\mathrm{H}]^{+}=697.54749$ 
Synthesis of Rhodamine Azide 5<smiles></smiles>

Rhodamine B-piperazinamide $^{1,2}(150 \mathrm{mg}, 0.32 \mathrm{mmol})$ was dissolved in $15 \mathrm{ml}$ dry DCM. After addition of azidoacetic acid $N$-succinimidyl ester ${ }^{3}(69 \mathrm{mg}, 0.35 \mathrm{mmol}$ ) and DIPEA (216 $\mu \mathrm{L}, 1.26 \mathrm{mmol}$ ), the reaction was stirred for 3 days at room temperature. The solvent was removed under reduced pressure. The crude product was purified by silica column chromatography (eluent DCM/MeOH 9:1), and 5 was obtained as a dark purple solid (95 mg, $0.16 \mathrm{mmol}, 50 \%)$.

TLC: $R_{f}=0.19$ (eluent DCM/MeOH 9:1)

${ }^{1} \mathbf{H}$ NMR (400 MHz, CD $\left.3 \mathrm{OD}\right): \delta=7.80-7.75$ (m, $2 \mathrm{H}$, aromat.), $7.73-7.69$ (m, $1 \mathrm{H}$, aromat.), $7.55-7.51$ (m, $1 \mathrm{H}$, aromat.), 7.28 (d, $J=9.5 \mathrm{~Hz}, 2 \mathrm{H}$, aromat.), 7.08 (dd, $J=9.6$, $2.5 \mathrm{~Hz}, 2 \mathrm{H}$, aromat.), 6.97 (d, $J=2.4 \mathrm{~Hz}, 2 \mathrm{H}$, aromat.), 3.69 (q, $J=7.1 \mathrm{~Hz}, 8 \mathrm{H}, 4 \mathrm{x}$ $\mathrm{CH}_{2} \mathrm{CH}_{3}$ ), 3.43 (br, $8 \mathrm{H}, 2 \mathrm{x} \mathrm{NCH} \mathrm{CH}_{2} \mathrm{~N}$ ), $3.30-3.26$ (obscured, $2 \mathrm{H}, \mathrm{CH}_{2} \mathrm{~N}_{3}$ ), 1.31 (t, $J=7.1$ $\left.\mathrm{Hz}, 12 \mathrm{H}, 4 \times \mathrm{CH}_{3}\right)$.

${ }^{13}$ C NMR (101 MHz, $\left.\mathrm{CD}_{3} \mathrm{OD}\right): \delta=159.3,157.2$ (2x C(O)), 157.0, 136.4, 133.24, 133.18, 132.4, 132.3, 131.8, 131.4, 129.9, 128.9, 115.4, 114.9, 97.4 (13x C aromat.), 49 (obscured, 2x $\left.\mathrm{NCH}_{2} \mathrm{CH}_{2} \mathrm{~N}\right), 46.9\left(4 \mathrm{x} \mathrm{N} \mathrm{NH}_{2} \mathrm{CH}_{3}\right), 45.2\left(\mathrm{CH}_{2} \mathrm{~N}_{3}\right), 12.8\left(\mathrm{CH}_{3}\right)$.

ESI-MS: $\quad$ calculated $\quad[\mathrm{M}]^{+}=594.3$

found $\quad[\mathrm{M}]^{+}=594.3$ 


\section{Synthesis of DIBO-Lissamine 6}

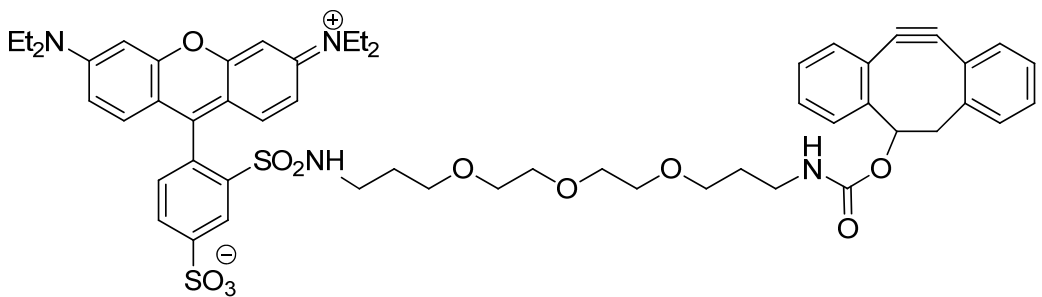

$\mathrm{C}_{54} \mathrm{H}_{62} \mathrm{~N}_{4} \mathrm{O}_{11} \mathrm{~S}_{2}$

Carbonic acid 7,8-didehydro-1,2:5,6-dibenzocyclooctene-3-yl ester 4-nitrophenyl ester ${ }^{4}$ (44 mg, $\quad 0.114 \quad \mathrm{mmol}), \quad N$-(1-amino-4,7,10-trioxa-tetradec-13-yl)-sulforhodamin-Bsulfonamide-2,2,2-trifluoroacetate $^{5}$ (100 mg, $0.114 \mathrm{mmol}$ ), and DIPEA (39 $\mu \mathrm{L}, 0.228 \mathrm{mmol}$, $30 \mathrm{mg}$ ) were dissolved in $10 \mathrm{~mL}$ dry DMF. The reaction was stirred for $4 \mathrm{~h}$ at room temperature, followed by evaporation at reduced pressure. After purification by silica column chromatography (eluent DCM/MeOH 95:5), 6 was obtained as a purple solid (80 mg, 80 $\mu \mathrm{mol}, 70 \%$, mixture of isomers).

TLC: $R_{f}=0.37$ (eluent DCM/MeOH 9:1)

${ }^{1} \mathbf{H}$ NMR (400 MHz, $\left.\mathrm{CDCl}_{3}\right): \delta=8.74-8.69$ (m, $1 \mathrm{H}$, aromat.), $8.00-7.96$ (m, $1 \mathrm{H}$, aromat.), $7.49-7.47$ (m, $1 \mathrm{H}$, aromat.), $7.34-7.14$ (m, $10 \mathrm{H}$, aromat.), $6.80-6.73(\mathrm{~m}, 2 \mathrm{H}$, aromat.), 6.66 - 6.63 (m, 2 H, aromat.), 5.41 (br, $1 \mathrm{H}$, CHO cyclooctyne), $3.72-3.47$ (m, 20 $\left.\mathrm{H}, 6 \mathrm{x} \mathrm{OCH}_{2}, 4 \mathrm{x} \mathrm{NC} \underline{H}_{2} \mathrm{CH}_{3}\right), 3.26-3.23\left(\mathrm{~m}, 2 \mathrm{H}, \mathrm{NCH}_{2}\right), 3.16-3.11\left(\mathrm{~m}, 3 \mathrm{H}, \mathrm{NCH}_{2}, \mathrm{CH}_{2}\right.$ cyclooctyne), 2.81 (dd, $J=14.9,3.6 \mathrm{~Hz}, 1 \mathrm{H}, \mathrm{CH}_{2}$ cyclooctyne), $1.84-1.73(\mathrm{~m}, 4 \mathrm{H}, 2 \mathrm{x}$ $\left.\mathrm{CH}_{2}\right), 1.28-1.23\left(\mathrm{~m}, 12 \mathrm{H}, \mathrm{NCH}_{2} \underline{\mathrm{C}}_{3}\right)$.

${ }^{13}$ C NMR (101 MHz, $\mathrm{CDCl}_{3}$ ): $\delta=158.4$ (C(O)), 158.0 (2x C aromat.), 155.6 (2x C aromat.), 152.5, 151.3, 147.6 (3x C aromat.), 142.4 (C aromat.), 133.6 (C aromat.), 133.3 (2x C aromat.), 130.1 (2x C aromat.), 130.0 (C aromat.), 128.1 (2x C aromat.), 127.5 (C aromat.), 127.0 (2x C aromat.), 126.1, 125.90, 124.1, 123.9, 121.2 (5x C aromat.), 114.4 (2x C aromat.), 113.7 (2x C aromat.), 112.8 (C aromat.), 110.1 (2x C Alkin), 95.7 (2x C aromat.), 76.5 (CHOR cyclooctyne), 70.7, 70.6, 70.24, 70.21, 69.5, $69.2\left(6 \mathrm{x} \mathrm{OCH}_{2}\right), 46.3\left(\mathrm{CH}_{2}\right.$ cyclooctyne), 45.9 (4x $\left.\mathrm{NCH}_{2} \mathrm{CH}_{3}\right), 41.4\left(\mathrm{NHCH}_{2}\right), 38.8\left(\mathrm{NHCH}_{2}\right), 29.5,29.3\left(2 \mathrm{x} \mathrm{CH}_{2}\right), 12.6$ $\left(4 \mathrm{x} \mathrm{NCH}_{2} \mathrm{CH}_{3}\right)$.

MALDI-MS: calculated $[\mathrm{M}+\mathrm{H}]^{+}=1007.4,[\mathrm{M}+\mathrm{Na}]^{+}=1029.4$

$$
\text { found } \quad[\mathrm{M}+\mathrm{H}]^{+}=1007.3,[\mathrm{M}+\mathrm{Na}]^{+}=1029.3
$$


Synthesis of Azidolactosylceramides 47, 48, and 49

$N$-((2S,3R,E)-3-Hydroxy-1-(trityloxy)octadec-4-en-2-yl)octanamide (20)<smiles>[CH]OCC(NC(C)=O)C(O)C=CCCCCCCO</smiles>

20 was prepared as described for 19, starting from $100 \mathrm{mg}(0.24 \mathrm{mmol})$ ceramide 16. After purification by silica chromatography (eluent petroleum ether/ethyl acetate 3:1) 20 was obtained as a colorless solid (76.2 $\mathrm{mg}, 0.114 \mathrm{mmol}, 49 \%)$.

TLC: $R_{f}=0.19$ (eluent petroleum ether/ethyl acetate 3:1)

${ }^{1} \mathbf{H}$ NMR (400 MHz, $\left.\mathrm{CDCl}_{3}\right): \delta=7.40-7.37$ (m, $6 \mathrm{H}$, aromat.), $7.31-7.20$ (m, $9 \mathrm{H}$, aromat.), 6.03 (d, $J=7.9 \mathrm{~Hz}, 1 \mathrm{H}, \mathrm{NH}), 5.66-5.59$ (m, $\left.1 \mathrm{H}, \mathrm{CH}=\mathrm{CHCH}_{2}\right), 5.28-5.22$ (m, 1 $\left.\mathrm{H}, \underline{\mathrm{C}} \underline{\mathrm{H}}=\mathrm{CHCH}_{2}\right), 4.18-4.15(\mathrm{~m}, 1 \mathrm{H}, \underline{\mathrm{C}} \underline{\mathrm{HOH}}), 4.07-4.01(\mathrm{~m}, 1 \mathrm{H}, \mathrm{C} \underline{\mathrm{HNH}}), 3.37$ (dd, $J=$ 9.7, $3.8 \mathrm{~Hz}, 1 \mathrm{H}, \mathrm{CH}_{2} \mathrm{OTrt}$ ), 3.29 (dd, $J=9.7,4.1 \mathrm{~Hz}, 1 \mathrm{H}, \mathrm{CH}_{2} \mathrm{OTrt}$ ), $2.22-2.15$ (m, $2 \mathrm{H}$, $\left.\mathrm{C}(\mathrm{O}) \mathrm{CH}_{2}\right), 1.95-1.85\left(\mathrm{~m}, 2 \mathrm{H}, \mathrm{CH}=\mathrm{CHC}_{2}\right), 1.67-1.57\left(\mathrm{~m}, 2 \mathrm{H}, \mathrm{C}(\mathrm{O}) \mathrm{CH}_{2} \underline{\mathrm{C}}_{2}\right), 1.34$ $1.19\left(\mathrm{~m}, 30 \mathrm{H}, 15 \mathrm{x} \mathrm{CH}_{2}\right), 0.87$ (t, $\left.J=6.8 \mathrm{~Hz}, 6 \mathrm{H}, 2 \mathrm{x} \mathrm{CH}_{3}\right)$.

${ }^{13}$ C NMR (101 MHz, $\left.\mathrm{CDCl}_{3}\right) \delta 173.5(\mathrm{C}(\mathrm{O})), 143.5$ (C aromat.), $133.6(\mathrm{CH}=\underline{\mathrm{CHCH}} 2), 128.6$ $\left(\underline{\mathrm{CH}}=\mathrm{CHCH}_{2}\right), 128.2,128.08,128.05,127.5,127.4,(5 \mathrm{x} \mathrm{C}$ aromat. $), 87.5\left(\mathrm{CPh}_{3}\right), 74.5(\mathrm{COH})$, 63.2 (COTrt), $53.6(\mathrm{CNH}), 37.0\left(\mathrm{C}(\mathrm{O}) \underline{\mathrm{CH}}_{2}\right), 32.1\left(\mathrm{CH}=\mathrm{CHCH}_{2}\right), 31.8\left(\mathrm{CH}_{2}\right), 29.8-29.2(\mathrm{~m}$, $\left.\mathrm{CH}_{2}\right), 22.8\left(\mathrm{CH}_{2}\right), 21.2\left(\mathrm{C}(\mathrm{O}) \mathrm{CH}_{2} \underline{\mathrm{CH}}_{2}\right), 14.24,14.19\left(2 \mathrm{x} \mathrm{CH}_{3}\right)$.

ESI-MS: $\quad$ calculated $\quad[\mathrm{M}+\mathrm{H}]^{+}=668.5,[\mathrm{M}+\mathrm{Na}]^{+}=690.5$ found $[\mathrm{M}+\mathrm{H}]^{+}=668.7,[\mathrm{M}+\mathrm{Na}]^{+}=690.7$

\section{$N-[(1 S, 2 R, 3 E)-2-H y d r o x y-1-[($ triphenylmethoxy)methyl]-3-heptadecen-1-yl]-} eicosanamide (22)

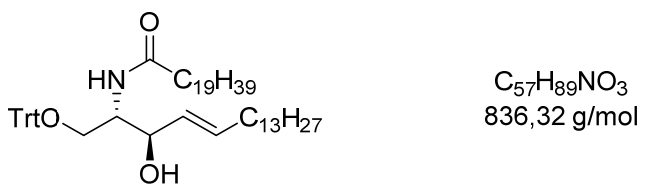

22 was synthesized as described for 19, starting from $200 \mathrm{mg}(0.34 \mathrm{mmol})$ ceramide 18. After purification with silica chromatography (eluent petroleum ether/ethyl acetate 3:1) $273 \mathrm{mg} 22$ (0.33 mmol, quant.) was obtained as a colorless solid.

TLC: $R_{f}=0.20$ (eluent petroleum ether/ethyl acetate $3: 1$ )

${ }^{1} \mathbf{H}$ NMR (400 MHz, $\left.\mathrm{CDCl}_{3}\right): \delta=7.39-7.36$ (m, $6 \mathrm{H}$, aromat.), $7.30-7.20$ (m, $9 \mathrm{H}$, aromat.), 6.06 (d, $J=8.0 \mathrm{~Hz}, 1 \mathrm{H}, \mathrm{NH}), 5.65-5.58$ (m, $\left.1 \mathrm{H}, \mathrm{CH}=\mathrm{CHCH}_{2}\right), 5.27-5.21$ (m, 1 $\left.\mathrm{H}, \mathrm{C} \underline{\mathrm{H}}=\mathrm{CHCH}_{2}\right), 4.18-4.14(\mathrm{~m}, 1 \mathrm{H}, \mathrm{C} \underline{\mathrm{HOH}}), 4.06-4.01(\mathrm{~m}, 1 \mathrm{H}, \mathrm{C} \underline{\mathrm{HNH}}), 3.36(\mathrm{dd}, J=$ 
9.7, $\left.3.8 \mathrm{~Hz}, 1 \mathrm{H}, \mathrm{CH}_{2} \mathrm{OTrt}\right), 3.28$ (dd, $J=9.7,4.1 \mathrm{~Hz}, 1 \mathrm{H}, \mathrm{CH}_{2} \mathrm{OTrt}$ ), $2.20-2.14$ (m, $2 \mathrm{H}$, $\left.\mathrm{C}(\mathrm{O}) \mathrm{CH}_{2}\right), 1.94-1.86\left(\mathrm{~m}, 2 \mathrm{H}, \mathrm{CH}=\mathrm{CHC}_{2}\right), 1.65-1.58\left(\mathrm{~m}, 2 \mathrm{H}, \mathrm{C}(\mathrm{O}) \mathrm{CH}_{2} \underline{\mathrm{C}}_{2}\right), 1.34$ $1.17\left(\mathrm{~m}, 54 \mathrm{H}, 27 \mathrm{x} \mathrm{CH}_{2}\right), 0.86$ (t, $\left.J=6.8 \mathrm{~Hz}, 6 \mathrm{H}, 2 \mathrm{x} \mathrm{CH}_{3}\right)$.

${ }^{13} \mathrm{C}$ NMR (101 MHz, $\left.\mathrm{CDCl}_{3}\right): \delta=173.5(\mathrm{C}(\mathrm{O})), 143.5$ (C aromat.), $133.5\left(\mathrm{CH}=\underline{\mathrm{CHCH}_{2}}\right)$, $128.6\left(\underline{\mathrm{CH}}=\mathrm{CHCH}_{2}\right), 128.12,128.06,128.00,127.4,127.3$ (5x C aromat.), $87.5\left(\mathrm{CPh}_{3}\right), 74.4$ $(\mathrm{COH}), 63.2\left(\mathrm{CH}_{2} \mathrm{OTrt}\right), 53.5(\mathrm{CHNH}), 37.0\left(\mathrm{C}(\mathrm{O}) \underline{\mathrm{CH}_{2}}\right), 32.1\left(\mathrm{CH}=\mathrm{CHCH}_{2}\right), 29.8$ - 29.2, 26.0, 22.9, $21.1\left(\mathrm{CH}_{2}\right), 14.3,14.2\left(2 \mathrm{x} \mathrm{CH}_{3}\right)$.

ESI-MS: $\quad$ calculated $\quad[\mathrm{M}+\mathrm{Na}]^{+}=858.7,[\mathrm{M}-\mathrm{H}]^{-}=834.7$

found $\quad[\mathrm{M}+\mathrm{Na}]^{+}=858.3,[\mathrm{M}-\mathrm{H}]^{-}=834.2$

(2S,3R,E)-2-Octanamido-1-(trityloxy)octadec-4-en-3-yl benzoate (24)

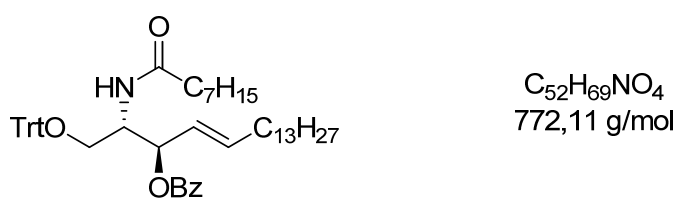

24 was prepared as described for 23, starting from $76 \mathrm{mg}(0.114 \mathrm{mmol})$ ceramide 20. After purification by silica chromatography (eluent petroleum ether/ethyl acetate 7:1) 24 was obtained as a colorless solid (48 $\mathrm{mg}, 0.063 \mathrm{mmol}, 55 \%$ ).

TLC: $R_{f}=0.44$ (eluent petroleum ether/ethyl acetate $4: 1$ )

${ }^{1} \mathbf{H}$ NMR (400 MHz, $\left.\mathrm{CDCl}_{3}\right): \delta=8.14-8.12$ (m, $2 \mathrm{H}$, aromat.), $7.92-7.90$ (m, $2 \mathrm{H}$, aromat.), $7.66-7.62$ (m, $1 \mathrm{H}$, aromat.), $7.57-7.12$ (m, $15 \mathrm{H}$, aromat.), 5.85 (dt, $J=13.6,6.8$ $\left.\mathrm{Hz}, 1 \mathrm{H}, \mathrm{CH}=\mathrm{CHCH}_{2}\right), 5.70-5.63(\mathrm{~m}, 2 \mathrm{H}, \mathrm{NH}, \mathrm{CHOBz}), 5.42$ (dd, $J=15.4,7.5 \mathrm{~Hz}, 1 \mathrm{H}$, $\left.\mathrm{C} \underline{\mathrm{H}}=\mathrm{CHCH}_{2}\right), 4.49-4.43(\mathrm{~m}, 1 \mathrm{H}, \mathrm{C} \underline{\mathrm{HNH}}), 3.42\left(\mathrm{dd}, J=9.5,3.6 \mathrm{~Hz}, 1 \mathrm{H}, \mathrm{CH}_{2} \mathrm{OTrt}\right), 3.17$ (dd, $\left.J=9.5,4.1 \mathrm{~Hz}, 1 \mathrm{H}, \mathrm{CH}_{2} \mathrm{OTrt}\right), 2.07$ (t, $\left.J=7.6 \mathrm{~Hz}, 2 \mathrm{H}, \mathrm{C}(\mathrm{O}) \mathrm{CH}_{2}\right), 2.01-1.94$ (m, $2 \mathrm{H}$, $\left.\mathrm{CH}=\mathrm{CHC}_{2}\right), 1.60-1.51\left(\mathrm{~m}, 2 \mathrm{H}, \mathrm{C}(\mathrm{O}) \mathrm{CH}_{2} \mathrm{C}_{2}\right), 1.33-1.14\left(\mathrm{~m}, 30 \mathrm{H}, 15 \mathrm{x} \mathrm{CH}_{2}\right), 0.87-$ $0.83\left(\mathrm{~m}, 6 \mathrm{H}, 2 \mathrm{x} \mathrm{CH}_{3}\right)$.

${ }^{13}$ C NMR (101 MHz, $\left.\mathrm{CDCl}_{3}\right): \delta=172.6,165.5$ (2x C(O)), 147.0, 143.6 (2x C aromat.), 137.3 $\left(\mathrm{CH}=\underline{\mathrm{C} H C H}{ }_{2}\right), 134.7,133.0,130.7,129.0,128.7,128.4,128.06,128.03,128.0,127.4(10 \mathrm{x} \mathrm{C}$ aromat.), $125.2\left(\underline{\mathrm{CH}}=\mathrm{CHCH}_{2}\right), 87.0\left(\mathrm{CPh}_{3}\right), 74.5$ (COBz), 61.8 (COTrt), $51.2(\mathrm{CNH}), 37.1$ $\left(\mathrm{C}(\mathrm{O}) \underline{\mathrm{CH}}_{2}\right), 32.5\left(\mathrm{CH}=\mathrm{CH} \underline{C H}_{2}\right), 32.1,31.8,29.8$ - 29.0 (m), 25.9, 22.8, $22.8\left(\mathrm{CH}_{2}\right), 14.24$, $14.19\left(2 \mathrm{X} \mathrm{CH}_{3}\right)$. 


\section{$N-[(1 S, 2 R, 3 E)-2-(B e n z o y l o x y)-1-[(t r i p h e n y l m e t h o x y) m e t h y l]-3-h e p t a d e c e n-1-y l]-$}

eicosanamide (26)

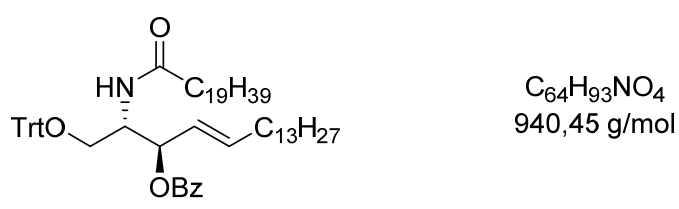

26 was synthesized as described for $\mathbf{2 4}$, starting from $270 \mathrm{mg}(0.33 \mathrm{mmol})$ ceramide $\mathbf{2 2}$. After purification with silica chromatography (eluent petroleum ether/ethyl acetate 7:1) $144 \mathrm{mg} 26$ ( $0.153 \mathrm{mmol}, 46 \%)$ was obtained as colorless solid.

TLC: $R_{f}=0.43$ (eluent petroleum ether/ethyl acetate 4:1)

${ }^{1} \mathbf{H}$ NMR (400 MHz, $\left.\mathrm{CDCl}_{3}\right): \delta=8.15-8.12$ (m, $2 \mathrm{H}$, aromat.), $7.93-7.90$ (m, $2 \mathrm{H}$, aromat.), $7.67-7.62$ (m, $2 \mathrm{H}$, aromat.), $7.55-7.48$ (m, $6 \mathrm{H}$, aromat.) $7.40-7.12$ (m, $8 \mathrm{H}$, aromat.), $5.89-5.82$ (dt, $J=15.8,6.8 \mathrm{~Hz}, 1 \mathrm{H}, \mathrm{CH}=\mathrm{CHCH}_{2}$ ), $5.70-5.63$ (m, $2 \mathrm{H}, \mathrm{CHOBz}$, $\mathrm{NH}), 5.46-5.40$ (m, $\left.1 \mathrm{H}, \mathrm{C} \underline{\mathrm{H}}=\mathrm{CHCH}_{2}\right), 4.50-4.43$ (m, $\left.1 \mathrm{H}, \mathrm{C} \underline{\mathrm{HNH}}\right), 3.42$ (dd, $J=9.5,3.6$ $\mathrm{Hz}, 1 \mathrm{H}, \mathrm{CH}_{2} \mathrm{OTrt}$ ), 3.17 (dd, $J=9.5,4.2 \mathrm{~Hz}, 1 \mathrm{H}, \mathrm{CH}_{2} \mathrm{OTrt}$ ), 2.07 (t, $J=7.6 \mathrm{~Hz}, 2 \mathrm{H}$, $\left.\mathrm{C}(\mathrm{O}) \mathrm{CH}_{2}\right), 2.00-1.94\left(\mathrm{~m}, 2 \mathrm{H}, \mathrm{CH}=\mathrm{CHC}_{2}\right), 1.59-1.51\left(\mathrm{~m}, 2 \mathrm{H}, \mathrm{C}(\mathrm{O}) \mathrm{CH}_{2} \underline{\mathrm{C}}_{2}\right), 1.36$ $1.16\left(\mathrm{~m}, 54 \mathrm{H}, 27 \mathrm{x} \mathrm{CH}\right.$ ), 0.86 (t, $\left.J=6.8 \mathrm{~Hz}, 6 \mathrm{H}, 2 \mathrm{x} \mathrm{CH}_{3}\right)$.

${ }^{13}$ C NMR (101 MHz, $\left.\mathrm{CDCl}_{3}\right): \delta=172.6,162.5$ (2x C(O)), 147.0, 143.6 (2x C aromat.), 137.3 $\left(\mathrm{CH}=\underline{\mathrm{C}} \mathrm{HCH}_{2}\right), 134.7,130.7,129.9,129.0,128.7,128.1,128.04,127.98,127.4,127.2$ (10x C aromat.), $125.3\left(\underline{\mathrm{CH}}=\mathrm{CHCH}_{2}\right), 87.0\left(\mathrm{CPh}_{3}\right), 74.6$ (COBz), 61.8 (COTrt), $51.2(\mathrm{CNH}), 37.1$ $\left(\mathrm{C}(\mathrm{O}) \underline{\mathrm{C}} \mathrm{H}_{2}\right), 32.5\left(\mathrm{CH}=\mathrm{CH} \mathrm{H}_{2}\right), 32.1,29.9-29.4(\mathrm{~m}), 29.1,26.0,22.8\left(\mathrm{CH}_{2}\right), 14.3\left(2 \times \mathrm{CH}_{3}\right)$.

ESI-MS: $\quad$ calculated $[\mathrm{M}+\mathrm{Na}]^{+}=962.7$ found $\quad[\mathrm{M}+\mathrm{Na}]^{+}=962.1$

\section{(2S,3R,E)-2-Octanamido-1-hydroxyoctadec-4-en-3-yl benzoate (28)}

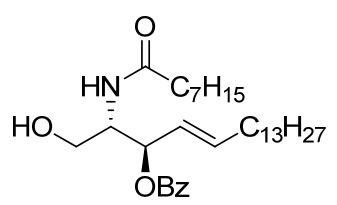

28 was prepared as described for $\mathbf{2 7}$, starting from $118 \mathrm{mg}(0.153 \mathrm{mmol})$ ceramide $\mathbf{2 4}$. After purification by silica chromatography (eluent petroleum ether/ethyl acetate 1:1, then 2:3) 28 was obtained as a colorless solid (64 $\mathrm{mg}, 0.120 \mathrm{mmol}, 78 \%$ ).

TLC: $R_{f}=0.18$ (eluent petroleum ether/ethyl acetate 1:1)

${ }^{1} \mathbf{H}$ NMR (400 MHz, $\left.\mathrm{CDCl}_{3}\right): \delta=8.05-8.02$ (m, $2 \mathrm{H}$, aromat.), $7.61-7.57$ (m, $1 \mathrm{H}$, aromat.), $7.47-7.44$ (m, $2 \mathrm{H}$, aromat.), 6.07 (d, $J=8.6 \mathrm{~Hz}, 1 \mathrm{H}, \mathrm{NH}), 5.89-5.82$ (dt, $J=$ 14.8, $\left.6.8 \mathrm{~Hz}, 1 \mathrm{H}, \mathrm{CH}=\mathrm{CHCH}_{2}\right), 5.63-5.52$ (m, $\left.2 \mathrm{H}, \mathrm{C} \underline{\mathrm{H}}=\mathrm{CHCH}_{2}, \mathrm{CHOBz}\right), 4.27$ (ddt, $J=$ 
8.6, 7.0, $3.5 \mathrm{~Hz}, 1 \mathrm{H}, \mathrm{C} \underline{\mathrm{HNH}}), 3.75\left(\mathrm{dd}, J=11.9,3.8 \mathrm{~Hz}, 1 \mathrm{H}, \underline{\mathrm{C}}_{2} \mathrm{OH}\right), 3.70$ (dd, $J=12.0$, $\left.3.2 \mathrm{~Hz}, 1 \mathrm{H}, \underline{\mathrm{C}}_{2} \mathrm{OH}\right), 2.22-2.17\left(\mathrm{~m}, 2 \mathrm{H}, \mathrm{C}(\mathrm{O}) \mathrm{CH}_{2}\right), 2.07-2.02\left(\mathrm{~m}, 2 \mathrm{H}, \mathrm{CH}=\mathrm{CHCH}_{2}\right)$, $1.65-1.58\left(\mathrm{~m}, 2 \mathrm{H}, \mathrm{C}(\mathrm{O}) \mathrm{CH}_{2} \mathrm{C}_{2}\right), 1.39-1.20\left(\mathrm{~m}, 30 \mathrm{H}, 15 \mathrm{x} \mathrm{CH}_{2}\right), 0.88(\mathrm{t}, J=6.9 \mathrm{~Hz}, 3 \mathrm{H}$, $\left.\mathrm{CH}_{3}\right), 0.87\left(\mathrm{t}, J=6.9 \mathrm{~Hz}, 3 \mathrm{H}, \mathrm{CH}_{3}\right)$.

${ }^{13} \mathbf{C}$ NMR (101 MHz, $\left.\mathrm{CDCl}_{3}\right): \delta=173.6,166.7(2 \mathrm{x} \mathrm{C}(\mathrm{O})), 137.7\left(\mathrm{CH}=\underline{\mathrm{C} H C H}{ }_{2}\right), 133.6(\mathrm{C}$ aromat.), 130.0 (2x C aromat.), 129.8 (C aromat.), 128.7 (2x C aromat), $125.0\left(\underline{\mathrm{CH}}=\mathrm{CHCH}_{2}\right)$, $74.9(\mathrm{CHOBz}), 62.1\left(\mathrm{CH}_{2} \mathrm{OH}\right), 53.7(\mathrm{CHNH}), 37.0\left(\mathrm{C}(\mathrm{O}) \underline{\mathrm{CH}}_{2}\right), 32.5\left(\mathrm{CH}=\mathrm{CHCH}_{2}\right), 32.1$, 31.8, 29.83 - 29.80 (m), 29.6, 29.5, 29.4, 29.2, 29.1, 25.9, $22.8\left(\mathrm{CH}_{2}\right), 14.24,14.18\left(2 \mathrm{x} \mathrm{CH}_{3}\right)$.

ESI-MS: $\quad$ calculated $\quad[\mathrm{M}+\mathrm{H}]^{+}=530.4,[\mathrm{M}+\mathrm{Na}]^{+}=552.4$ found $\quad[\mathrm{M}+\mathrm{H}]^{+}=530.4,[\mathrm{M}+\mathrm{Na}]^{+}=552.4$

$N$-[(1S,2R,3E)-2-(Benzoyloxy)-1-(hydroxymethyl)-3-heptadecen-1-yl]-eicosanamide (30)

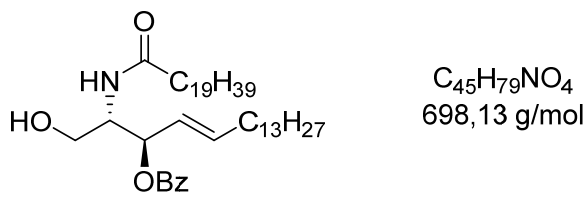

30 was synthesized as described for 27, starting from $140 \mathrm{mg} 26$ (0.153 mmol). After purification with silica chromatography (eluent petroleum ether/ethyl acetate 2:1) $58 \mathrm{mg} 30$ $(0.083 \mathrm{mmol}, 54 \%)$ was obtained as a colorless solid.

TLC: $R_{f}=0.46$ (eluent petroleum ether/ethyl acetate 1:1)

${ }^{1} \mathbf{H}$ NMR (400 MHz, $\mathrm{CDCl}_{3}$ ): $\delta=8.03$ (d, $J=7.5 \mathrm{~Hz}, 2 \mathrm{H}$, aromat.), 7.58 (t, $J=7.4 \mathrm{~Hz}, 1 \mathrm{H}$, aromat.), 7.45 (t, $J=7.7 \mathrm{~Hz}, 2 \mathrm{H}$, aromat.), 6.11 (d, $J=8.7 \mathrm{~Hz}, 1 \mathrm{H}, \mathrm{NH}), 5.89-5.82$ (dt, $J=$ 14.9, $\left.6.7 \mathrm{~Hz}, 1 \mathrm{H}, \mathrm{CH}=\mathrm{CHCH}_{2}\right), 5.63-5.52\left(\mathrm{~m}, 2 \mathrm{H}, \mathrm{CH}=\mathrm{CHCH}_{2}, \mathrm{CHOBz}\right), 4.31-4.25$ (m, $1 \mathrm{H}, \mathrm{C} \underline{\mathrm{H} N H}), 3.76-3.67\left(\mathrm{~m}, 2 \mathrm{H}, \underline{\mathrm{C}}_{2} \mathrm{OH}\right), 3.02(\mathrm{~b}, 1 \mathrm{H}, \mathrm{OH}), 2.24-2.12(\mathrm{~m}, 2 \mathrm{H}$, $\left.\mathrm{C}(\mathrm{O}) \mathrm{CH}_{2}\right), 2.07-2.01\left(\mathrm{~m}, 2 \mathrm{H}, \mathrm{CH}=\mathrm{CHCH}_{2}\right), 1.64-1.57\left(\mathrm{~m}, 2 \mathrm{H}, \mathrm{C}(\mathrm{O}) \mathrm{CH}_{2} \underline{\mathrm{C}}_{2}\right), 1.40-$ $1.13(\mathrm{~m}, 54 \mathrm{H}, 27 \mathrm{x} \mathrm{CH}), 0.87$ (t, $\left.J=6.7 \mathrm{~Hz}, 6 \mathrm{H}, 2 \mathrm{x} \mathrm{CH}_{3}\right)$.

${ }^{13} \mathrm{C}$ NMR $\left(101 \mathrm{MHz}, \mathrm{CDCl}_{3}\right): \delta=173.5,166.6(2 \mathrm{x} \mathrm{C}(\mathrm{O})), 137.6\left(\mathrm{CH}=\underline{\mathrm{CHCH}_{2}}\right), 133.6(\mathrm{C}$ aromat.), 130.0 (2x C aromat.), 129.8 (C aromat.), 128.6 (2x C aromat.), 125.0 ( $\underline{\mathrm{CH}}=\mathrm{CHCH}_{2}$ ), $74.8(\mathrm{COBz}), 62.0(\mathrm{COH}), 53.6(\mathrm{CNH}), 37.0\left(\mathrm{C}(\mathrm{O}) \underline{\mathrm{CH}}_{2}\right), 32.5\left(\mathrm{CH}=\mathrm{CHCH}_{2}\right), 32.1,29.8-$ 29.5 (m), 29.4, 29.1, 26.0, $22.9\left(\mathrm{CH}_{2}\right), 14.3\left(2 \mathrm{x} \mathrm{CH}_{3}\right)$.

ESI-MS: $\quad$ calculated $\quad[\mathrm{M}+\mathrm{Na}]^{+}=720.6$ found $\quad[\mathrm{M}+\mathrm{Na}]^{+}=720.3$ 
(2S,3S,E)-2-Butyramido-3-(benzoyloxy)-octadec-4-en-1-yl $\quad 2,3,4-t r i-O$-acetyl-6-azido-6deoxy- $\beta$-D-galactopyranosyl-( $1 \rightarrow 4)-2,3,6$-tri- $O$-acetyl- $\beta$-D-glucopyranoside (44)

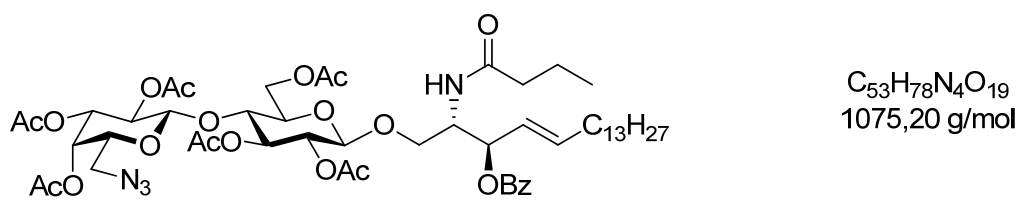

Azidolactosylceramide 44 was prepared as described for compound 34, starting from ceramide 27 (71 mg, $0.15 \mathrm{mmol})$ and trichloroacetimidate 43 (114 mg, $0.15 \mathrm{mmol})$. After silica column chromatography (eluent toluene/acetone 4:1) 44 was obtained as a colorless solid (66 mg, $62 \mu \mathrm{mol}, 41 \%$ ).

TLC: $R_{f}=0.45$ (eluent toluene/acetone 7:3)

${ }^{1} \mathbf{H}$ NMR (400 MHz, $\left.\mathrm{CDCl}_{3}\right): \delta=8.01-7.99$ (m, $2 \mathrm{H}$, aromat.), $7.57-7.53$ (m, $1 \mathrm{H}$, aromat.), $7.45-7.40$ (m, $2 \mathrm{H}$, aromat.), $5.89-5.82$ (m, $2 \mathrm{H}, \mathrm{NH}, \mathrm{CH}=\mathrm{CHCH}_{2}$ ), 5.53 (' $\mathrm{t}$ ', $J=$ $7.3 \mathrm{~Hz}, 1 \mathrm{H}, \mathrm{CHOBz}), 5.45$ (dd, $\left.J=15.3,7.5 \mathrm{~Hz}, 1 \mathrm{H}, \mathrm{C} \underline{\mathrm{H}}=\mathrm{CHCH}_{2}\right), 5.34-5.31$ (m, $1 \mathrm{H}, \mathrm{H}-$ 4'), 5.17 ('t', $J=9.3 \mathrm{~Hz}, 1 \mathrm{H}, \mathrm{H}-3$ ), 5.06 (dd, $J=10.3,7.9 \mathrm{~Hz}, 1 \mathrm{H}, \mathrm{H}-2$ '), 4.95 (dd, $J=10.3$, 3.5 Hz, 1 H, H-3'), 4.87 (dd, $J=9.3,8.0$ Hz, 1 H, H-2), 4.49 - 4.44 (m, 3 H, H-1, H-1', C $\underline{H N H}$ ), 4.35 (dd, $J=11.8,1.6$ Hz, $1 \mathrm{H}, \mathrm{H}-6 \mathrm{a}), 4.00$ - 3.95 (m, $\left.2 \mathrm{H}, \mathrm{H}-6 \mathrm{~b}, \mathrm{CH}_{2} \mathrm{OLac}\right), 3.82$ ('t', $J=9.5 \mathrm{~Hz}, 1 \mathrm{H}, \mathrm{H}-4), 3.73$ ('t', $J=6.5 \mathrm{~Hz}, 1 \mathrm{H}, \mathrm{H}-5$ '), 3.63 (dd, $J=10.1,4.3 \mathrm{~Hz}, 1 \mathrm{H}$, $\mathrm{CH}_{2} \mathrm{OLac}$ ), 3.59 - 3.54 (m, $\left.1 \mathrm{H}, \mathrm{H}-5\right), 3.44$ (dd, $J=12.8,7.3 \mathrm{~Hz}, 1 \mathrm{H}, \mathrm{H}-6 \mathrm{a}$ '), 3.26 - 3.21 (m, $1 \mathrm{H}, \mathrm{H}-6 \mathrm{~b}$ '), $2.16-2.12\left(\mathrm{~m}, 5 \mathrm{H}, \mathrm{C}(\mathrm{O}) \mathrm{CH}_{3}, \mathrm{C}(\mathrm{O}) \mathrm{CH}_{2}\right), 2.06$ (s, $\left.3 \mathrm{H}, \mathrm{C}(\mathrm{O}) \mathrm{CH}_{3}\right), 2.01$ (s, $3 \mathrm{H}$, $\left.\mathrm{C}(\mathrm{O}) \mathrm{CH}_{3}\right), 2.00\left(\mathrm{~s}, 3 \mathrm{H}, \mathrm{C}(\mathrm{O}) \mathrm{CH}_{3}\right), 1.95\left(\mathrm{~s}, 3 \mathrm{H}, \mathrm{C}(\mathrm{O}) \mathrm{CH}_{3}\right), 1.92\left(\mathrm{~s}, 3 \mathrm{H}, \mathrm{C}(\mathrm{O}) \mathrm{CH}_{3}\right), 1.65-$ $1.59\left(\mathrm{~m}, 2 \mathrm{H}, \mathrm{CH}=\mathrm{CHC}_{2}\right), 1.34-1.18\left(\mathrm{~m}, 24 \mathrm{H}, 12 \mathrm{x} \mathrm{CH}_{2}\right), 0.92\left(\mathrm{t}, J=7.4 \mathrm{~Hz}, 3 \mathrm{H}, \mathrm{CH}_{3}\right)$, $0.87\left(\mathrm{t}, J=6.9 \mathrm{~Hz}, 3 \mathrm{H}, \mathrm{CH}_{3}\right)$.

${ }^{13}$ C NMR (101 MHz, $\left.\mathrm{CDCl}_{3}\right): \delta=173.0(\mathrm{C}(\mathrm{O}) \mathrm{NH}), 170.5,170.21,170.15,169.9,169.8$, $169.3\left(6 \mathrm{x} \underline{\mathrm{C}}(\mathrm{O}) \mathrm{CH}_{3}\right), 165.4(\mathrm{C}(\mathrm{O}) \mathrm{Ph}), 136.0\left(\mathrm{CH}=\underline{\mathrm{CHCH}_{2}}\right), 129.7,129.0(2 \mathrm{x}), 128.7(2 \mathrm{x})$, 128.6 (C aromat.), $124.7\left(\underline{\mathrm{CH}}=\mathrm{CHCH}_{2}\right), 100.6(\mathrm{C} 1), 100.5$ (C1'), 75.4 (C4), 74.2 (CHOBz), 72.9 (C5), 72.6 (C3), 72.3 (C5'), 71.8 (C2), 71.0 (C3'), 69.2 (C2'), 67.6 (C4'), 67.5 ( $\left.\mathrm{CH}_{2} \mathrm{OLac}\right), 62.0$ (C6), $50.9(\mathrm{CHNH}), 50.3$ (C6'), 32.4, 32.0, 29.8 - 29.7 (m), 29.7, 29.68, 29.55, 29.4, 29.0, $22.8\left(\mathrm{CH}_{2}\right), 21.1,21.0,20.8(\mathrm{~m}), 20.7\left(\mathrm{C}(\mathrm{O}) \underline{\mathrm{CH}_{3}}\right), 19.2\left(\mathrm{CH}_{2}\right), 14.2,13.8$ $\left(2 \mathrm{x} \mathrm{CH}_{3}\right)$.

ESI-MS: $\quad$ calculated $\quad[\mathrm{M}+\mathrm{Na}]^{+}=1097.5$

found $\quad[\mathrm{M}+\mathrm{Na}]^{+}=1097.8$ 
(2S,3S,E)-2-Octanamido-3-(benzoyloxy)-octadec-4-en-1-yl $\quad 2,3,4-t r i-O$-acetyl-6-azido-6deoxy- $\beta$-D-galactopyranosyl-( $1 \rightarrow 4)-2,3,6$-tri- $O$-acetyl- $\beta$-D-glucopyranoside (45)

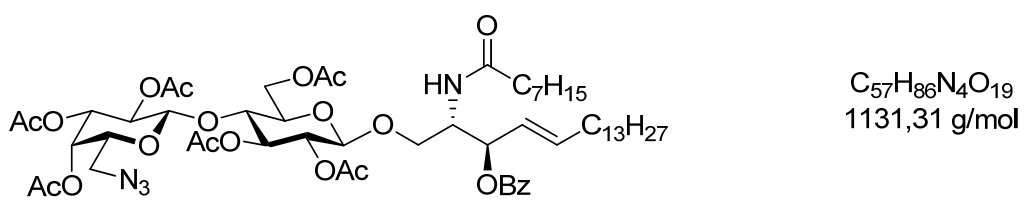

Azidolactosylceramide 45 was prepared as described for compound 34, starting from ceramide 28 (63 mg, $0.12 \mathrm{mmol})$ and trichloroacetimidate 43 (100 mg, $0.13 \mathrm{mmol})$. After silica column chromatography (eluent toluene/acetone 4:1) lactosylceramide $\mathbf{4 5}$ was obtained as a colorless solid (91 mg, $0.08 \mathrm{mmol}, 67 \%$ ).

TLC: $R_{f}=0.56$ (eluent toluene/acetone 7:3)

${ }^{1} \mathbf{H}$ NMR (400 MHz, $\left.\mathrm{CDCl}_{3}\right): \delta=8.02-7.99$ (m, $2 \mathrm{H}$, aromat.), $7.58-7.53$ (m, $1 \mathrm{H}$, aromat.), $7.45-7.41$ (m, $2 \mathrm{H}$, aromat.), 5.86 (dt, $J=14.7,6.7 \mathrm{~Hz}, 1 \mathrm{H}, \mathrm{CH}=\mathrm{C}_{\mathbf{H C H}}$ ), 5.74 (d, $J=9.2 \mathrm{~Hz}, 1 \mathrm{H}, \mathrm{NH}), 5.55-5.51(\mathrm{~m}, 1 \mathrm{H}, \mathrm{CHOBz}), 5.49-5.43\left(\mathrm{~m}, 1 \mathrm{H}, \mathrm{C} \underline{H}=\mathrm{CHCH}_{2}\right), 5.34$ (dd, $J=3.3,0.7 \mathrm{~Hz}, 1 \mathrm{H}, \mathrm{H}-4$ '), 5.18 ('t', $J=9.3 \mathrm{~Hz}, 1 \mathrm{H}, \mathrm{H}-3$ ), 5.07 (dd, $J=10.3,7.8 \mathrm{~Hz}, 1$ H, H-2'), 4.95 (dd, $J=10.4,3.4$ Hz, 1 H, H-3'), 4.88 (dd, $J=9.4,7.8$ Hz, 1 H, H-2), 4.48 (d, $J$ $\left.=7.8 \mathrm{~Hz}, 1 \mathrm{H}, \mathrm{H}-1^{\prime}\right), 4.49-4.43$ (m, 1 H, CㅌNH), 4.44 (d, J=7.7 Hz, $\left.1 \mathrm{H}, \mathrm{H}-1\right), 4.38$ (dd, $J$ $=11.9,1.9 \mathrm{~Hz}, 1 \mathrm{H}, \mathrm{H}-6 \mathrm{a}), 4.01-3.95$ (m, $\left.2 \mathrm{H}, \mathrm{H}-6 \mathrm{~b}, \mathrm{CH}_{2} \mathrm{OLac}\right), 3.82$ ('t', $J=9.5 \mathrm{~Hz}, 1 \mathrm{H}$, H-4), $3.73-3.70$ (m, 1 H, H-5'), 3.63 (dd, $J=10.1,4.5$ Hz, $1 \mathrm{H}, \mathrm{CH}_{2} \mathrm{OLac}$ ), 3.57 (ddd, $J=$ 9.8, 4.9, 2.0 Hz, 1 H, H-5), 3.48 - 3.43 (m, 1 H, H-6a'), 3.25 (dd, J=12.8, 5.7 Hz, 1 H, H6b'), $2.17-2.11\left(\mathrm{~m}, 5 \mathrm{H}, \mathrm{C}(\mathrm{O}) \mathrm{CH}_{3}, \mathrm{C}(\mathrm{O}) \mathrm{CH}_{2}\right), 2.06\left(\mathrm{~s}, 3 \mathrm{H}, \mathrm{C}(\mathrm{O}) \mathrm{CH}_{3}\right), 2.01(2 \mathrm{~s}, 6 \mathrm{H}, 2 \mathrm{x}$ $\left.\mathrm{C}(\mathrm{O}) \mathrm{CH}_{3}\right), 1.96\left(\mathrm{~s}, 3 \mathrm{H}, \mathrm{C}(\mathrm{O}) \mathrm{CH}_{3}\right), 1.95\left(\mathrm{~s}, 3 \mathrm{H}, \mathrm{C}(\mathrm{O}) \mathrm{CH}_{3}\right), 1.63-1.56(\mathrm{~m}, 2 \mathrm{H}$, $\left.\mathrm{CH}=\mathrm{CHC}_{2}\right), 1.37-1.20\left(\mathrm{~m}, 32 \mathrm{H}, 16 \mathrm{x} \mathrm{CH}_{2}\right), 0.87\left(\mathrm{t}, J=6.9 \mathrm{~Hz}, 3 \mathrm{H}, \mathrm{CH}_{3}\right), 0.86(\mathrm{t}, J=6.9$ $\mathrm{Hz}, 3 \mathrm{H}, \mathrm{CH}_{3}$ ).

${ }^{13}$ C NMR (101 MHz, $\left.\mathrm{CDCl}_{3}\right): \delta=172.8(\mathrm{C}(\mathrm{O}) \mathrm{NH}), 170.4,170.2,170.1,169.8,169.7,169.2$ (6x $\left.\underline{\mathrm{C}}(\mathrm{O}) \mathrm{CH}_{3}\right), 165.4(\mathrm{C}(\mathrm{O}) \mathrm{Ph}), 137.7\left(\mathrm{CH}=\underline{\mathrm{CHCH}}{ }_{2}\right), 133.2,130.4$ (2x C aromat.), 129.8, 128.6 (4x C aromat.), $124.8\left(\underline{\mathrm{C}} \mathrm{H}=\mathrm{CHCH}_{2}\right), 100.7$ (C1'), $100.6(\mathrm{C} 1), 75.5$ (C4), 74.3 (CHBz), 72.9 (C5), 72.9 (C3), 72.4 (C5'), 71.9 (C2), 71.0 (C3'), 69.2 (C2'), 67.6 ( $\left.\mathrm{CH}_{2} \mathrm{OLac}\right), 62.0$ (C6), $50.8(\mathrm{CNH}), 50.3\left(\mathrm{C} 6\right.$ '), $37.0\left(\mathrm{C}(\mathrm{O}) \underline{\mathrm{CH}}_{2}\right), 32.5\left(\mathrm{CH}=\mathrm{CHCH}_{2}\right), 32.1,31.8,29.81$ - 29.79 (m), 29.8, 29.5, 29.41, 29.37, 29.2, 29.1, 25.8, 22.8, $22.8\left(\mathrm{CH}_{2}\right), 21.0,20.9,20.75,20.74$, 20.73, 20.6 (6x C(O) $\left.\underline{\mathrm{CH}}_{3}\right), 14.24,14.18\left(2 \mathrm{x} \mathrm{CH}_{3}\right)$.

ESI-MS: $\quad$ calculated $\quad[\mathrm{M}+\mathrm{Na}]^{+}=1053.6$

found $\quad[\mathrm{M}+\mathrm{Na}]^{+}=1053.7$ 
(2S,3S,4E)-2-Eicosanamido-3-(benzoyloxy)-4-octadecen-1-yl-2,3,4-tri-O-acetyl-6-azido-6deoxy- $\beta$-D-galactopyranosyl-( $1 \rightarrow 4)-2,3,6$-tri- $O$-acetyl- $\beta$-D-glucopyranoside (46)

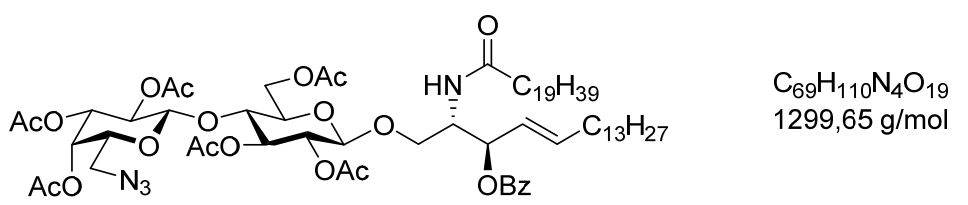

46 was synthesized as described for $\mathbf{3 4}$, starting from $58 \mathrm{mg}(0.083 \mathrm{mmol})$ ceramide $\mathbf{3 0}$ and $76 \mathrm{mg}(0.10 \mathrm{mmol})$ trichloroacetimidate 43. After purification with silica chromatography 103 mg $46(79 \mu \mathrm{mol})$ was obtained as colorless solid.

TLC: $R_{f}=0.27$ (eluent toluene/acetone 4:1)

${ }^{1} \mathbf{H}$ NMR (400 MHz, $\left.\mathrm{CDCl}_{3}\right): \delta=8.05-7.00$ (m, $2 \mathrm{H}$, aromat.), $7.60-7.53$ (m, $1 \mathrm{H}$, aromat.), $7.47-7.41$ (m, $2 \mathrm{H}$, aromat.), $5.89-5.82\left(\mathrm{~m}, 1 \mathrm{H}, \mathrm{CH}=\mathrm{CHCH}_{2}\right), 5.75$ (d, $J=9.2$ $\mathrm{Hz}, 1 \mathrm{H}, \mathrm{NH}), 5.55-5.50$ (m, $1 \mathrm{H}, \mathrm{CHOBz}), 5.48-5.42\left(\mathrm{~m}, 1 \mathrm{H}, \mathrm{C} \underline{\mathrm{H}}=\mathrm{CHCH}_{2}\right), 5.33$ (m, 1 H, H-4'), 5.15 ('t', $J=9.3 \mathrm{~Hz}, 1 \mathrm{H}, \mathrm{H}-3$ ), 5.05 (dd, $J=10.4,7.9 \mathrm{~Hz}, 1 \mathrm{H}, \mathrm{H}-2$ '), 5.00 - 4.93 (m, 1 H, H-3'), 4.86 (dd, J=9.4, 7.8 Hz, 1 H, H-2), 4.48 - 4.43 (m, 3 H, H-1', CㅌNH, H-1), 4.35 (dd, $J=11.9,1.8 \mathrm{~Hz}, 1 \mathrm{H}, \mathrm{H}-6 \mathrm{a}$ ), $4.00-3.94$ (m, $2 \mathrm{H}, \mathrm{CH}_{2} \mathrm{OLac}, \mathrm{H}-6 \mathrm{~b}$ ), 3.82 ('t', $J=9.5$ Hz, $1 \mathrm{H}, \mathrm{H}-4), 3.72$ - 3.69 (m, $1 \mathrm{H}, \mathrm{H}-5$ '), 3.62 (dd, $J=10.1,4.5$ Hz, $1 \mathrm{H}, \mathrm{CH}_{2} \mathrm{OLac}$ ), 3.56 (ddd, $J=9.8,4.9,1.9 \mathrm{~Hz}, 1 \mathrm{H}, \mathrm{H}-5), 3.45$ (dd, $J=12.7,7.3 \mathrm{~Hz}, 1 \mathrm{H}, \mathrm{H}-6 \mathrm{a}$ '), 3.23 (dd, $J=$ 12.8, $5.7 \mathrm{~Hz}, 1 \mathrm{H}, \mathrm{H}-6 \mathrm{~b}$ '), $2.16-2.14$ (m, $\left.5 \mathrm{H}, \mathrm{C}(\mathrm{O}) \mathrm{CH}_{3}, \mathrm{C}(\mathrm{O}) \mathrm{CH}_{2}\right), 2.06-2.01$ (m, $11 \mathrm{H}, 3 \mathrm{x}$ $\left.\mathrm{C}(\mathrm{O}) \mathrm{CH}_{3}, \mathrm{CH}=\mathrm{CHCH}_{2}\right), 1.96-1.95\left(\mathrm{~m}, 6 \mathrm{H}, 2 \mathrm{x} \mathrm{C}(\mathrm{O}) \mathrm{CH}_{3}\right), 1.64-1.54(\mathrm{~m}, 2 \mathrm{H}$, $\left.\mathrm{C}(\mathrm{O}) \mathrm{CH}_{2} \underline{\mathrm{C}}_{2}\right), 1.38-1.22\left(\mathrm{~m}, 54 \mathrm{H}, 27 \mathrm{x} \mathrm{CH}_{2}\right), 0.85$ (t, $\left.J=6.8 \mathrm{~Hz}, 6 \mathrm{H}, 2 \mathrm{x} \mathrm{CH}_{3}\right)$.

${ }^{13}$ C NMR (101 MHz, $\left.\mathrm{CDCl}_{3}\right): \delta=172.9,170.4,170.21,170.15,169.84,169.79,169.2,165.4$ (8x C(O)), $137.7\left(\mathrm{CH}=\underline{\mathrm{CHCH}_{2}}\right.$ ), 133.2 (C aromat.), 130.5 (C aromat.), 129.8 (2x C aromat.), 128.6 (2x C aromat.), $124.6\left(\underline{\mathrm{CH}}=\mathrm{CHCH}_{2}\right), 100.8,100.6$ (C1, C1'), 75.5 (C4), 74.3 (COBz), 72.9 (C5), 72.6 (C3), 72.4 (C5'), 71.9 (C2), 71.1 (C3'), 69.2 (C2'), 67.7 (COLac), 67.6 (C4'), 62.0 (C6), $50.8(\mathrm{CNH}), 50.4\left(\mathrm{C}^{\prime}\right), 37.0\left(\mathrm{C}(\mathrm{O}) \underline{\mathrm{CH}}_{2}\right), 32.5\left(\mathrm{CH}=\mathrm{CHCH}_{2}\right), 32.1,29.9$ - 29.8 (m), 29.73, 29.68, 29.6, 29.6 - 29.5 (m), 29.4, 29.1, 25.9, $22.8\left(\mathrm{CH}_{2}\right)$, 21.1, 20.9, 20.80, 20.79, 20.78, 20.7 (6x C(O) $\left.\mathrm{CH}_{3}\right), 14.3\left(2 \mathrm{x} \mathrm{CH}_{3}\right)$.

ESI-MS: $\quad$ calculated $\quad[\mathrm{M}+\mathrm{Na}]^{+}=1321.8$ found $\quad[\mathrm{M}+\mathrm{Na}]^{+}=1321.1$ 
(2S,3S,E)-2-Butyramido-3-hydroxy-octadec-4-en-1-yl 6-azido-6-deoxy- $\beta$-D-galactopyranosyl-(1 $\rightarrow 4)-\beta$-D-glucopyranoside $(47)$

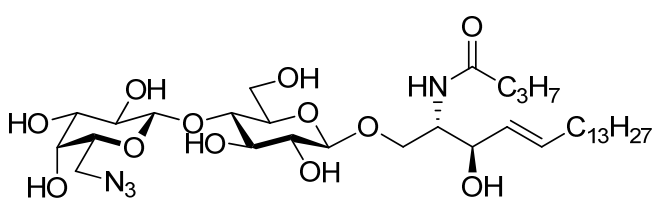

47 was prepared as described for compound 2. Starting from 44 (66.4 mg, $62 \mu \mathrm{mol}), 47$ was obtained as a colorless solid (27.6 mg, $38 \mu \mathrm{mol})$.

TLC: $R_{f}=0.34$ (eluent DCM/MeOH 4:1)

${ }^{1} \mathbf{H}$ NMR (400 MHz, CD $\left.{ }_{3} \mathrm{OD}\right): \delta=7.82(\mathrm{~d}, J=9.2 \mathrm{~Hz}, 1 \mathrm{H}, \mathrm{NH}), 5.70(\mathrm{dt}, J=13.7,6.6 \mathrm{~Hz}, 1$ $\left.\mathrm{H}, \mathrm{CH}=\mathrm{C} \mathrm{HCH}_{2}\right), 5.46\left(\mathrm{~m}, 1 \mathrm{H}, \mathrm{C} \underline{\mathrm{H}}=\mathrm{CHCH}_{2}\right), 4.39(\mathrm{~d}, J=7.3 \mathrm{~Hz}, 1 \mathrm{H}, \mathrm{H}-1$ '), $4.30(\mathrm{~d}, J=7.8$ $\mathrm{Hz}, 1 \mathrm{H}, \mathrm{H}-1$ ), 4.15 (dd, $J=10.1,4.8 \mathrm{~Hz}, 1 \mathrm{H}, \mathrm{CH}_{2} \mathrm{OLac}$ ), 4.11 - 4.07 (m, $1 \mathrm{H}$, sphingosine-

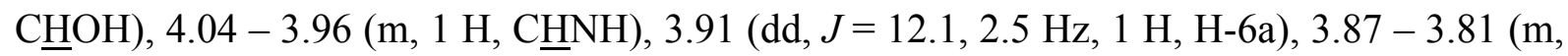
1 H, H-6b), 3.79 - 3.77 (m, 1 H, H-4'), 3.73 - 3.67 (m, 1 H, H-5'), 3.62 - 3.48 (m, 7 H, $\mathrm{CH}_{2} \mathrm{OLac}, \mathrm{H}-3, \mathrm{H}-4, \mathrm{H}-2$ ', H-3', H-6a/b'), 3.45 - 3.40 (m, 1 H, H-5), 3.30 - 3.27 (m, 1 H, H2), $2.16\left(\mathrm{t}, J=7.4 \mathrm{~Hz}, 2 \mathrm{H}, \mathrm{C}(\mathrm{O}) \mathrm{CH}_{2}\right), 2.06-1.99\left(\mathrm{~m}, 2 \mathrm{H}\left(\mathrm{CH}=\mathrm{CHC}_{2}\right), 1.67-1.56(\mathrm{~m}, 2\right.$ $\left.\mathrm{H}, \mathrm{CH}_{2}\right), 1.43-1.23\left(\mathrm{~m}, 22 \mathrm{H}, 11 \mathrm{x} \mathrm{CH}_{2}\right), 0.94\left(\mathrm{t}, J=7.4 \mathrm{~Hz}, 3 \mathrm{H}, \mathrm{CH}_{3}\right), 0.90(\mathrm{t}, J=6.9 \mathrm{~Hz}, 3$ $\left.\mathrm{H}, \mathrm{CH}_{3}\right)$.

${ }^{13} \mathrm{C}$ NMR (101 MHz, $\left.\mathrm{CD}_{3} \mathrm{OD}\right): \delta=175.9(\mathrm{C}(\mathrm{O})), 135.0\left(\mathrm{CH}=\underline{\mathrm{CHCH}_{2}}\right), 131.2\left(\underline{\mathrm{C}} \mathrm{H}=\mathrm{CHCH}_{2}\right)$, 105.0 (C1), 104.5 (C1'), 80.6, 76.4 (C5), 76.2, 74.9 (C5'), 74.8 (C2), 74.5, 73.1 (sphingosineCHOH), 72.2, 70.4 (C4'), 69.9 ( $\left.\mathrm{CH}_{2} \mathrm{OLac}\right), 61.8$ (C6), 54.8 (CHNH), 52.4 (C6'), 39.2 $\left(\mathrm{C}(\mathrm{O}) \underline{\mathrm{CH}}_{2}\right), 33.4\left(\mathrm{CH}=\mathrm{CHCH}_{2}\right), 33.0,30.78-30.73(\mathrm{~m}), 30.71,30.4,30.34,30.30,23.7,20.4$ $\left(\mathrm{CH}_{2}\right), 14.4,14.1\left(2 \mathrm{x} \mathrm{CH}_{3}\right)$.

$\begin{array}{lll}\text { ESI-MS: } & \text { calculated } & {[\mathrm{M}+\mathrm{Na}]^{+}=741.4,[\mathrm{M}-\mathrm{H}]^{-}=717.4} \\ & \text { found } & {[\mathrm{M}+\mathrm{Na}]^{+}=742.0,[\mathrm{M}-\mathrm{H}]^{-}=718.0}\end{array}$

HR-ESI-MS: calculated $\quad[\mathrm{M}+\mathrm{H}]^{+}=719.44370$ found $[\mathrm{M}+\mathrm{H}]^{+}=719.44255$ 


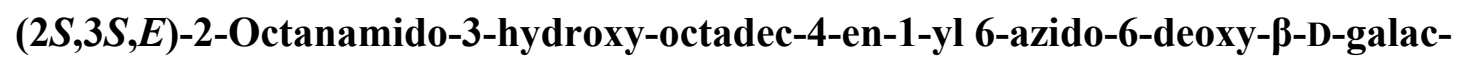
topyranosyl-(1 $\rightarrow 4)-\beta$-D-glucopyranoside $(48)$

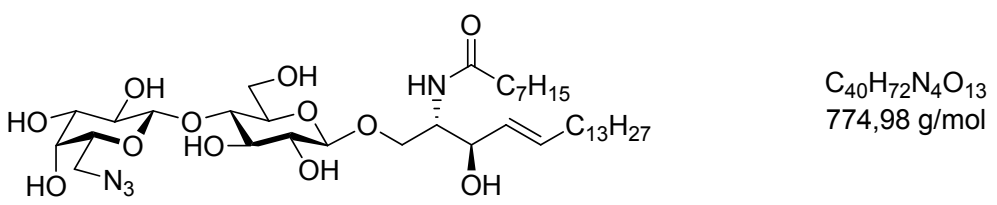

Deacylation of $\mathbf{4 5}(91 \mathrm{mg}, 80 \mu \mathrm{mol})$ to yield $\mathbf{4 8}$ was carried out as described for compound 2. After purification by silica column chromatography (eluent DCM/MeOH 9:1 to 87:13) 48 was obtained as a colorless solid (46 mg, $59 \mu \mathrm{mol}, 74 \%$ ).

TLC: $R_{f}=0.44$ (eluent DCM/MeOH 4:1)

${ }^{1}$ H NMR (400 MHz, CD 3 OD): $\delta=7.84(\mathrm{~d}, J=9.1 \mathrm{~Hz}, 1 \mathrm{H}, \mathrm{NH}), 5.69(\mathrm{dt}, J=14.8,6.7 \mathrm{~Hz}, 1$ $\left.\mathrm{H}, \mathrm{CH}=\mathrm{CHCH}_{2}\right), 5.45\left(\mathrm{dd}, J=15.3,7.6 \mathrm{~Hz}, 1 \mathrm{H}, \mathrm{CH}=\mathrm{CHCH}_{2}\right), 4.38(\mathrm{~d}, J=7.3 \mathrm{~Hz}, 1 \mathrm{H}, \mathrm{H}-$ 1'), 4.30 (d, $J=7.8 \mathrm{~Hz}, 1 \mathrm{H}, \mathrm{H}-1$ ), 4.17 (dd, $J=10.0,4.6 \mathrm{~Hz}, 1 \mathrm{H}, \mathrm{CH}_{2} \mathrm{OLac}$ ), 4.08 ('t', $J=$

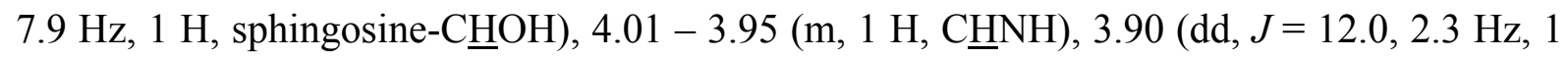
H, H-6a), 3.84 (dd, $J=12.1,4.2$ Hz, 1 H, H-6b), 3.78 (dd, $J=2.8,0.5$ Hz, 1 H, H-4'), 3.72 3.69 (m, 1 H, H-5'), 3.62 - 3.52 (m, 6 H, H-3, H-4, H-2', H-6a/b', $\mathrm{CH}_{2} \mathrm{OLac}$ ), 3.50 (dd, J= 9.7, 3.0 Hz, 1 H, H-3'), 3.45 - 3.40 (m, 1 H, H-5), 3.32 - 3.29 (obscured, $1 \mathrm{H}, \mathrm{H}-2$ ), 2.17 (t, J $\left.=7.6 \mathrm{~Hz}, 2 \mathrm{H}, \mathrm{C}(\mathrm{O}) \mathrm{CH}_{2}\right), 2.06-2.00\left(\mathrm{~m}, 2 \mathrm{H}, \mathrm{CH}=\mathrm{CHC}_{2}\right), 1.62-1.55(\mathrm{~m}, 2 \mathrm{H}$, $\mathrm{C}(\mathrm{O}) \mathrm{CH}_{2} \mathrm{C}_{2}$ ) $, 1.42-1.25\left(\mathrm{~m}, 30 \mathrm{H}, 15 \mathrm{x} \mathrm{CH}_{2}\right), 0.91$ (t, $\left.J=6.8 \mathrm{~Hz}, 3 \mathrm{H}, \mathrm{CH}_{3}\right), 0.90$ (t, $J=6.8$ $\left.\mathrm{Hz}, \mathrm{CH}_{3}\right)$.

${ }^{13}$ C NMR (101 MHz, $\left.\mathrm{CD}_{3} \mathrm{OD}\right): \delta=176.0 \mathrm{C}(\mathrm{O}), 135.0\left(\mathrm{CH}=\underline{\mathrm{C}} \mathrm{HCH}_{2}\right), 131.3\left(\underline{\mathrm{C}} \mathrm{H}=\mathrm{CHCH}_{2}\right)$, 105.0 (C1'), 104.5 (C1), 80.6 (C4), 76.4 (C5), 76.2, 75.9 (C5'), 74.9 (C2), 74.5 (C3'), 73.0 (sphingosine-CHOH), 72.24, 70.4 (C4'), 49.9 ( $\left.\mathrm{CH}_{2} \mathrm{OLac}\right), 61.8$ (C6), 54.7 (CNH), 52.4 (C6'), $37.4\left(\mathrm{C}(\mathrm{O}) \underline{\mathrm{CH}}_{2}\right), 33.4\left(\mathrm{CH}=\mathrm{CHCH}_{2}\right), 33.4,33.1(2 \mathrm{x} \mathrm{CH}), 33.0,30.82-30.77\left(\mathrm{~m}, \mathrm{CH}_{2}\right)$, $30.72,30.5,30.41,30.40,30.28,27.2,23.75,23.73\left(8 \mathrm{x} \mathrm{CH}_{2}\right), 14.47,14.43\left(2 \mathrm{x} \mathrm{CH}_{3}\right)$.

HR-ESI-MS: calculated $[\mathrm{M}+\mathrm{H}]^{+}=775.50630$ found $\quad[\mathrm{M}+\mathrm{H}]^{+}=775.50412$ 


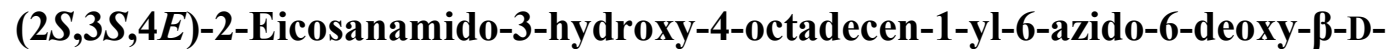

\section{galactopyranosyl-( $1 \rightarrow 4)-\beta$-D-glucopyranoside $(49)$}

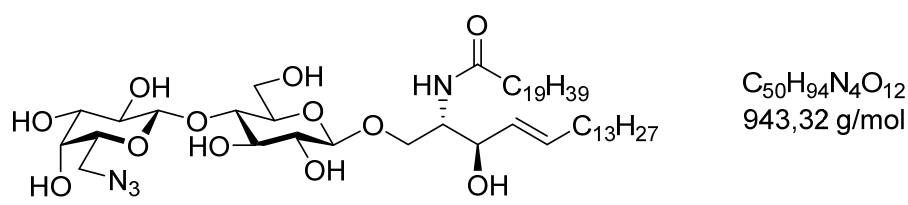

Glycolipid 49 was synthesized as described for 2, starting from $102 \mathrm{mg}$ 46. After purification with silica chromatography (eluent DCM/MeOH 8:1, then 7:1) $40 \mathrm{mg} 49$ (43 $\mu \mathrm{mol}, 52 \%$ over two steps) was obtained as colorless solid.

TLC: $R_{f}=0.26$ (eluent DCM/MeOH 6:1)

${ }^{1}$ H NMR (400 MHz, DMSO): $\delta=7.47(\mathrm{~d}, J=9.0 \mathrm{~Hz}, 1 \mathrm{H}, \mathrm{NH}), 5.53(\mathrm{dt}, J=15.0,6.6 \mathrm{~Hz}, 1$ $\left.\mathrm{H}, \mathrm{CH}=\mathrm{CHCH}_{2}\right), 5.35\left(\mathrm{dd}, J=15.3,7.1 \mathrm{~Hz}, 1 \mathrm{H}, \underline{\mathrm{C}} \underline{\mathrm{H}}=\mathrm{CHCH}_{2}\right), 5.18-5.13(\mathrm{~m}, 2 \mathrm{H}, 2-\mathrm{OH}$, 2'-OH), $4.89-4.85$ (m, 2 H, 3'-OH, sphingosine-OH), 4.76 (d, J=4.7 Hz, 1 H, 3-OH), 4.56 (t, $J=5.9 \mathrm{~Hz}, 1 \mathrm{H}, 6-\mathrm{OH}), 4.40$ (s, $\left.1 \mathrm{H}, 4^{\prime}-\mathrm{OH}\right), 4.30-4.27$ (m, $\left.1 \mathrm{H}, \mathrm{H}-1\right), 4.16$ (d, J = 7.8 Hz, $1 \mathrm{H}, \mathrm{H}-1$ ') 3.97 (dd, $J=10.0,4.6 \mathrm{~Hz}, 1 \mathrm{H}, \mathrm{CH}_{2} \mathrm{OLac}$ ), 3.91 - 3.84 (m, $1 \mathrm{H}$, sphingosine-

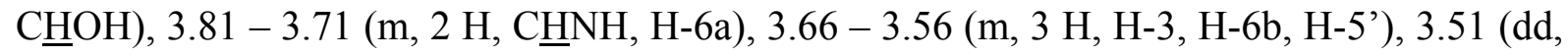
$J=12.8,4.2$ Hz, 1 H, H-6a'), 3.46 - 3.40 (m, 2 H, H-6b', $\mathrm{CH}_{2} \mathrm{OLac}$ ), 3.38 - 3.28 (m, 5 H, H2, H-4, H-5, H-3', H-4'), 3.08 - 3.01 (m, 1 H, H-2'), 2.02 (t, J=7.4 Hz, 2 H, C(O)CH $)_{2}, 1.97$ $-1.89\left(\mathrm{~m}, 2 \mathrm{H}, \mathrm{CH}=\mathrm{CHCH}_{2}\right), 1.48-1.38\left(\mathrm{~m}, 2 \mathrm{H}, \mathrm{C}(\mathrm{O}) \mathrm{CH}_{2} \mathrm{CH}_{2}\right), 1.31-1.17(\mathrm{~m}, 54 \mathrm{H}, 27 \mathrm{x}$ $\left.\mathrm{CH}_{2}\right), 0.85\left(\mathrm{t}, J=6.8 \mathrm{~Hz}, 6 \mathrm{H}, 2 \mathrm{x} \mathrm{CH}_{3}\right)$.

${ }^{13}$ C NMR (101 MHz, DMSO): $\delta=171.8(\mathrm{C}(\mathrm{O})), 131.4\left(2 \mathrm{x} \mathrm{C}, \underline{\mathrm{CH}}=\underline{\mathrm{C}} \mathrm{HCH}_{2}\right), 103.6(\mathrm{C} 1$ '), 103.3 (C1), 79.4 (C3'), 74.8 (C5), 74.34 (C4'), 73.28 (C2'), 73.1 (C5'), 70.73 (C2), 70.70 (sphingosine-COH), $70.2(\mathrm{C} 4), 69.2\left(\mathrm{CH}_{2} \mathrm{OLac}\right), 68.7(\mathrm{C} 3), 60.2(\mathrm{C} 6), 53.0(\mathrm{CNH}), 51.1$ (C6'), $35.6\left(\mathrm{C}(\mathrm{O}) \underline{\mathrm{CH}}_{2}\right), 31.8\left(\mathrm{CH}=\mathrm{CH} \underline{\mathrm{H}}_{2}\right), 31.31,31.28,29.2$ - 29.0 (m), 28.8 - 28.7 (m), 25.4, $22.09-22.07\left(\mathrm{~m}, \mathrm{CH}_{2}\right), 13.9\left(2 \mathrm{x} \mathrm{CH}_{3}\right)$.

MALDI-MS: calculated $[\mathrm{M}+\mathrm{Na}]^{+}=965.7,[\mathrm{M}+\mathrm{K}]^{+}=981.7$ found $\quad[\mathrm{M}+\mathrm{Na}]^{+}=965.8,[\mathrm{M}+\mathrm{K}]^{+}=981.8$

HR-ESI-MS: calculated $\quad[\mathrm{M}+\mathrm{H}]^{+}=819.53251$ found $\quad[\mathrm{M}+\mathrm{H}]^{+}=819.53168$ 


\section{Additional Structures}

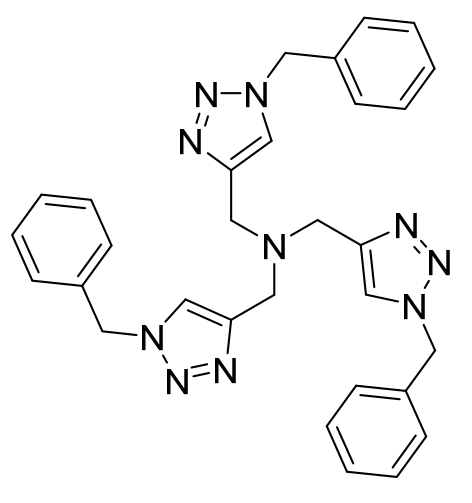

TBTA

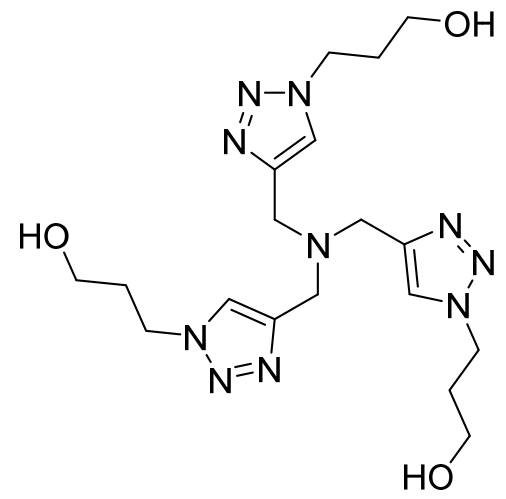

THPTA

Figure S1. Structures of TBTA and THPTA. 


\section{Incorporation of Azidolactosylceramides 47 and 48 at Different}

\section{Temperatures}

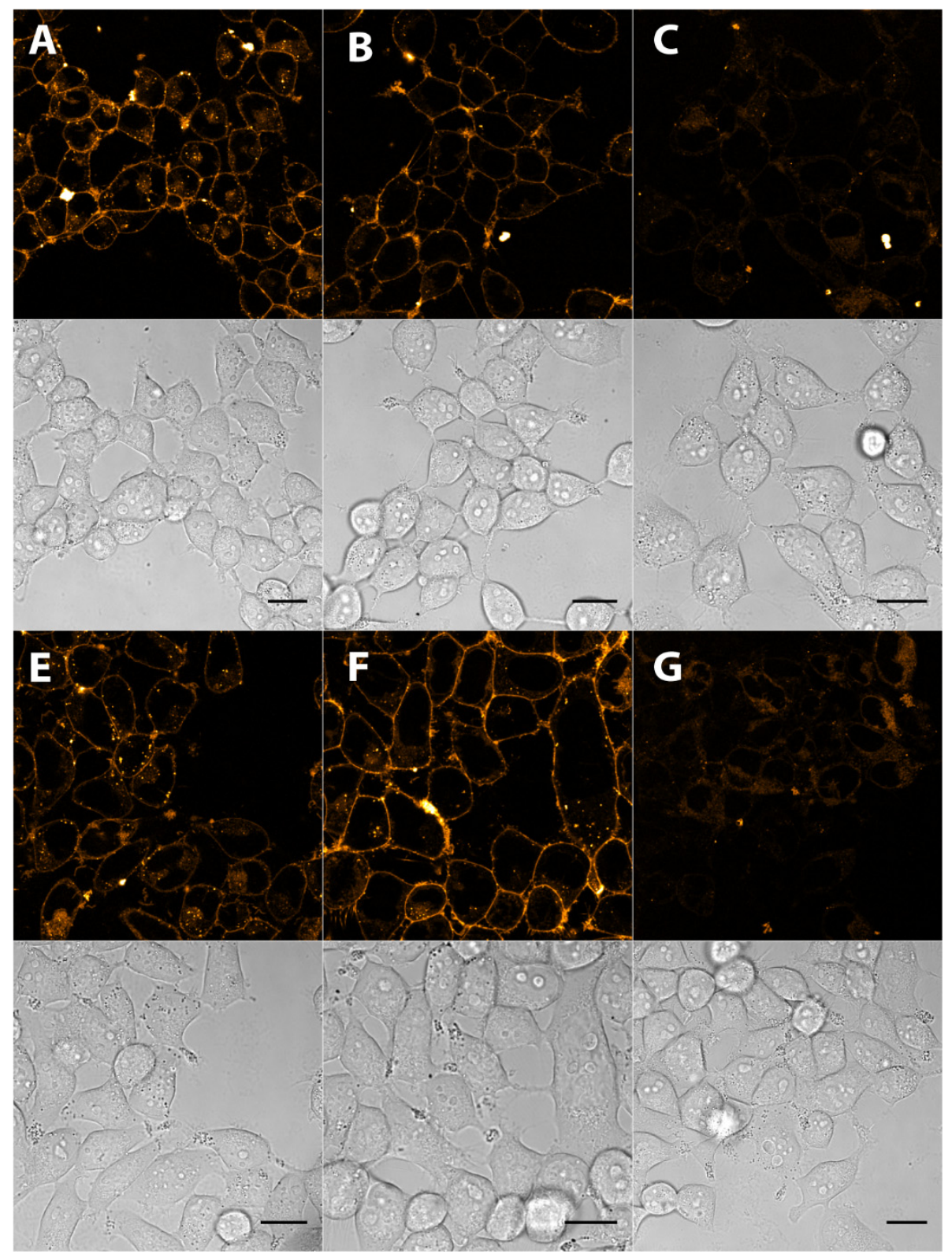

Figure S2. Cell experiments with azide-labeled lactosylceramides 47 and $\mathbf{4 8}$ at different temperatures. HEK $293 \mathrm{~T}$ cells were treated with $10 \mu \mathrm{M} 47$ (A, E), $10 \mu \mathrm{M} 48(\mathrm{~B}, \mathrm{~F})$ or without glycolipid $(\mathrm{C}, \mathrm{G})$ for $30 \mathrm{~min}$ at $4{ }^{\circ} \mathrm{C}$ (A-C) or at ambient temperature (E-G) followed by labeling with $2 \mu \mathrm{M}$ DIBO-lissamine 6. Scale bar: $20 \mu \mathrm{m}$. 


\section{Analysis by Flow Cytometry}

$10^{6}$ HEK 293T cells were seeded in 6-well plates that had been coated with $1 \mu \mathrm{g} \mathrm{mL}^{-1}$ fibronectin and $10 \mu \mathrm{g} \mathrm{mL}^{-1}$ poly-L-lysine in PBS for $1 \mathrm{~h}$ at $37^{\circ} \mathrm{C}$ and grown for $16 \mathrm{~h}$ in DMEM $+10 \% \mathrm{CS}$ at $37{ }^{\circ} \mathrm{C}$. Incubation with glycosphingolipids and labeling reactions were carried out as described in the experimental part of this publication. After fluorescence labeling, cells were washed twice with PBS and harvested by treatment with $1 \mathrm{~mL}$ trypsin/EDTA solution for $1 \mathrm{~min}$ at $37^{\circ} \mathrm{C}$. After addition of $1 \mathrm{~mL}$ DMEM $+10 \% \mathrm{CS}$, cells were centrifuged for $3 \mathrm{~min}$ at $800 \mathrm{rpm}$ and suspended in $1200 \mu \mathrm{L}$ FACS buffer (PBS $+5 \%$ heat inactivated fetal calf serum $+0.1 \% \mathrm{NaN}_{3}$ ). Approximately $10^{5}$ cells of each sample were analyzed by flow cytometry (BD Biosciences LSRII with FACSDiva software, analysis with FlowJo software).

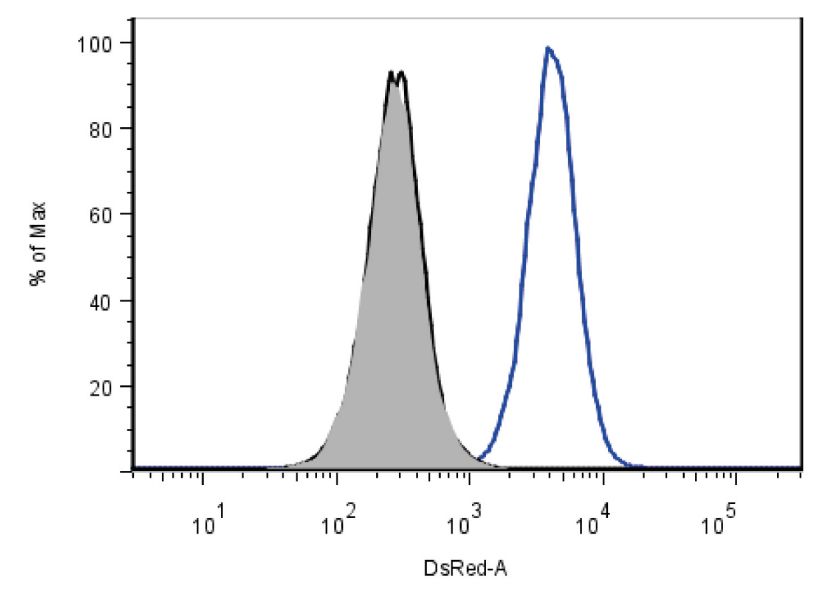

Figure S3. Flow cytometry with Streptavidin-AlexaFluor-647-labeled cells. Blue curve: HEK 293T cells were incubated with $10 \mu \mathrm{M} 48\left(30 \mathrm{~min}, 0^{\circ} \mathrm{C}\right), 30 \mu \mathrm{M}$ DIBO-biotin $(\mathbf{5 0})\left(30 \mathrm{~min}, 0^{\circ} \mathrm{C}\right)$ and streptavidin-AlexaFluor647 (30 min, $0^{\circ} \mathrm{C}$ ). Grey: untreated HEK 293T cells. Black: cells were treated with DIBO-biotin (50) and Streptavidin-AlexaFluor-647. The x-axis shows the fluorescence intensity of AlexaFluor-647, whereas the y-axis represents the relative number of cells. 


\section{References}

(1) Yang, P.-Y., Liu, K., Ngai, M. H., Lear, M. J., Wenk, M. R., and Yao, S. Q. (2010) Activity-Based Proteome Profiling of Potential Cellular Targets of Orlistat - An FDAApproved Drug with Anti-Tumor Activities. J. Am. Chem. Soc. 132, 656-666.

(2) Nguyen, T., and Francis, M. B. (2003) Practical Synthetic Route to Functionalized Rhodamine Dyes. Org. Lett. 5, 3245-3248.

(3) Loka, R. S., Sadek, C. M., Romaniuk, N. A., and Cairo, C. W. (2010) Conjugation of Synthetic N-Acetyl-Lactosamine to Azide-Containing Proteins Using the Staudinger Ligation. Bioconjugate Chem. 21, 1842-1849.

(4) Ning, X., Guo, J., Wolfert, Margreet A., and Boons, G.-J. (2008) Visualizing Metabolically Labeled Glycoconjugates of Living Cells by Copper-Free and Fast Huisgen Cycloadditions. Angew. Chem., Int. Ed. 47, 2253-2255.

(5) Anderson, S. (2008) Surfaces for Immobilization of N-Terminal Cysteine Derivatives via Native Chemical Ligation. Langmuir 24, 13962-13968. 


\section{NMR Spectra}
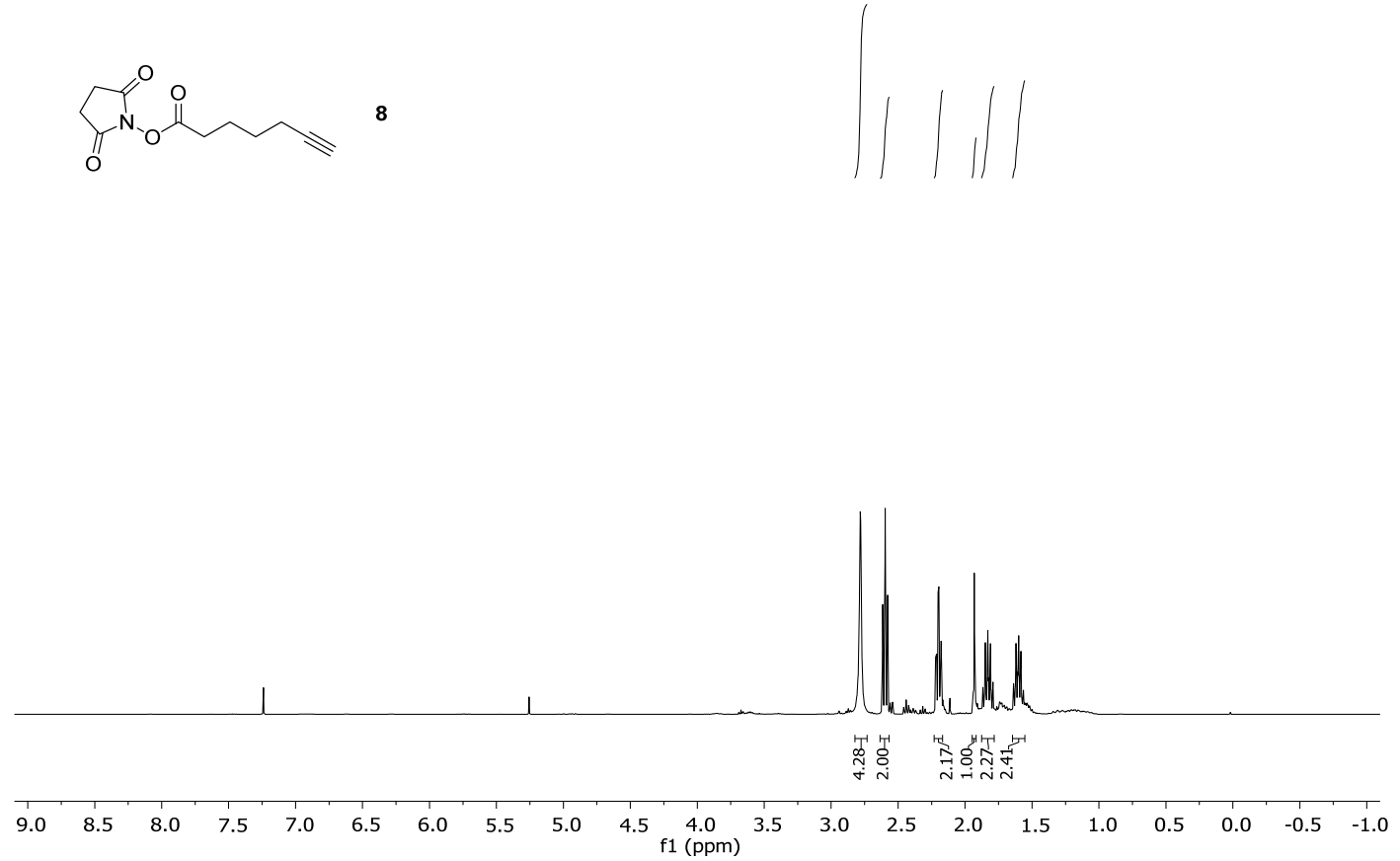

${ }^{1} \mathrm{H}$ NMR spectrum $\left(400 \mathrm{MHz}, \mathrm{CDCl}_{3}\right.$ ) of compound 8 .
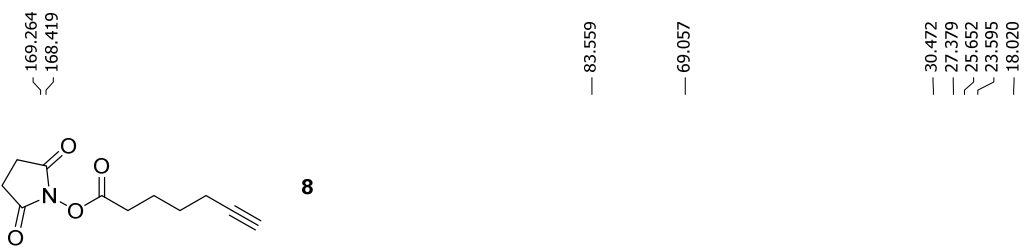

8

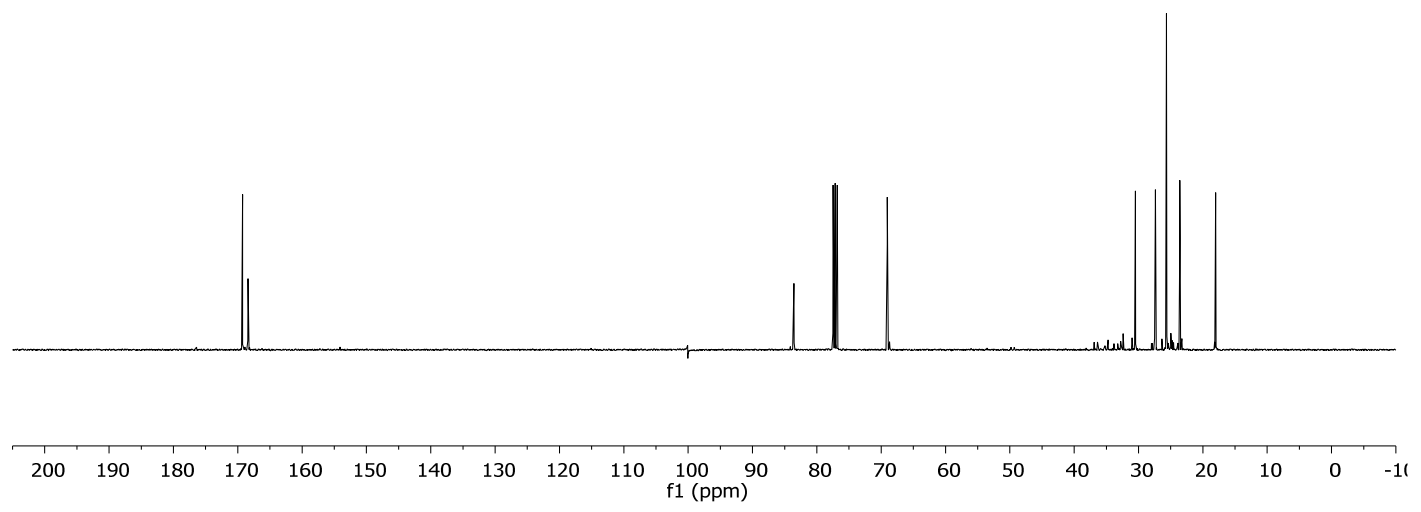

${ }^{13} \mathrm{C}$ NMR spectrum $\left(100.6 \mathrm{MHz}, \mathrm{CDCl}_{3}\right)$ of compound 8 . 

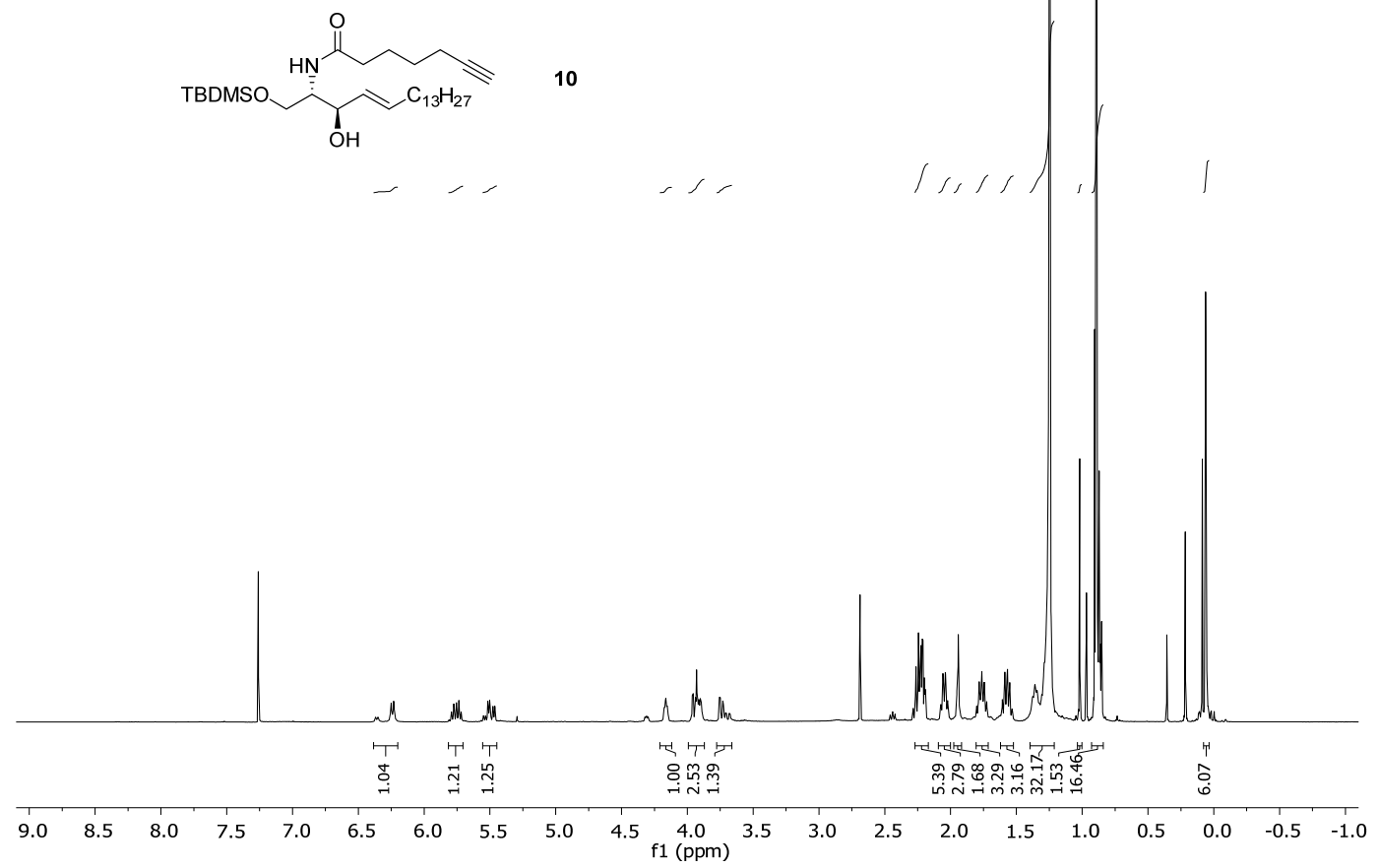

${ }^{1} \mathrm{H}$ NMR spectrum $\left(250 \mathrm{MHz}, \mathrm{CDCl}_{3}\right)$ of compound $\mathbf{1 0}$.
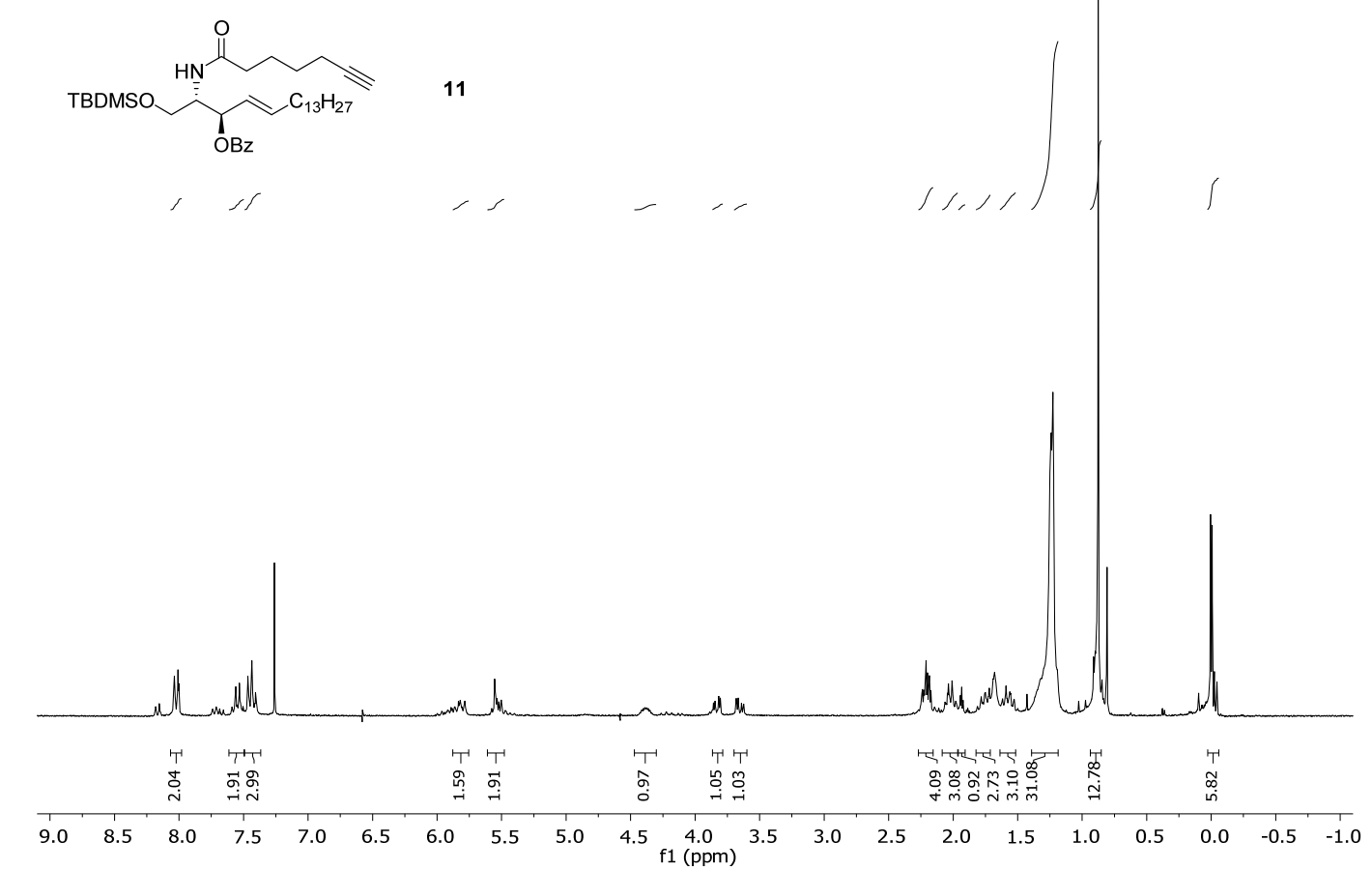

${ }^{1} \mathrm{H}$ NMR spectrum $\left(250 \mathrm{MHz}, \mathrm{CDCl}_{3}\right)$ of compound $\mathbf{1 1}$. 


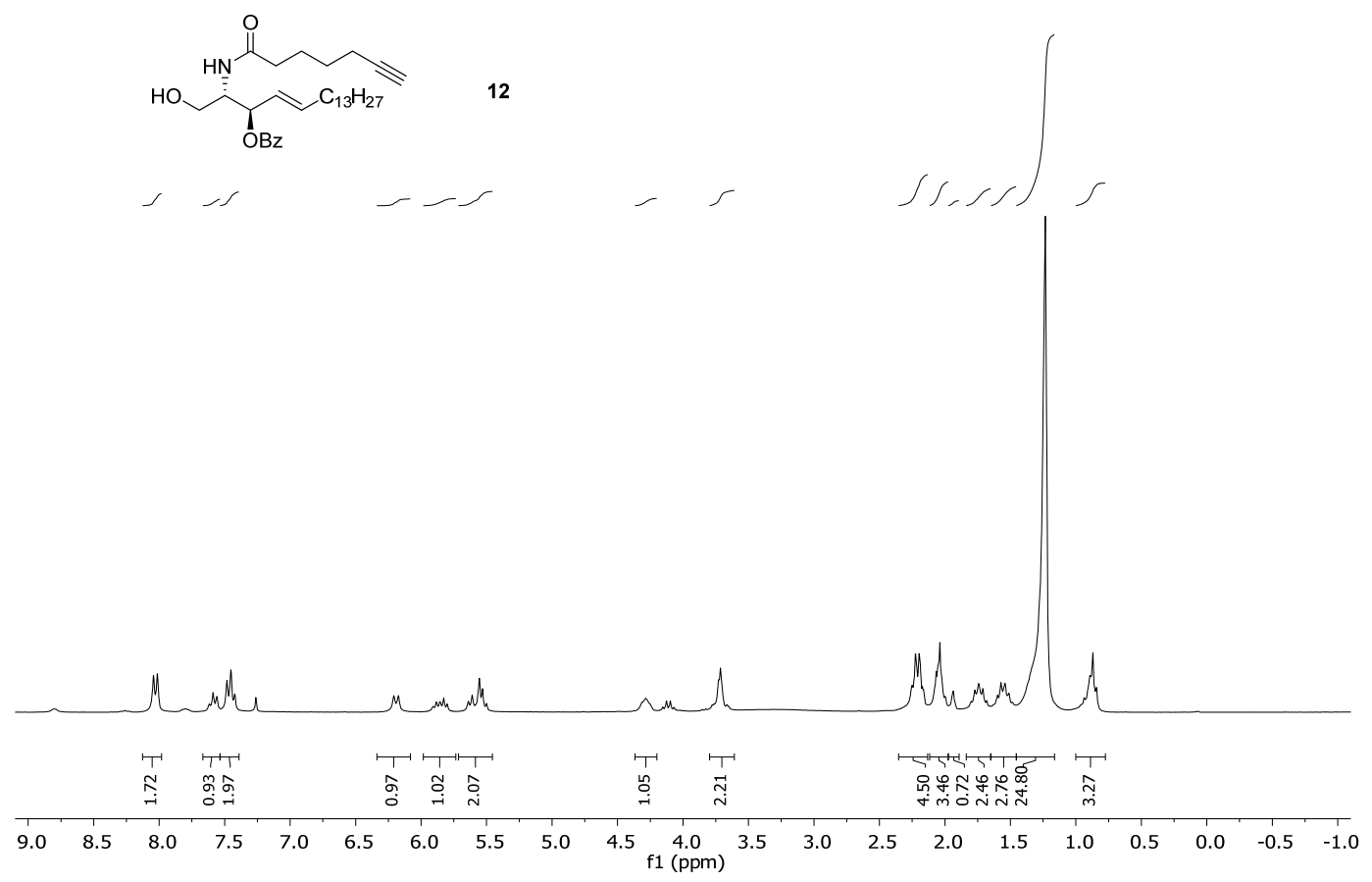

${ }^{1} \mathrm{H}$ NMR spectrum (250 MHz, $\mathrm{CDCl}_{3}$ ) of compound 12.

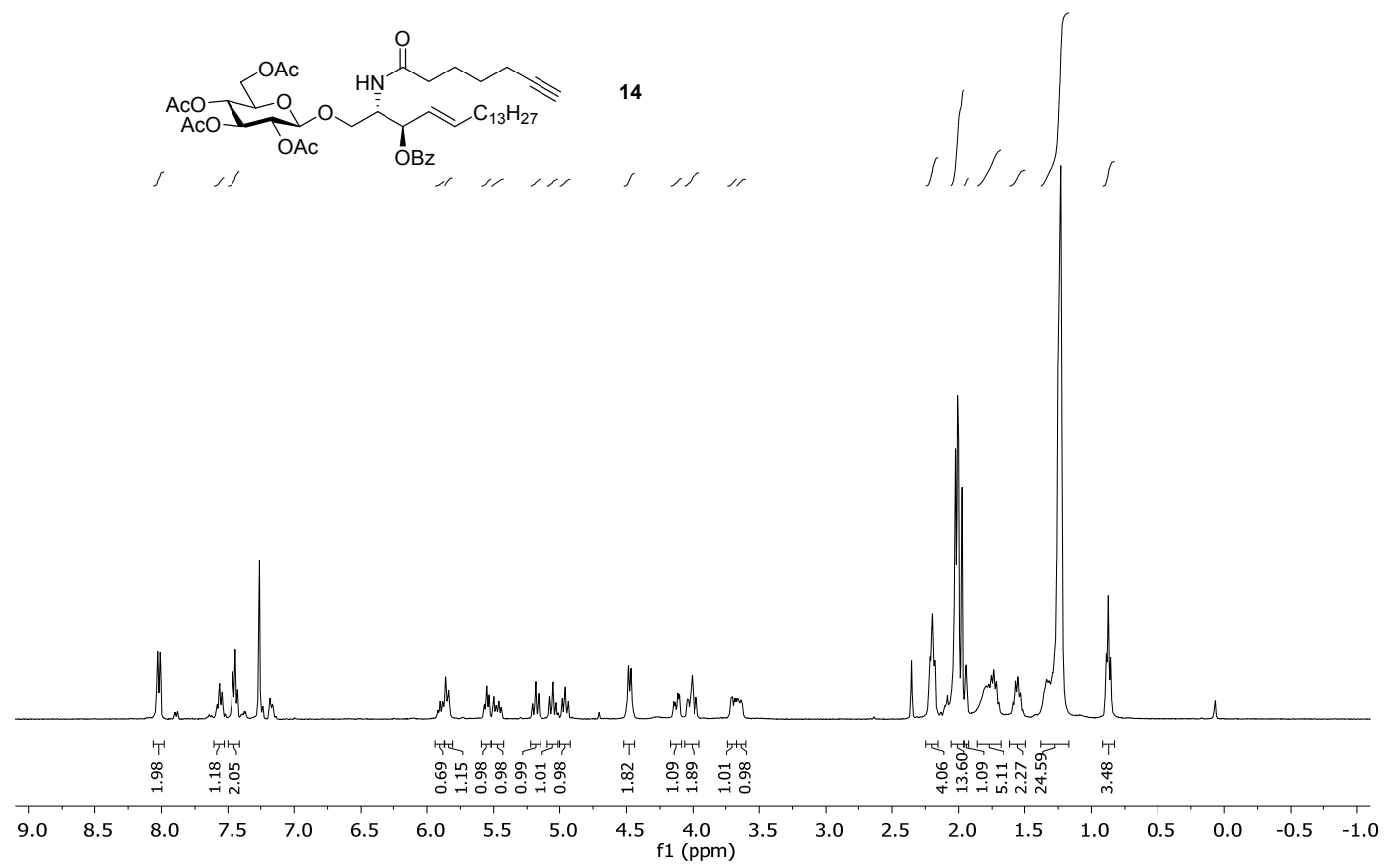

${ }^{1} \mathrm{H}$ NMR spectrum $\left(250 \mathrm{MHz}, \mathrm{CDCl}_{3}\right)$ of compound 14. 


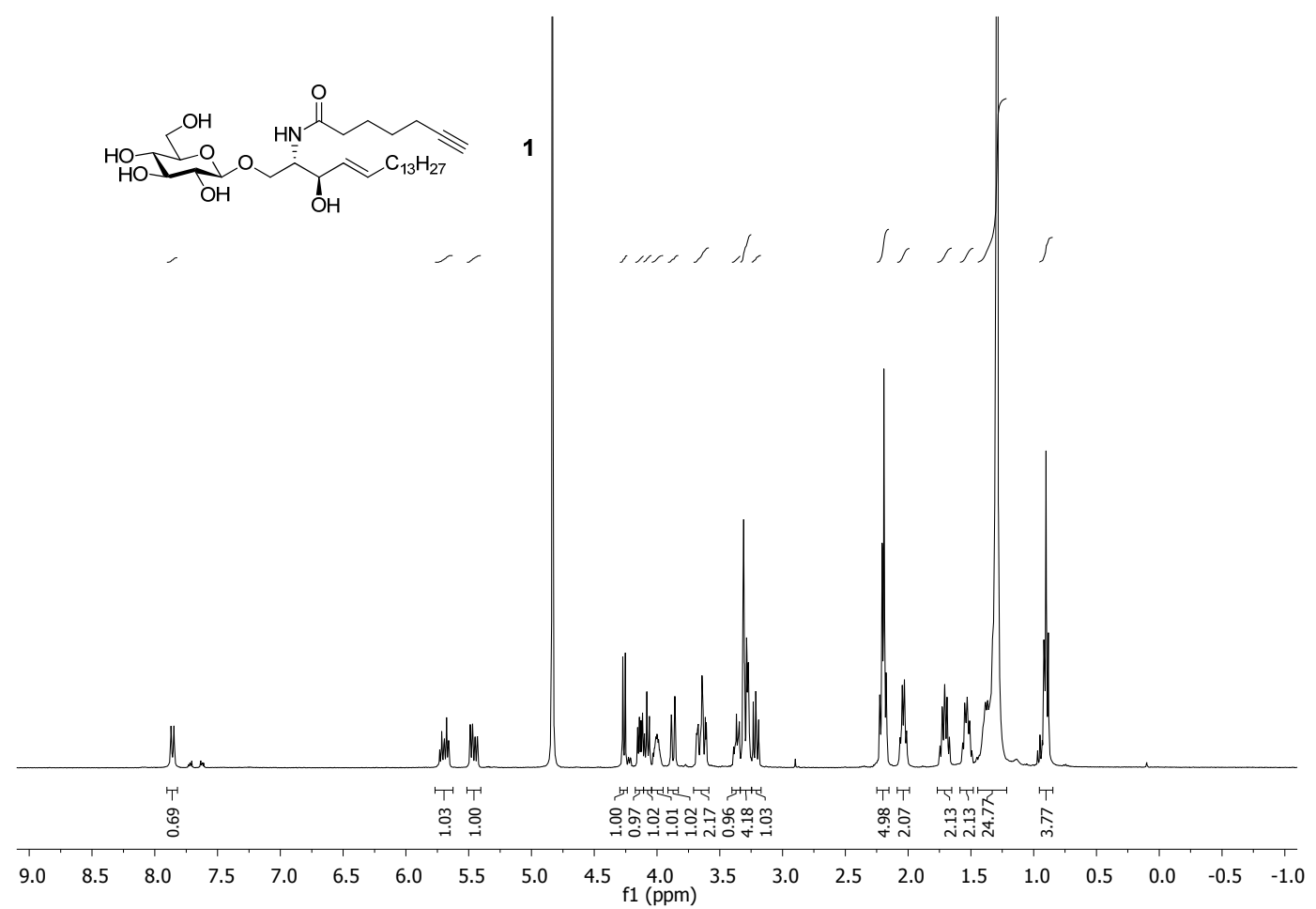

${ }^{1} \mathrm{H}$ NMR spectrum (400 MHz, $\left.\mathrm{CD}_{3} \mathrm{OD}\right)$ of compound $\mathbf{1}$.
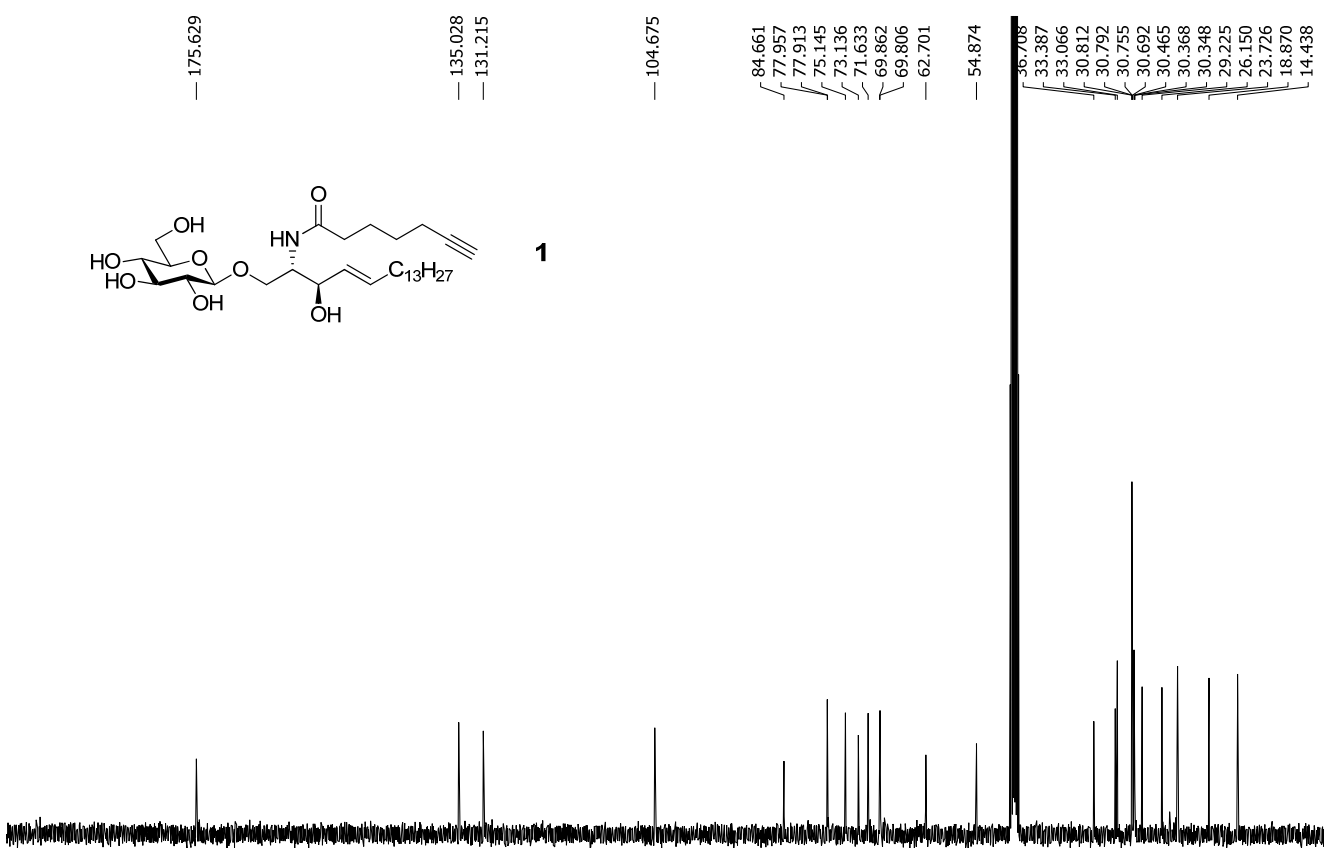

$\begin{array}{lllllllllllllllllllll}200 & 190 & 180 & 170 & 160 & 150 & 140 & 130 & 120 & 110 & 100 & 90 & 80 & 70 & 60 & 50 & 40 & 30 & 20 & 10 & 0\end{array}$

${ }^{13} \mathrm{C}$ NMR spectrum $\left(100.6 \mathrm{MHz}, \mathrm{CD}_{3} \mathrm{OD}\right)$ of compound $\mathbf{1}$. 


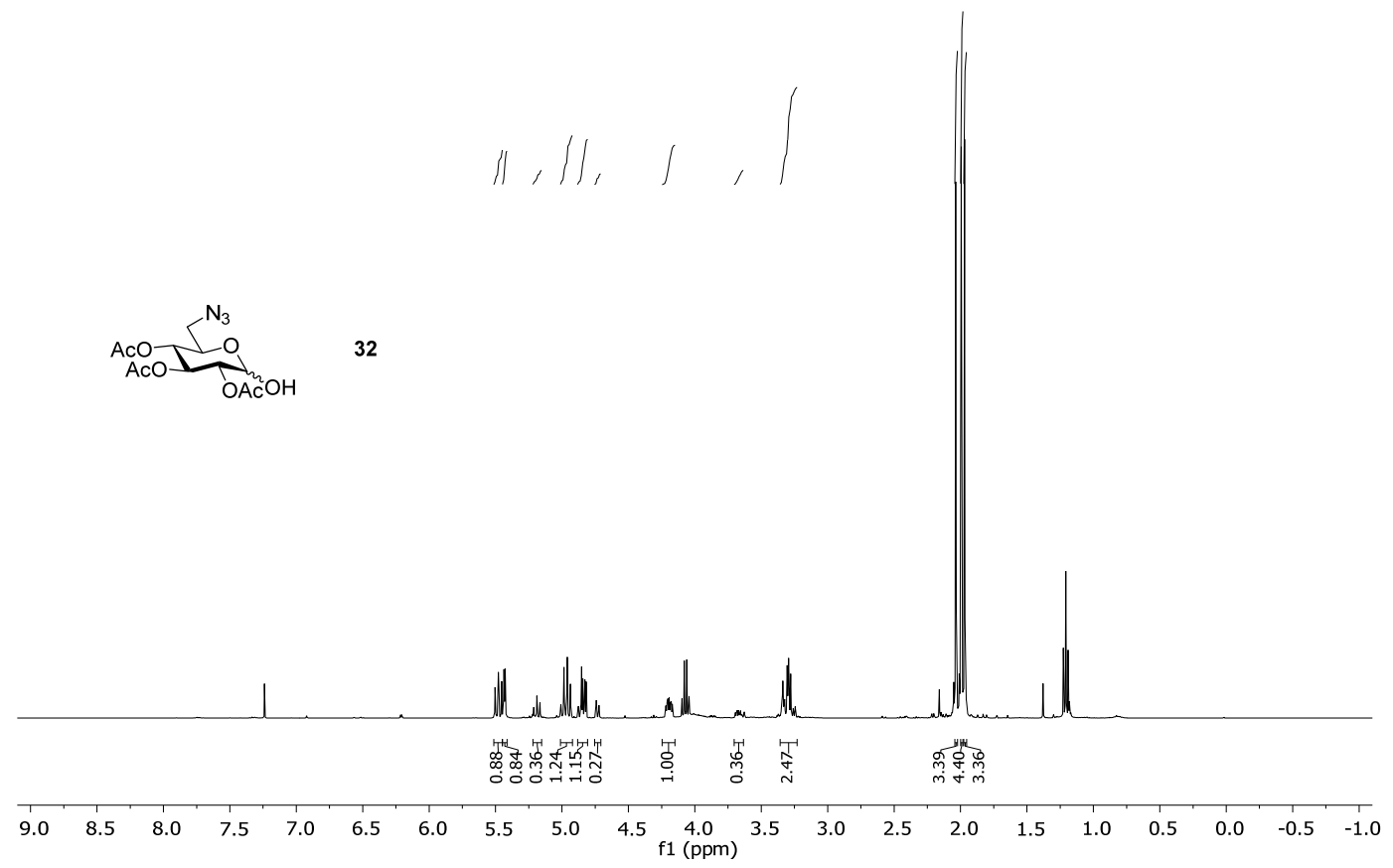

${ }^{1} \mathrm{H}$ NMR spectrum $\left(400 \mathrm{MHz}, \mathrm{CDCl}_{3}\right)$ of compound $\mathbf{3 2}$.

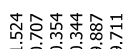

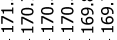

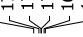

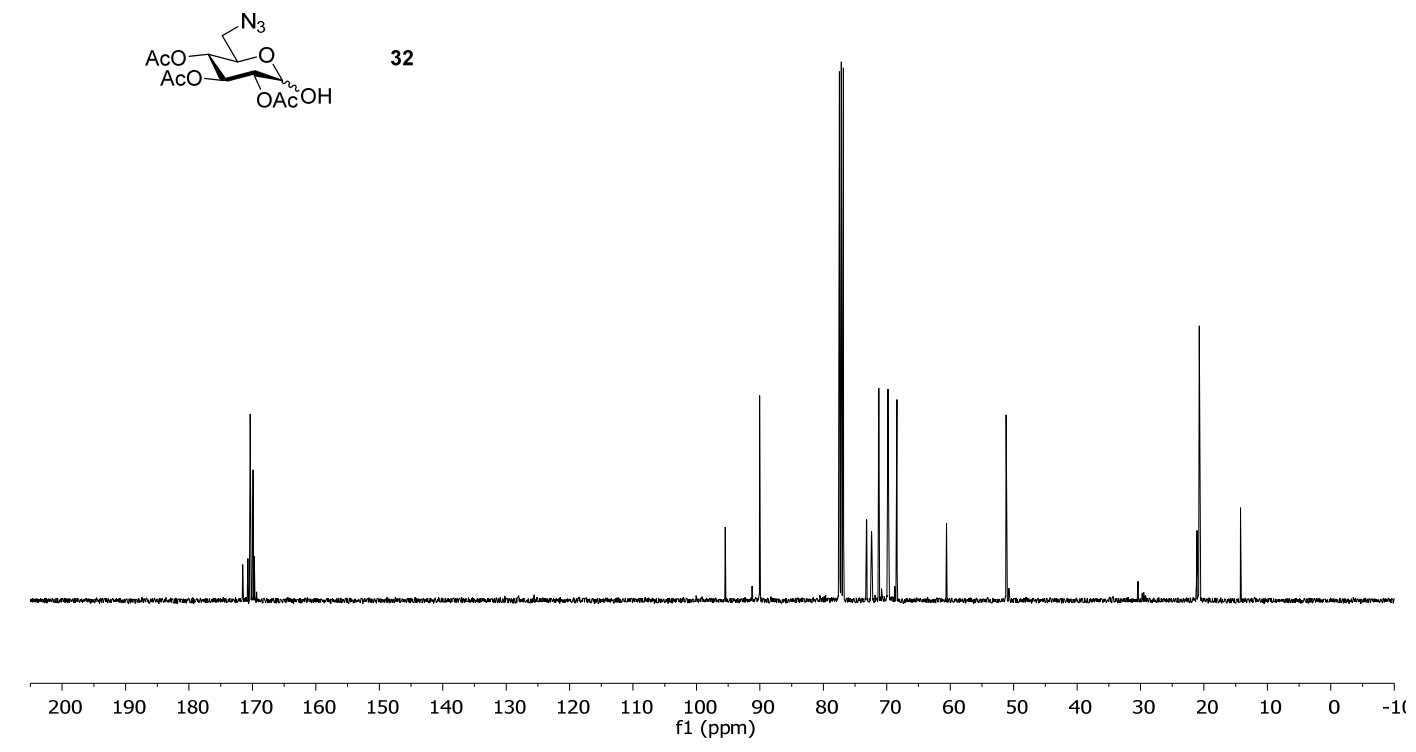

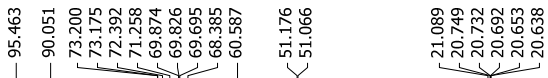

32

${ }^{13} \mathrm{C}$ NMR spectrum $\left(100.6 \mathrm{MHz}, \mathrm{CDCl}_{3}\right)$ of compound 32. 


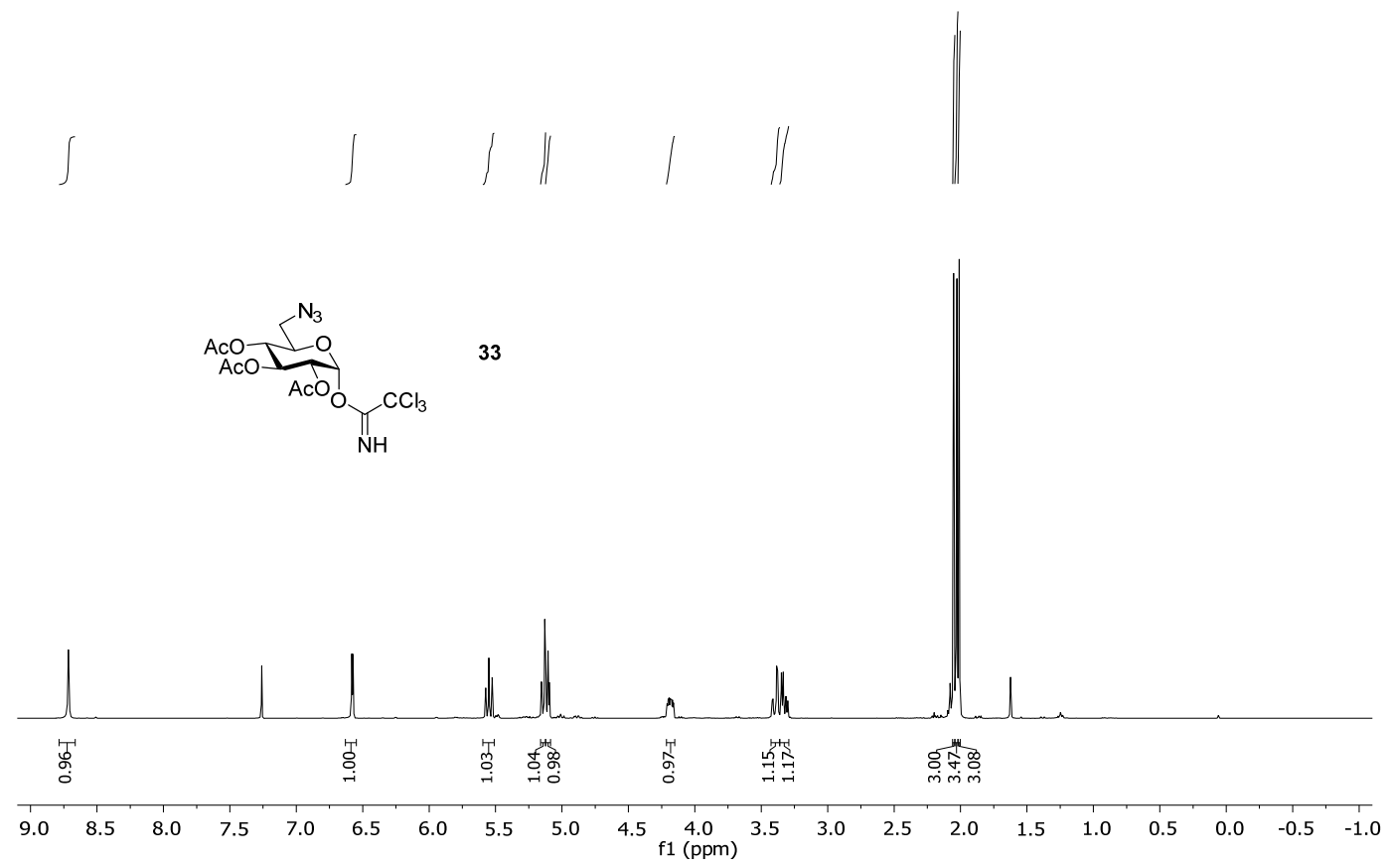

${ }^{1} \mathrm{H}$ NMR spectrum (400 MHz, $\mathrm{CDCl}_{3}$ ) of compound 33.
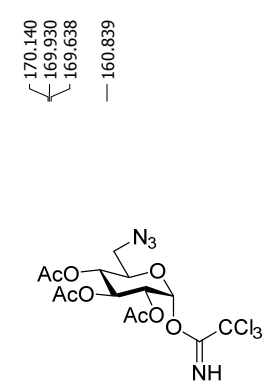

33

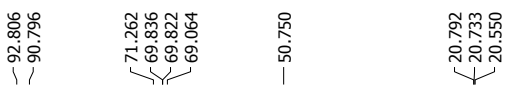

11 तो ।

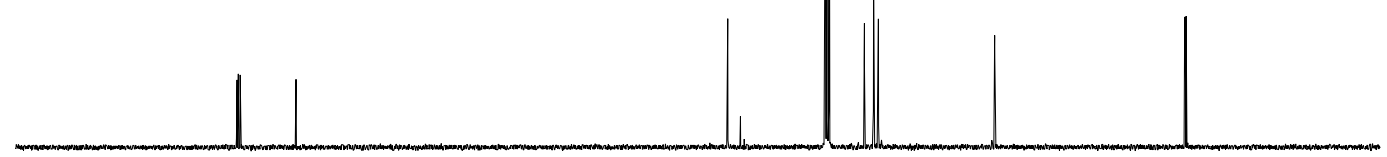

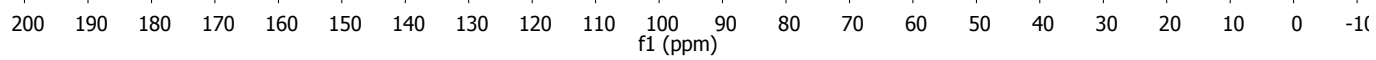

${ }^{13} \mathrm{C}$ NMR spectrum $\left(100.6 \mathrm{MHz}, \mathrm{CDCl}_{3}\right)$ of compound 33. 


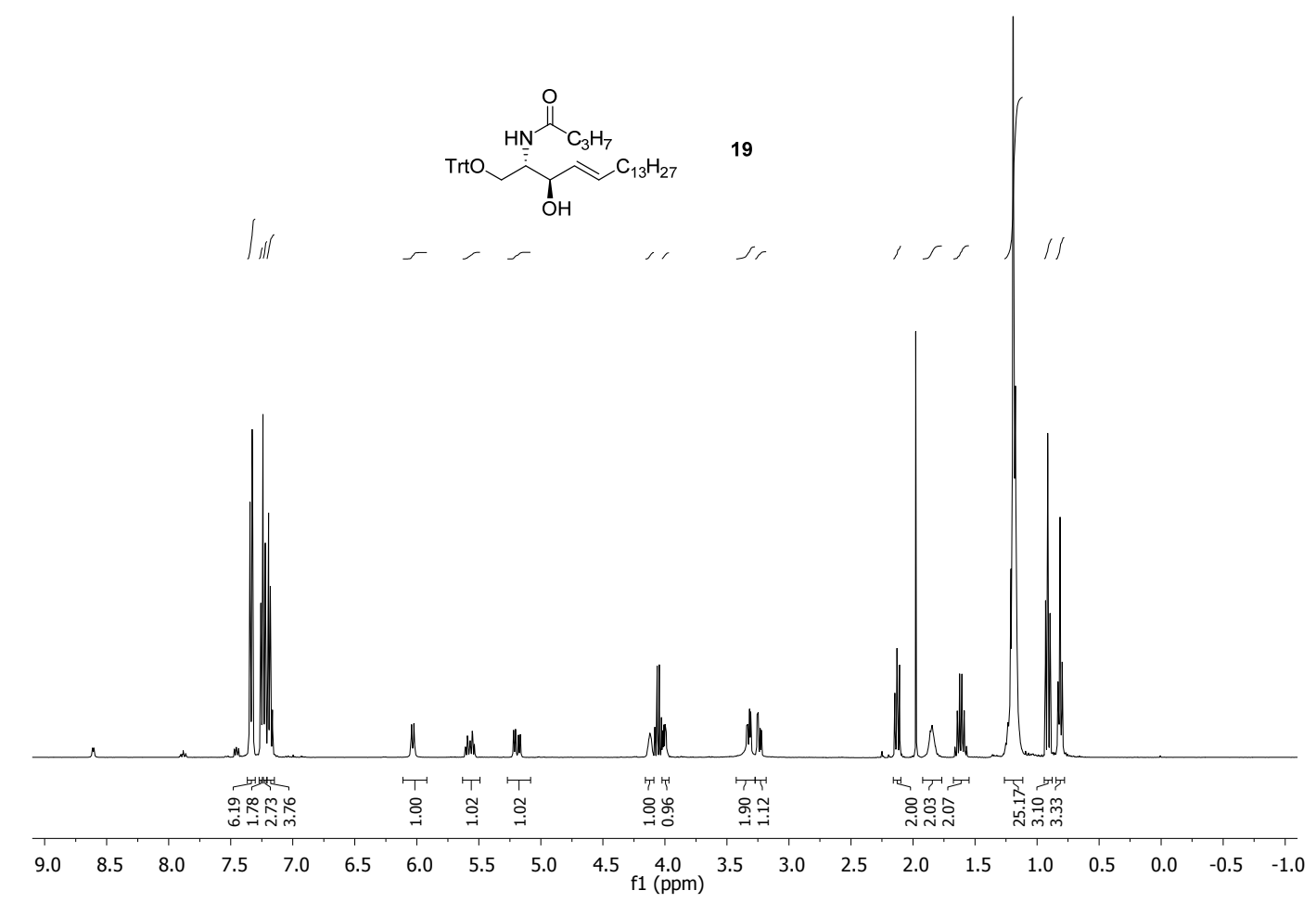

${ }^{1} \mathrm{H}$ NMR spectrum (400 MHz, $\mathrm{CDCl}_{3}$ ) of compound 19 (containing traces of pyridine and ethyl acetate).

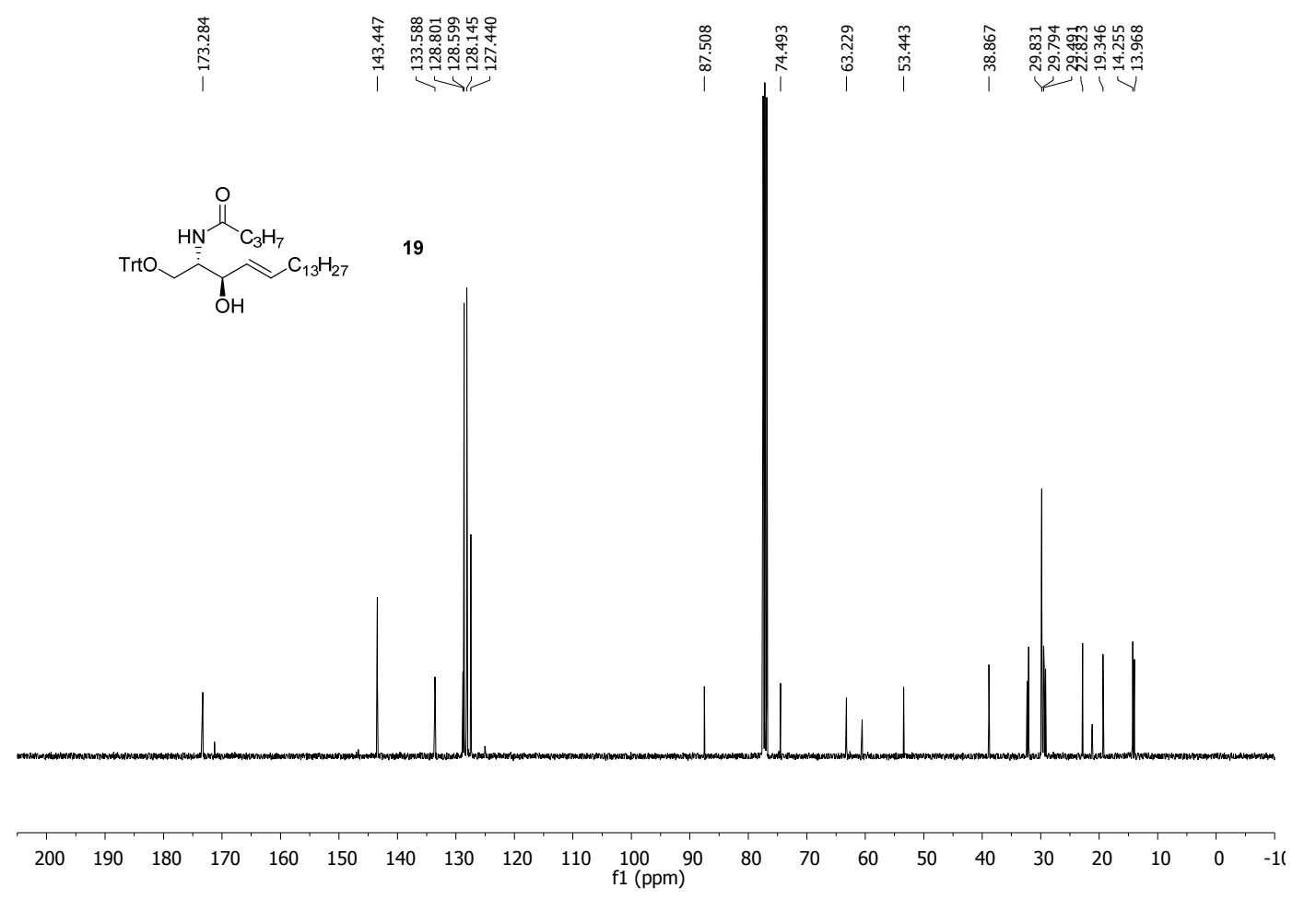

${ }^{13} \mathrm{C}$ NMR spectrum $\left(100.6 \mathrm{MHz}, \mathrm{CDCl}_{3}\right)$ of compound 19 (containing traces of pyridine and ethyl acetate). 


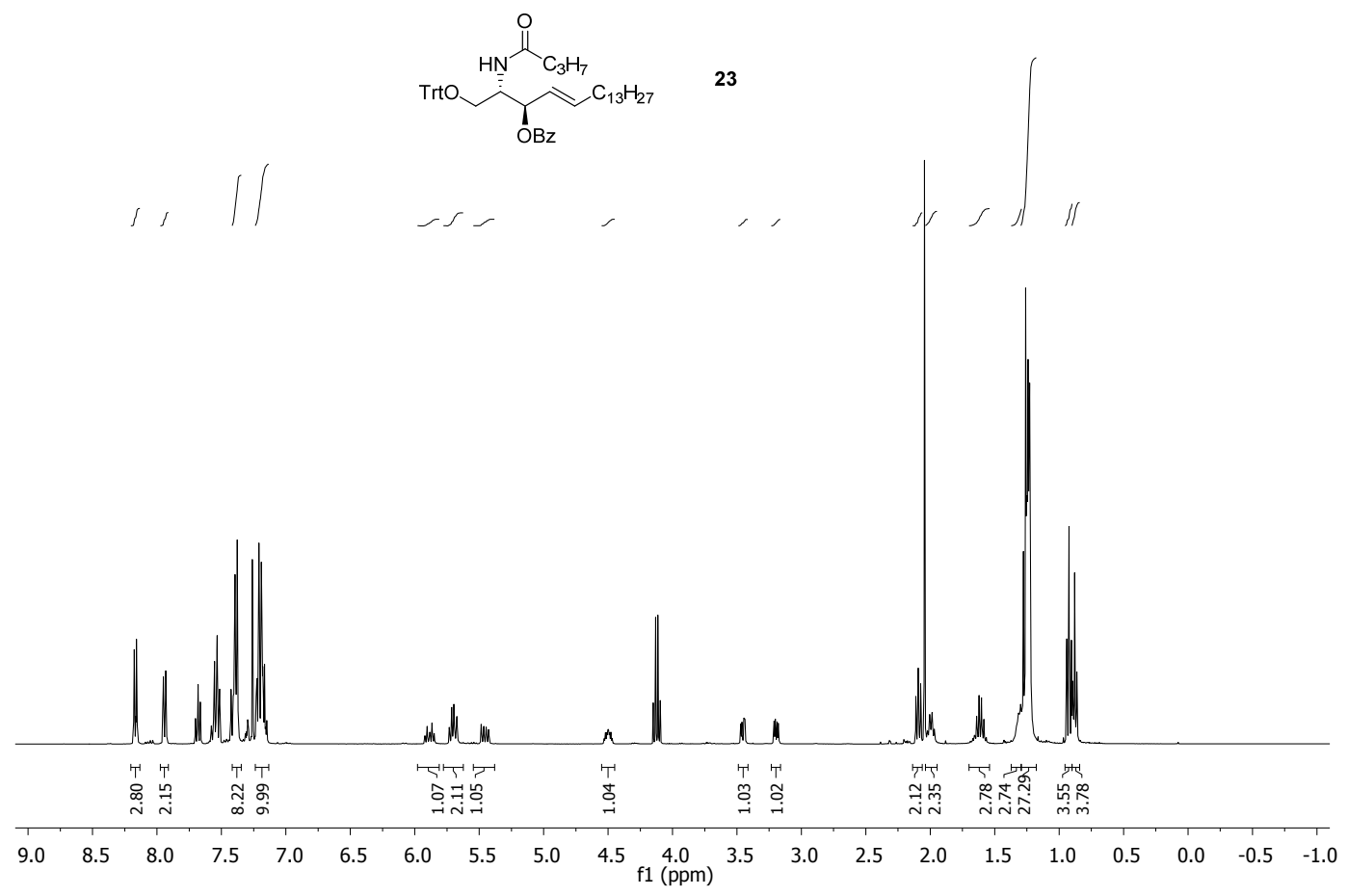

${ }^{1} \mathrm{H}$ NMR spectrum (400 MHz, $\mathrm{CDCl}_{3}$ ) of compound 23 (containing residual ethyl acetate).

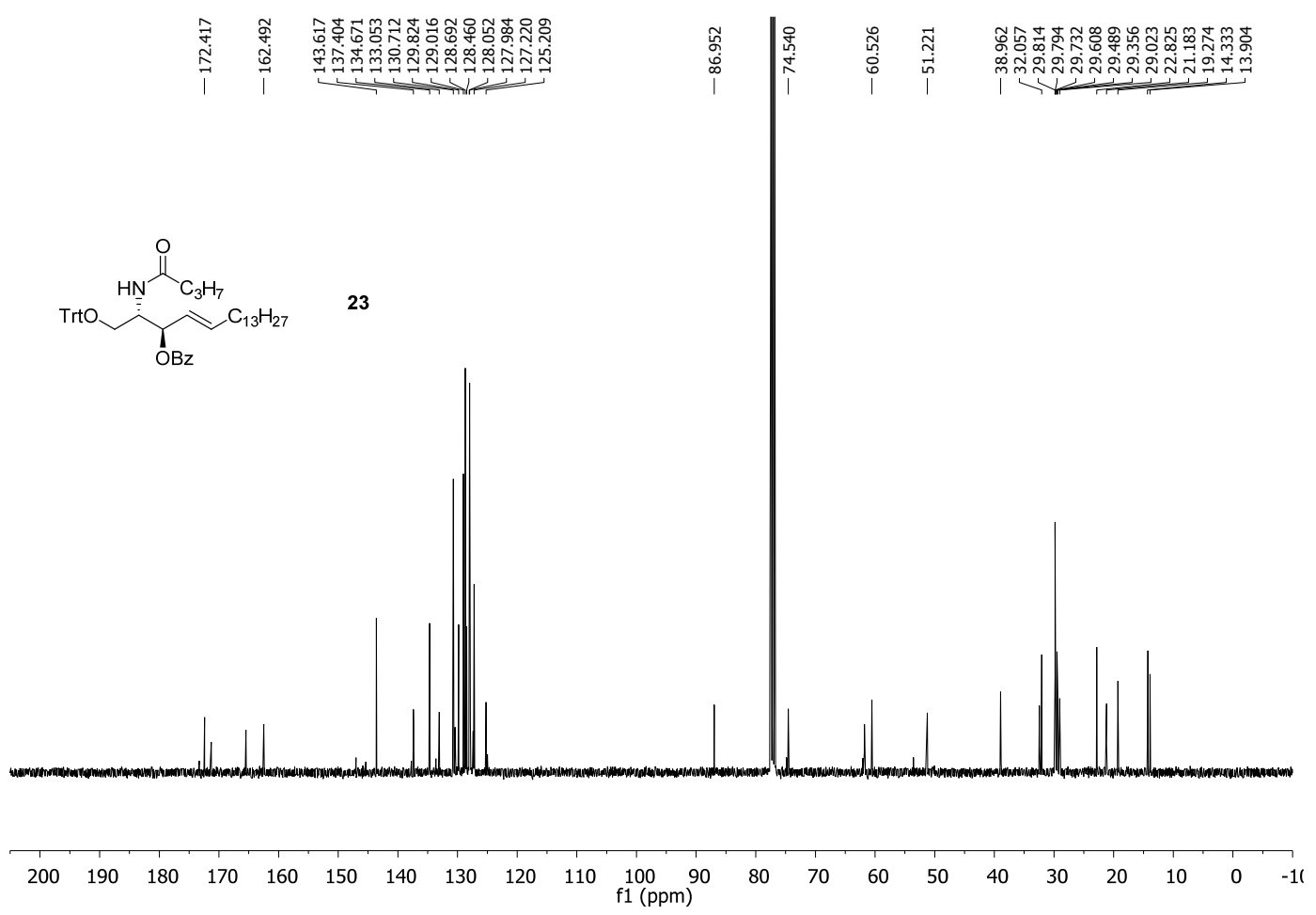

${ }^{13} \mathrm{C}$ NMR spectrum $\left(100.6 \mathrm{MHz}, \mathrm{CDCl}_{3}\right.$ ) of compound 23 (containing residual ethyl acetate). 


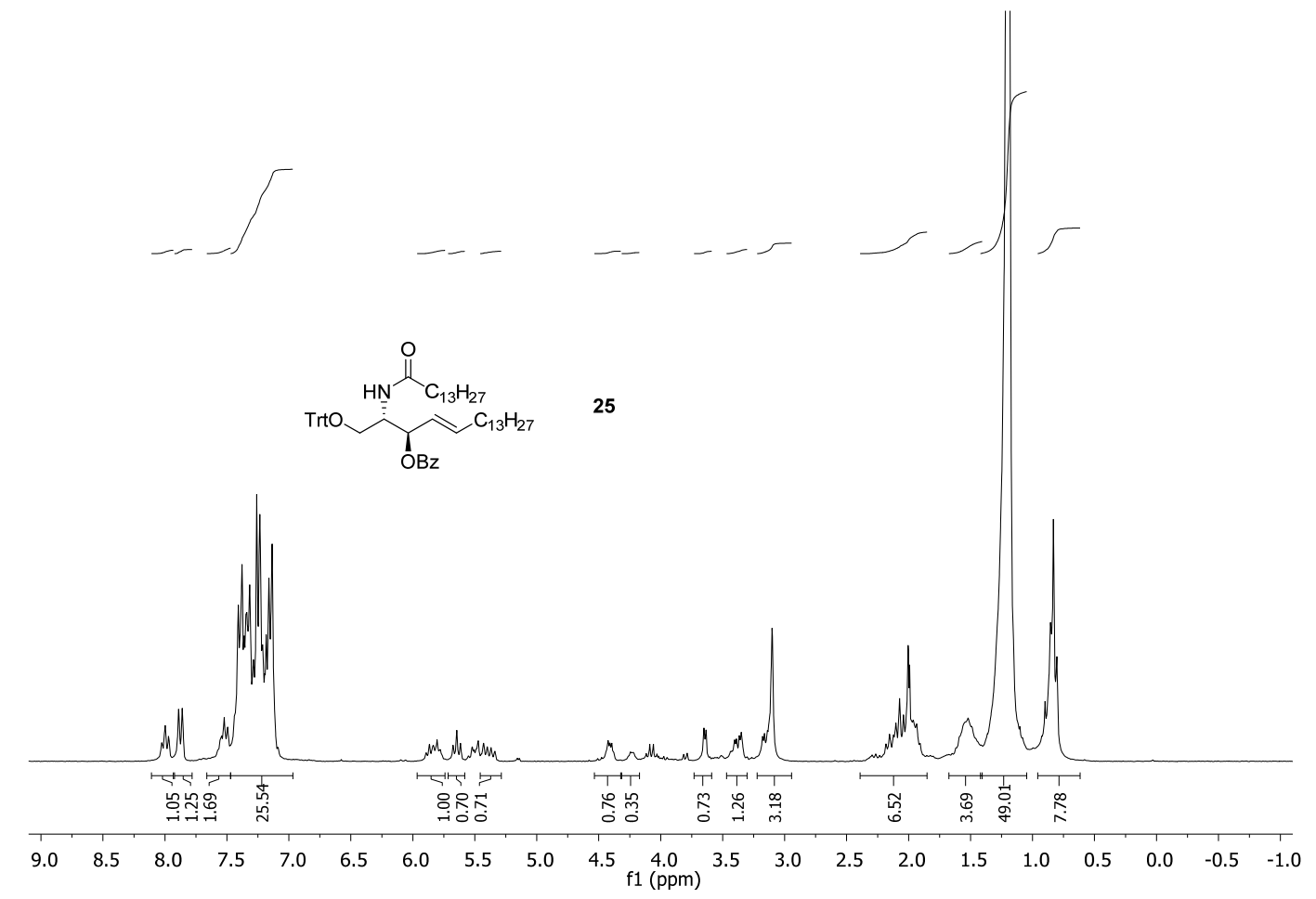

${ }^{1} \mathrm{H}$ NMR spectrum $\left(400 \mathrm{MHz}, \mathrm{CDCl}_{3}\right)$ of compound 25. 


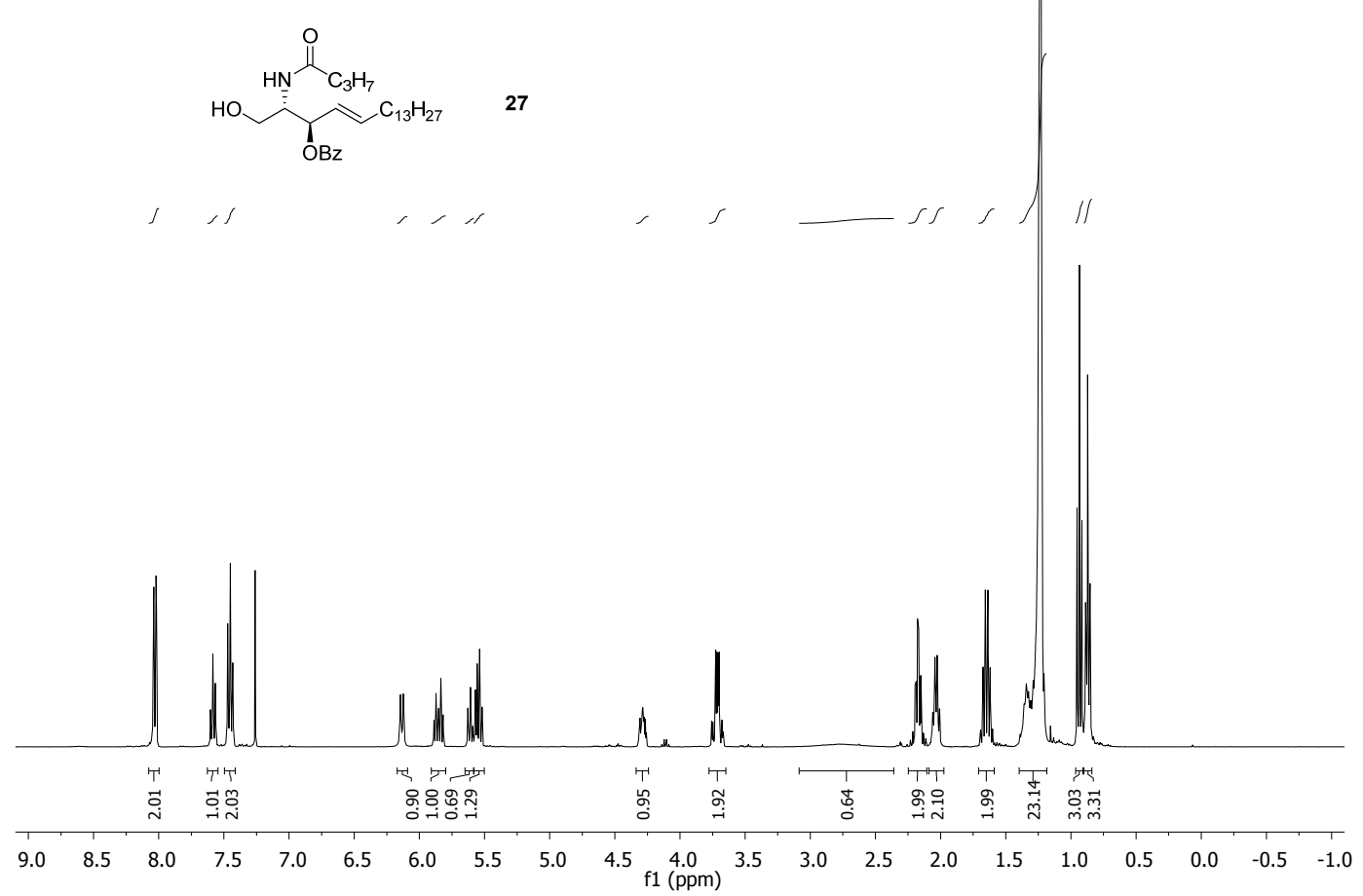

${ }^{1} \mathrm{H}$ NMR spectrum (400 MHz, $\mathrm{CDCl}_{3}$ ) of compound 27.
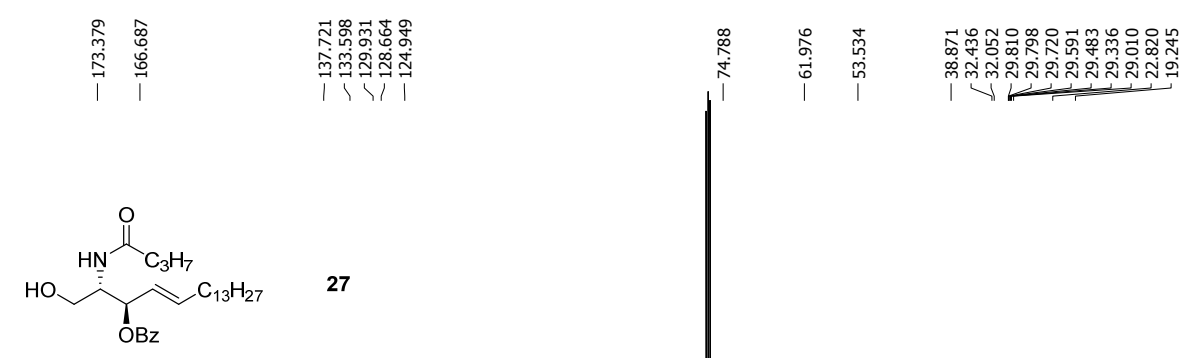

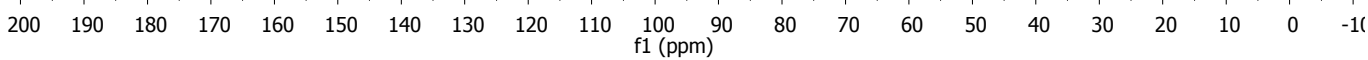

${ }^{13} \mathrm{C}$ NMR spectrum (100.6 MHz, $\mathrm{CDCl}_{3}$ ) of compound 27. 


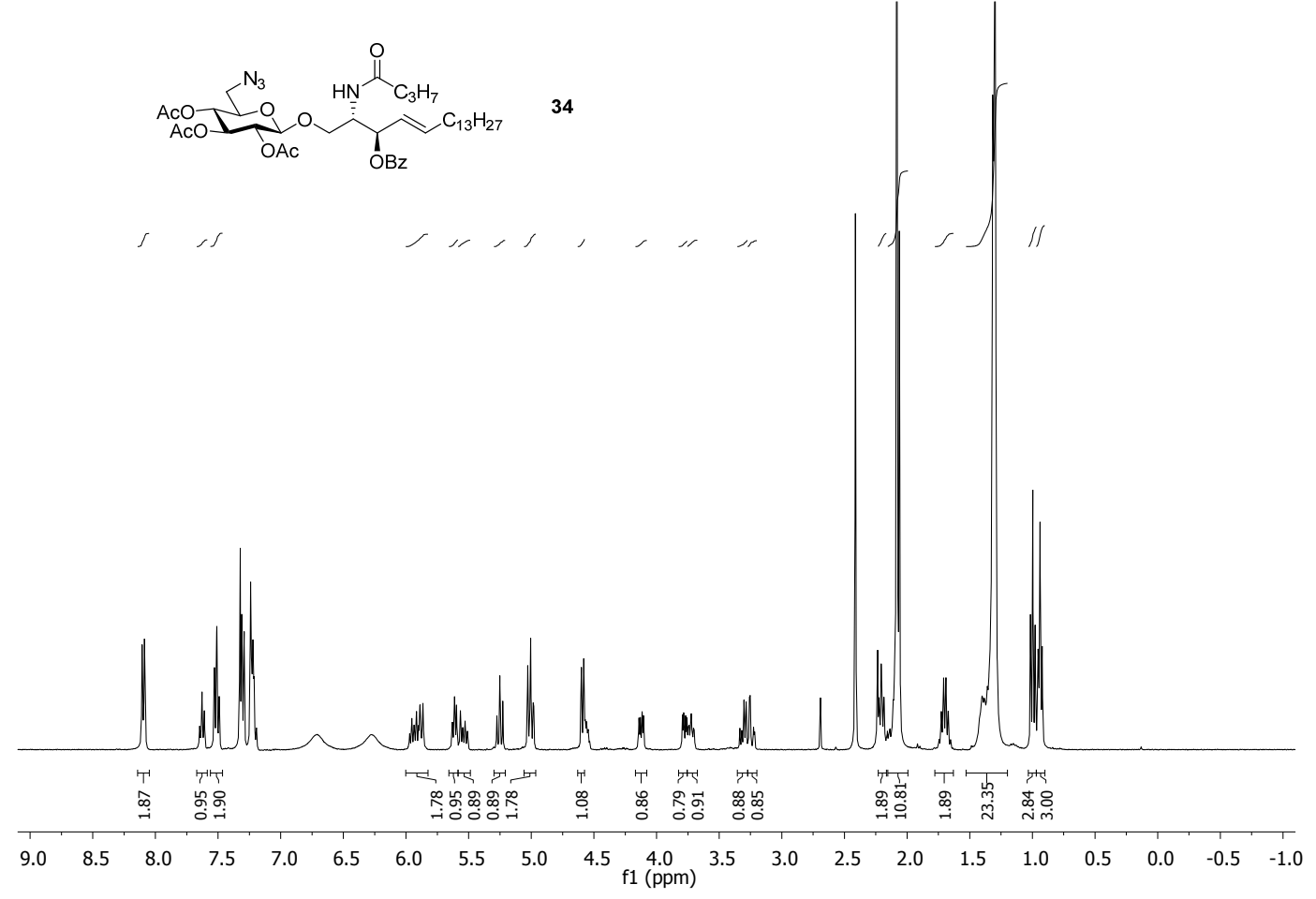

${ }^{1} \mathrm{H}$ NMR spectrum (400 MHz, $\mathrm{CDCl}_{3}$ ) of compound 34 (containing residual toluene and trichloroacetamide).

\begin{tabular}{|c|c|}
\hline 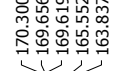 & 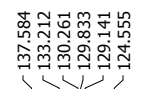 \\
\hline
\end{tabular}

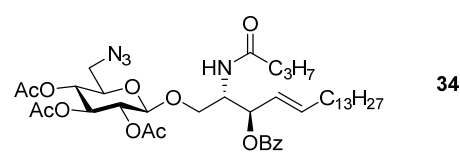

34
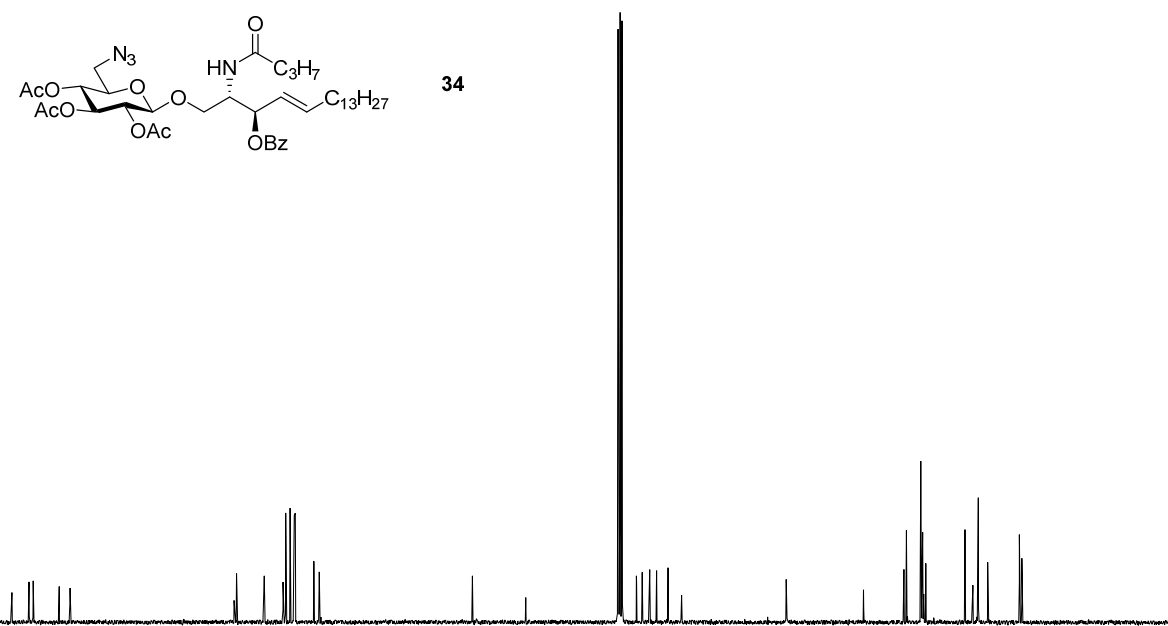

$\left.\begin{array}{lllllllllll}200 & 190 & 180 & 170 & 160 & 150 & 140 & 130 & 120 & 110 & 100 \\ \mathrm{f} 1(\mathrm{ppm})\end{array}\right)$

${ }^{13} \mathrm{C}$ NMR spectrum $\left(100.6 \mathrm{MHz}, \mathrm{CDCl}_{3}\right.$ ) of compound 34 (containing residual toluene and trichloroacetamide). 


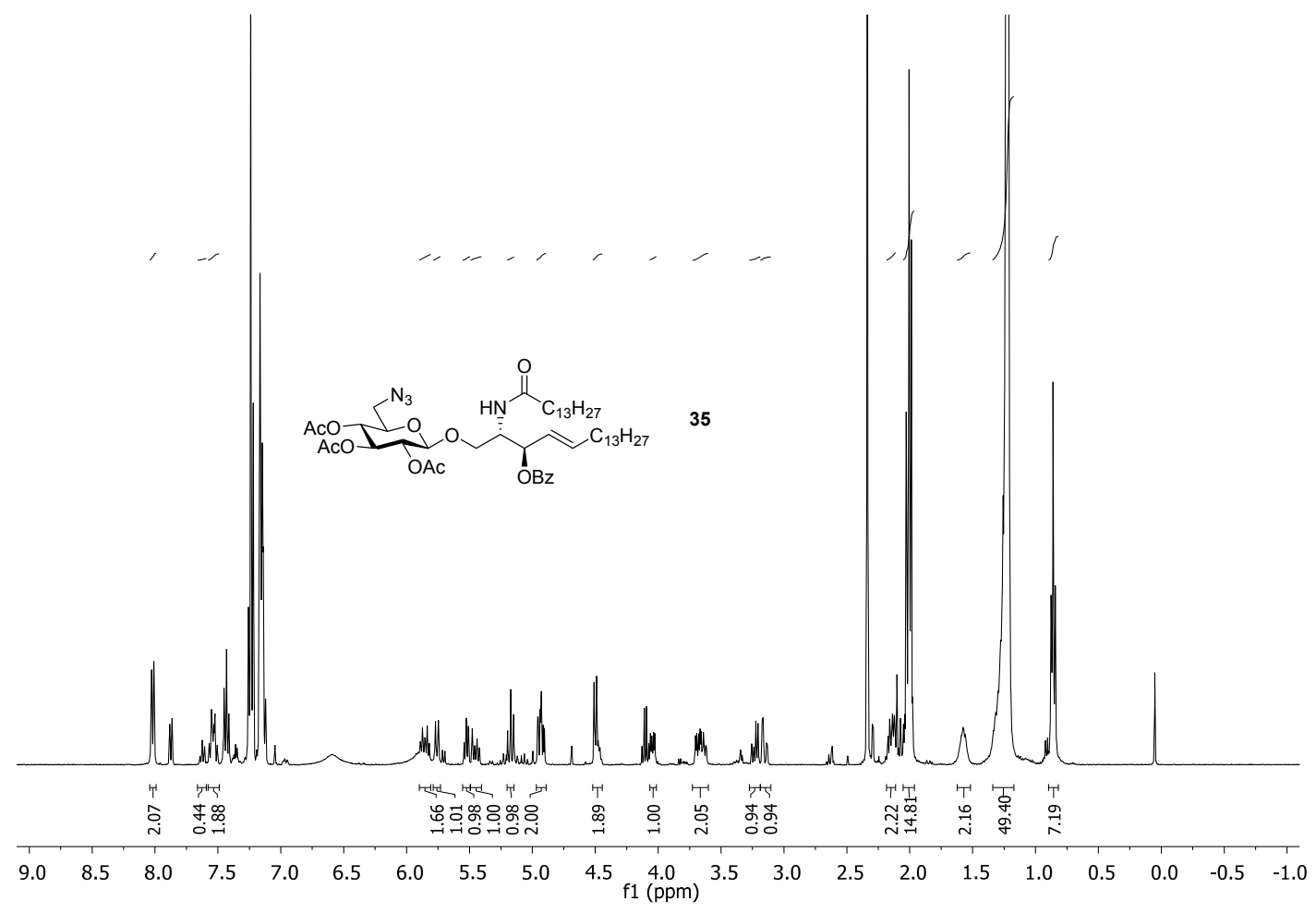

${ }^{1} \mathrm{H}$ NMR spectrum ( $400 \mathrm{MHz}, \mathrm{CDCl}_{3}$ ) of compound $\mathbf{3 5}$ (containing residual toluene and trichloroacetamide). 


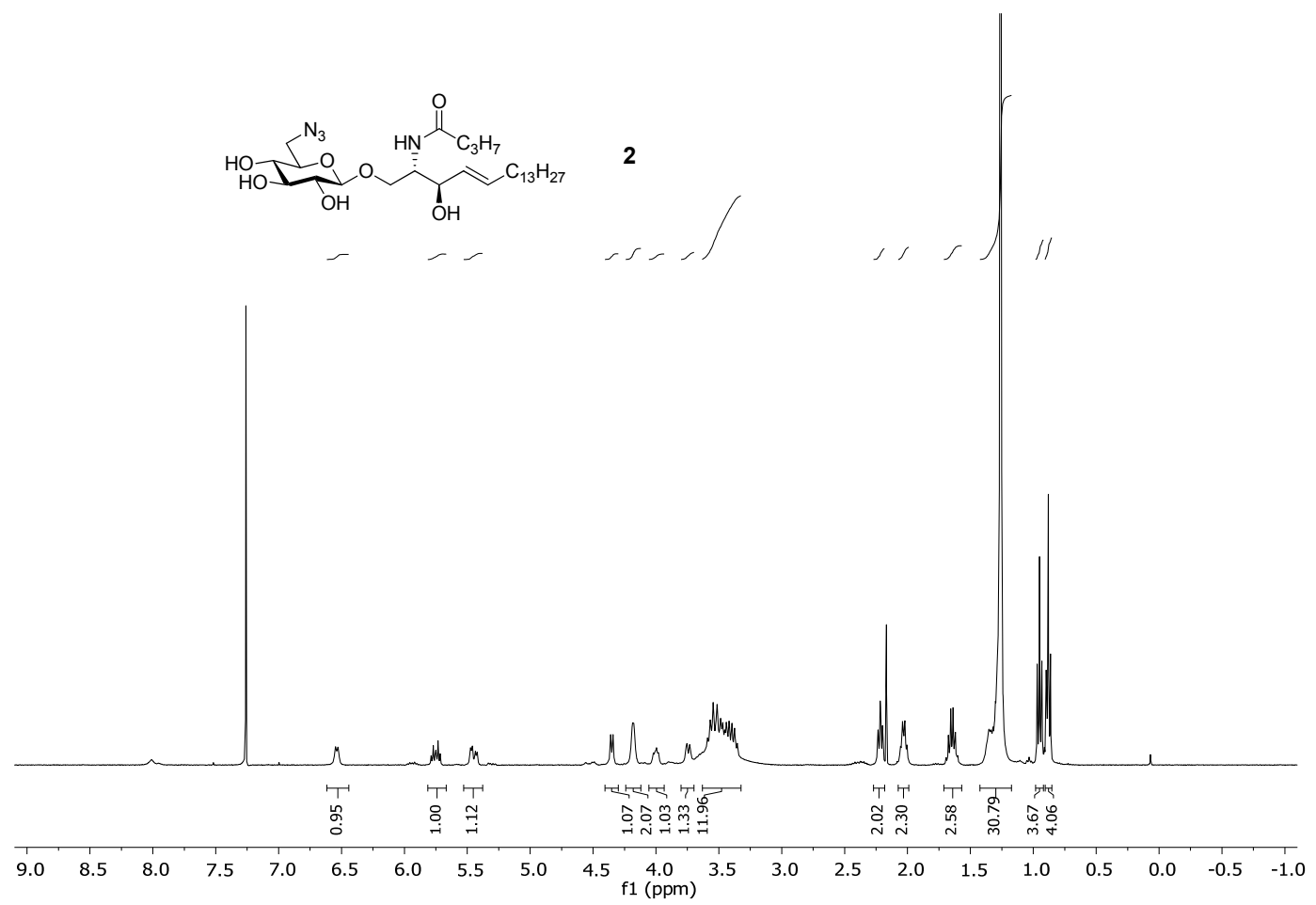

${ }^{1} \mathrm{H}$ NMR spectrum (400 MHz, $\mathrm{CDCl}_{3}$ ) of compound 2.

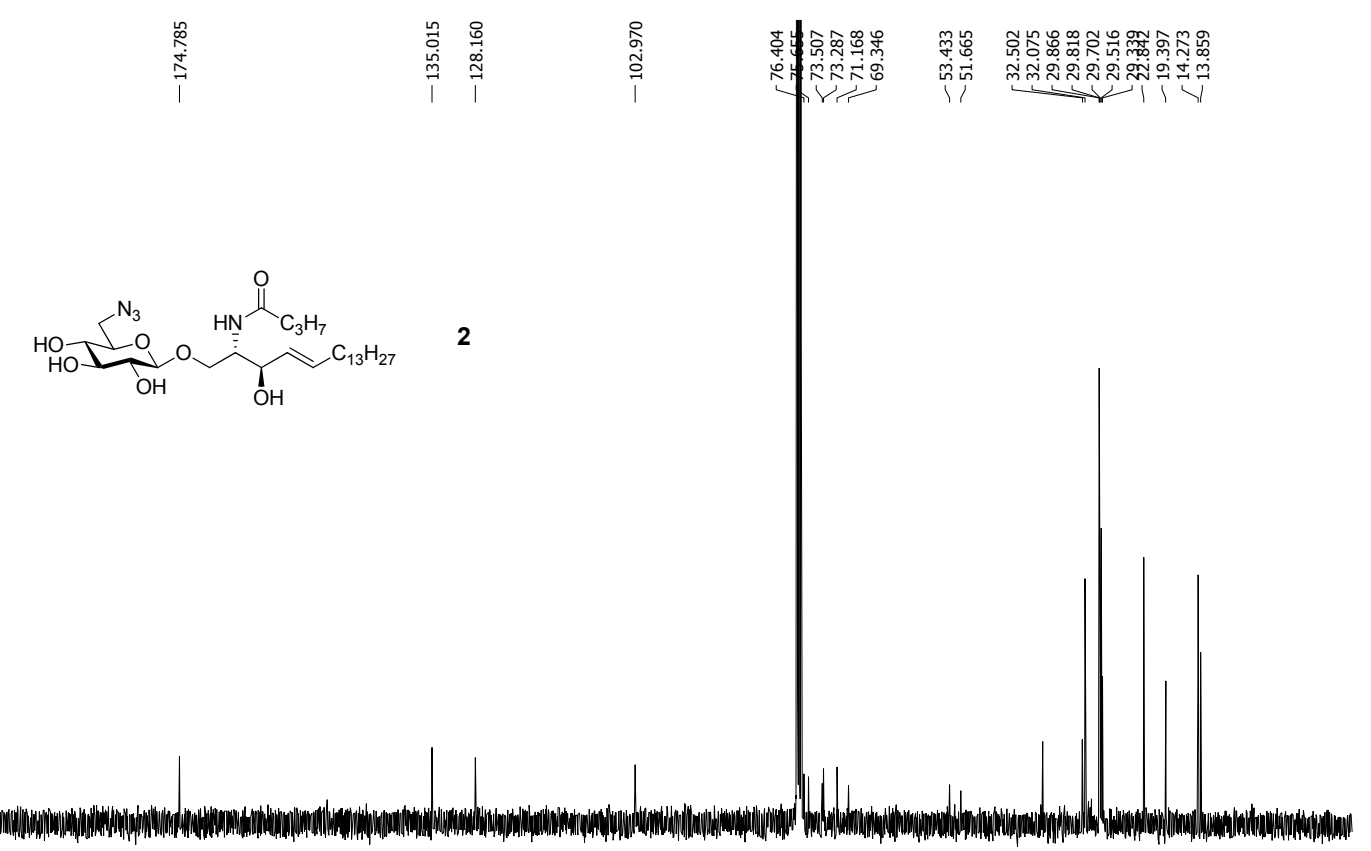

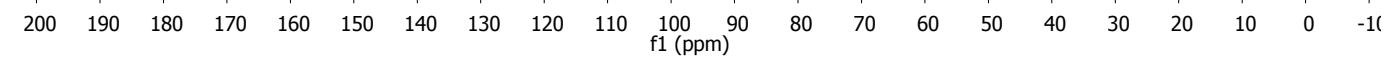

${ }^{13} \mathrm{C}$ NMR spectrum $\left(100.6 \mathrm{MHz}, \mathrm{CDCl}_{3}\right.$ ) of compound 2. 


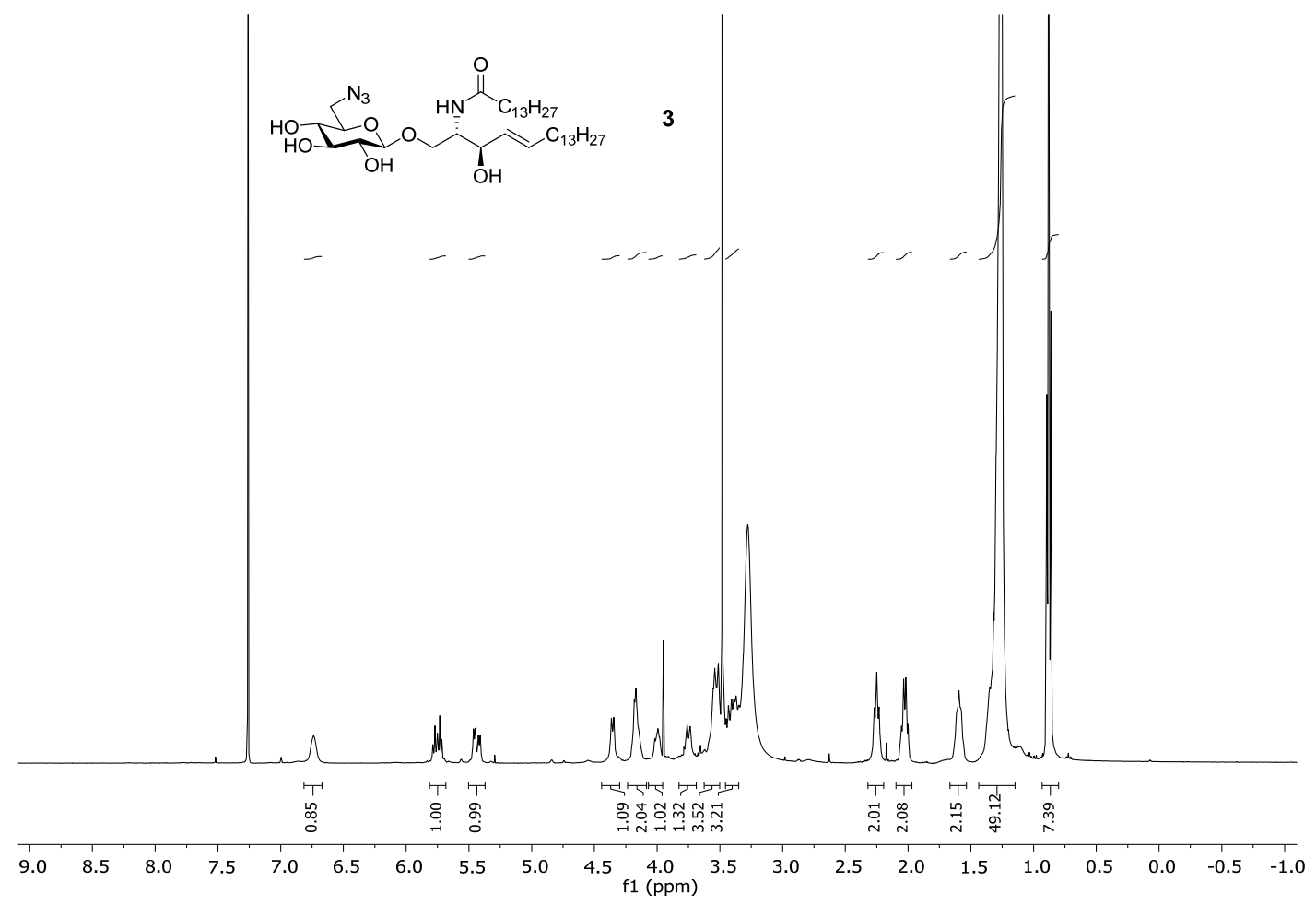

${ }^{1} \mathrm{H}$ NMR spectrum (400 MHz, $\mathrm{CDCl}_{3}$ ) of compound 3.

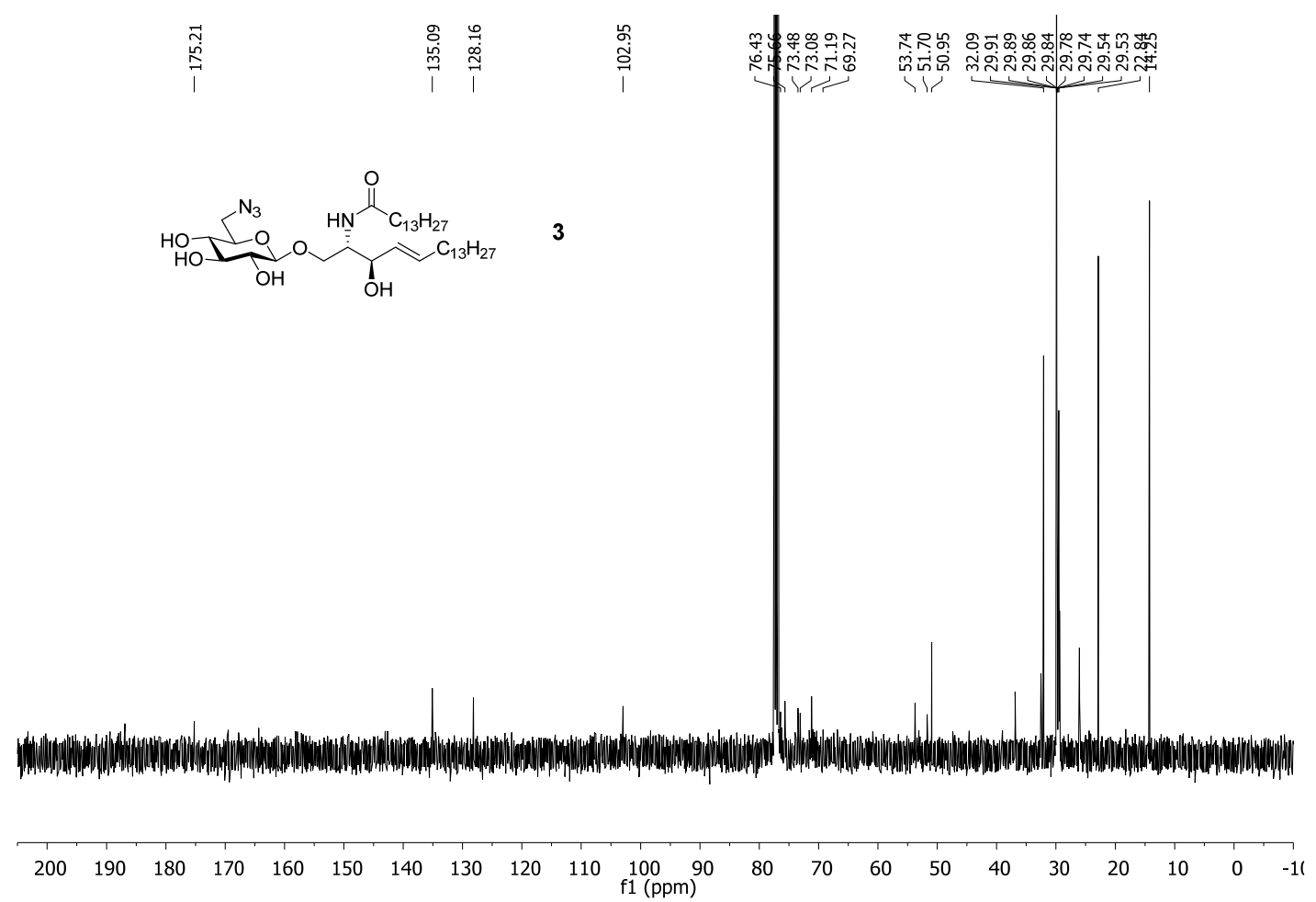

${ }^{13} \mathrm{C}$ NMR spectrum $\left(100.6 \mathrm{MHz}, \mathrm{CDCl}_{3}\right)$ of compound 3 . 


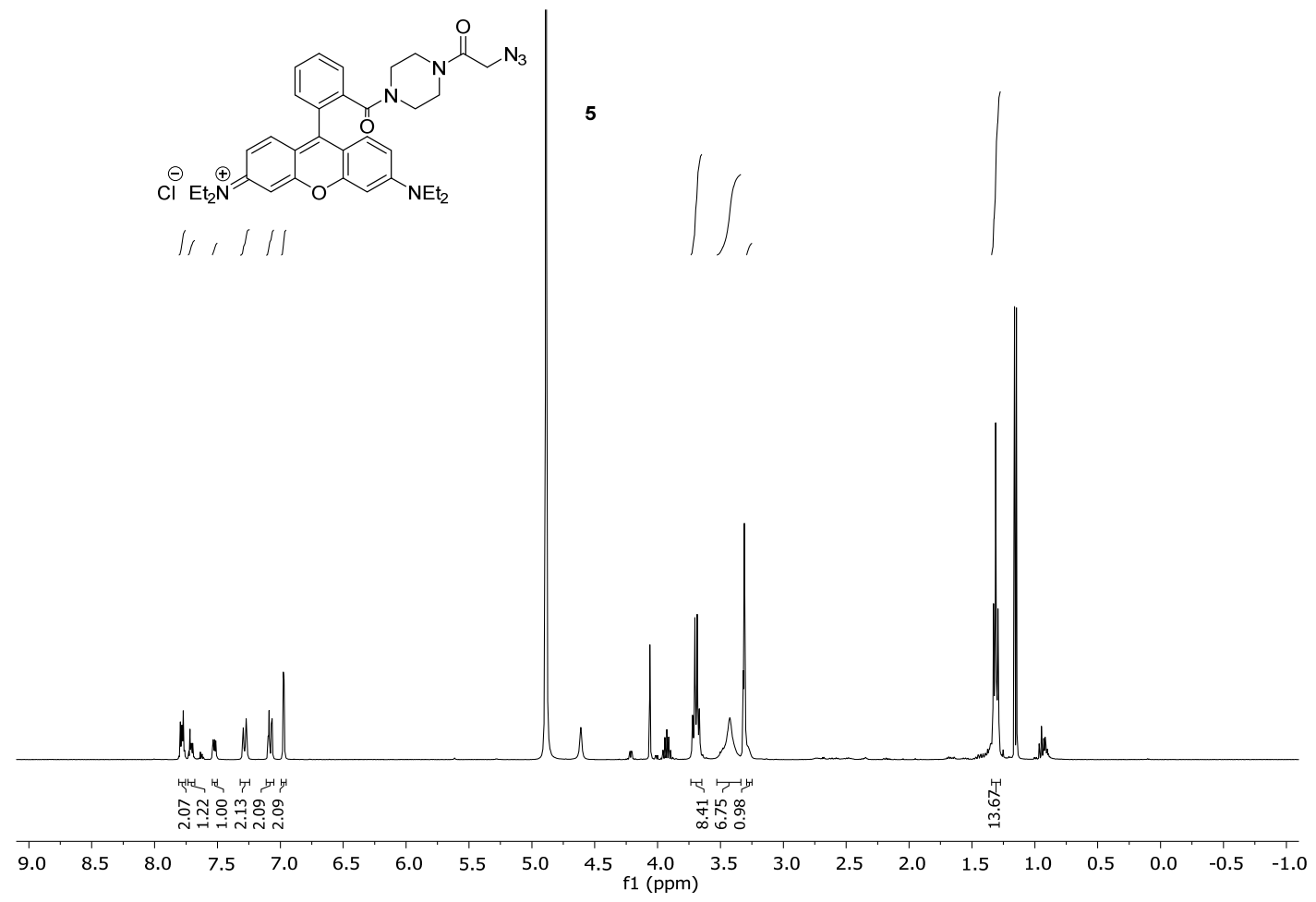

${ }^{1} \mathrm{H}$ NMR spectrum (400 MHz, $\mathrm{CD}_{3} \mathrm{OD}$ ) of compound 5.

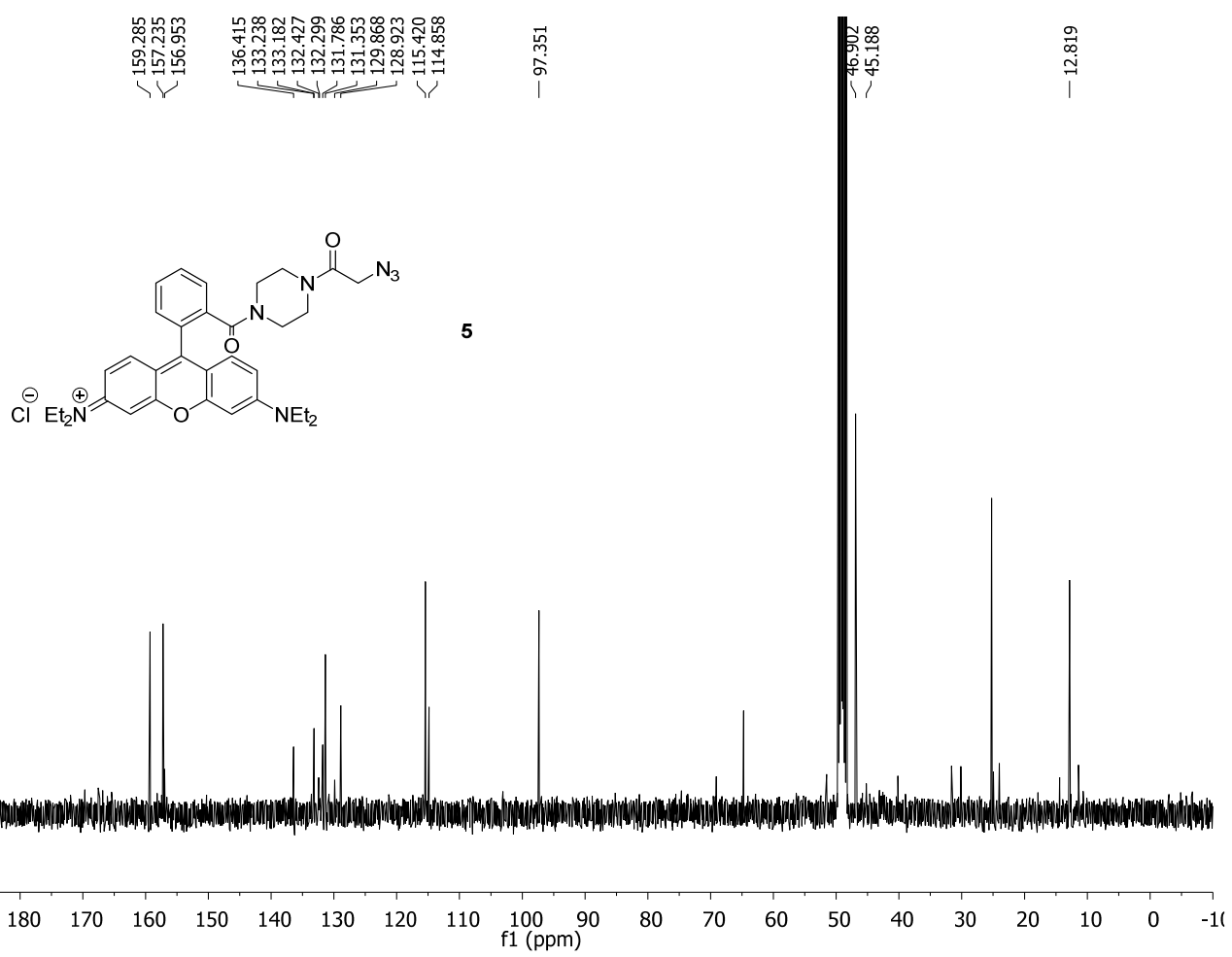

${ }^{13} \mathrm{C}$ NMR spectrum (100.6 MHz, $\left.\mathrm{CD}_{3} \mathrm{OD}\right)$ of compound 5. 


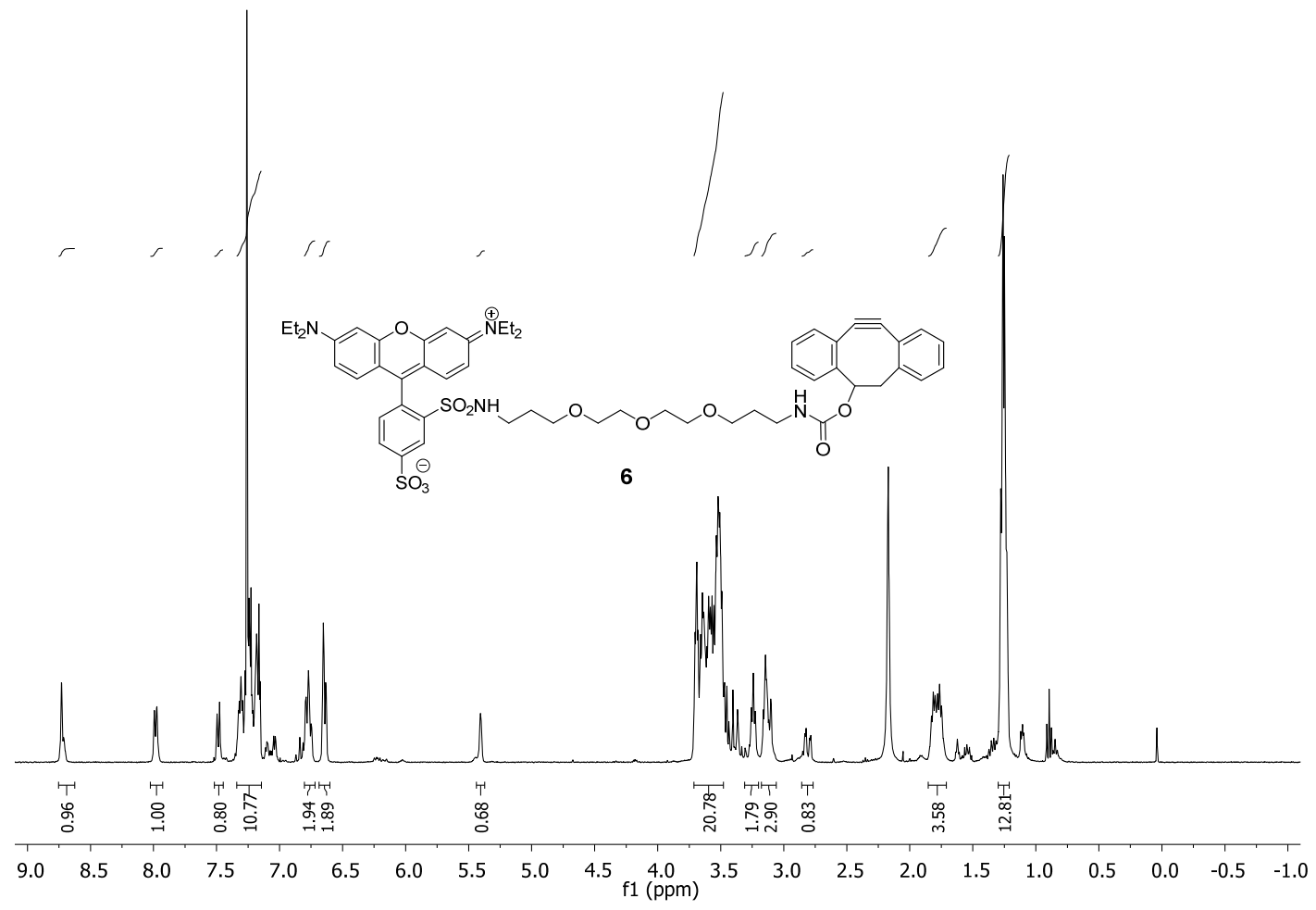

${ }^{1} \mathrm{H}$ NMR spectrum (400 MHz, $\mathrm{CDCl}_{3}$ ) of compound 6.

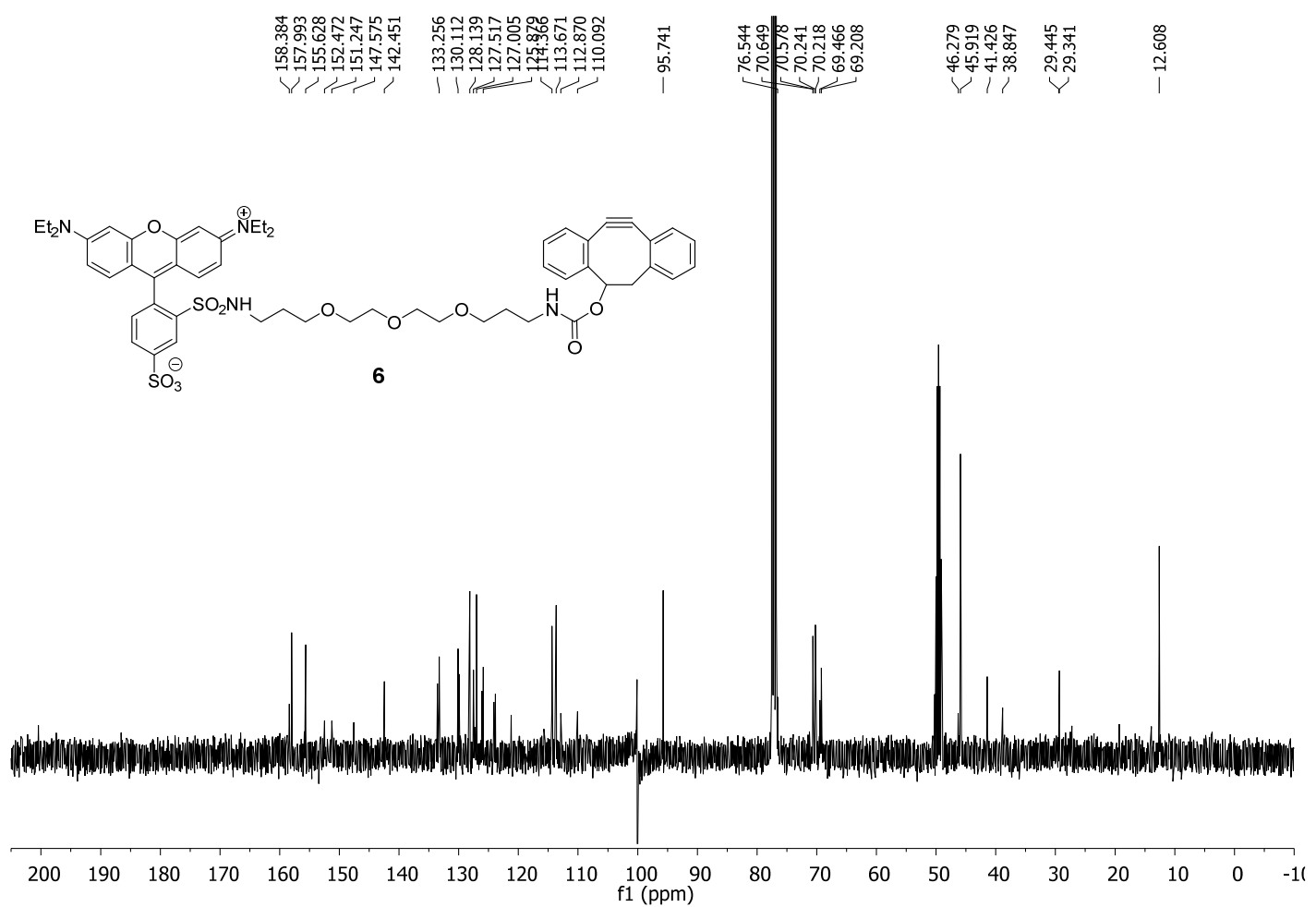

${ }^{13} \mathrm{C}$ NMR spectrum $\left(100.6 \mathrm{MHz}, \mathrm{CDCl}_{3}\right)$ of compound $\mathbf{6}$. 


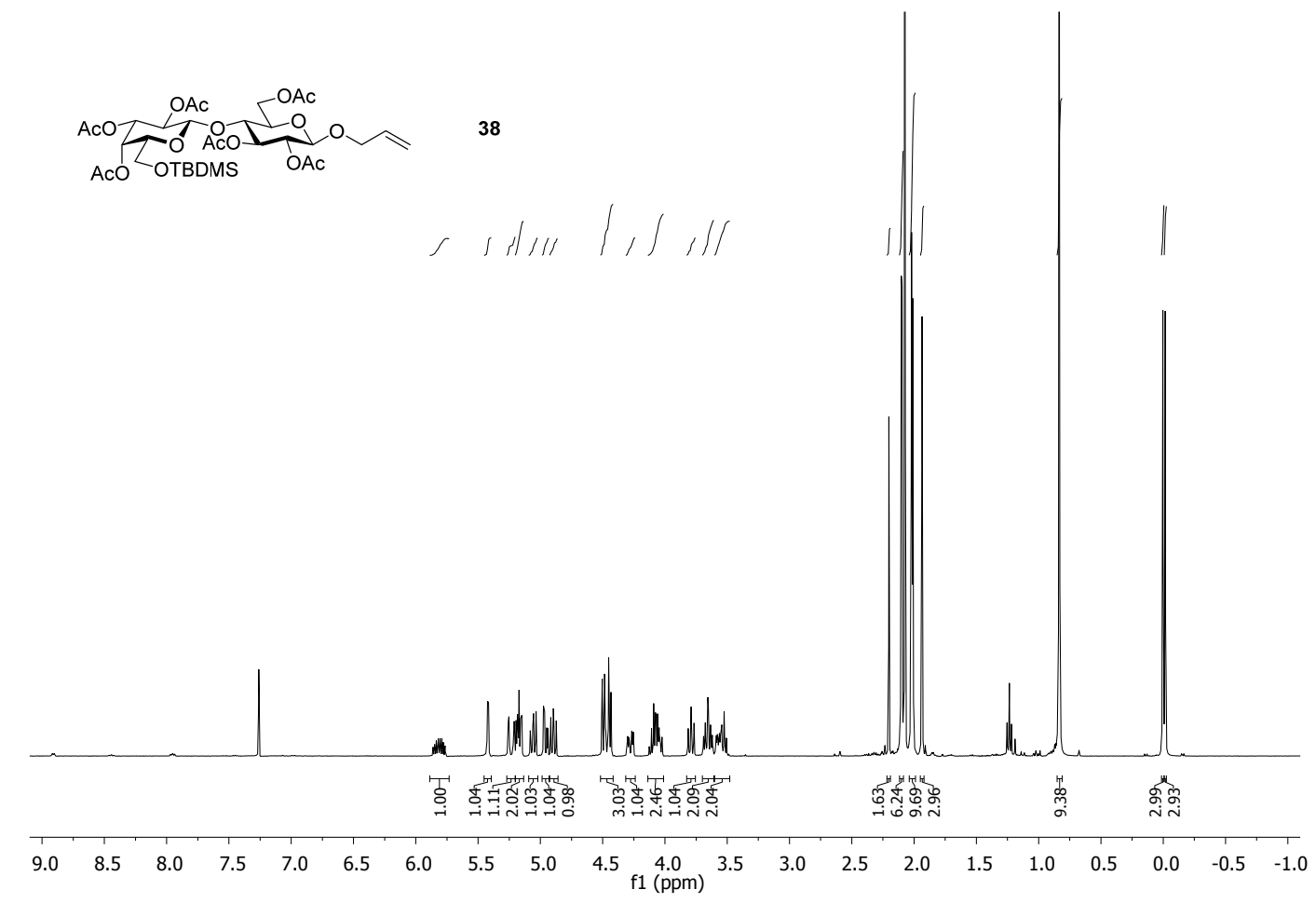

${ }^{1} \mathrm{H}$ NMR spectrum (400 MHz, $\mathrm{CDCl}_{3}$ ) of compound $\mathbf{3 8}$ (containing traces of ethyl acetate).

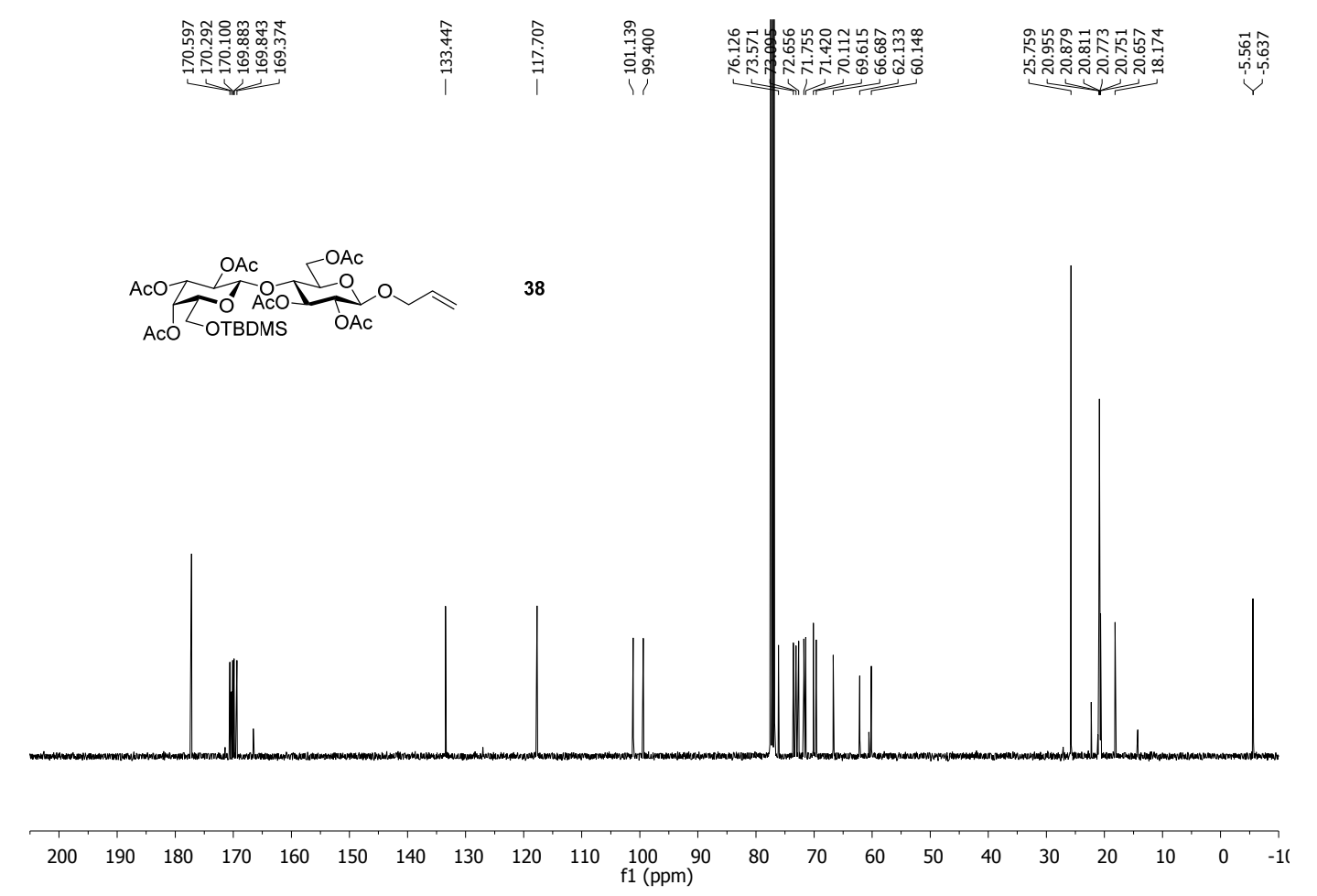

${ }^{13} \mathrm{C}$ NMR spectrum $\left(100.6 \mathrm{MHz}, \mathrm{CDCl}_{3}\right)$ of compound $\mathbf{3 8}$ (containing traces of ethyl acetate). 


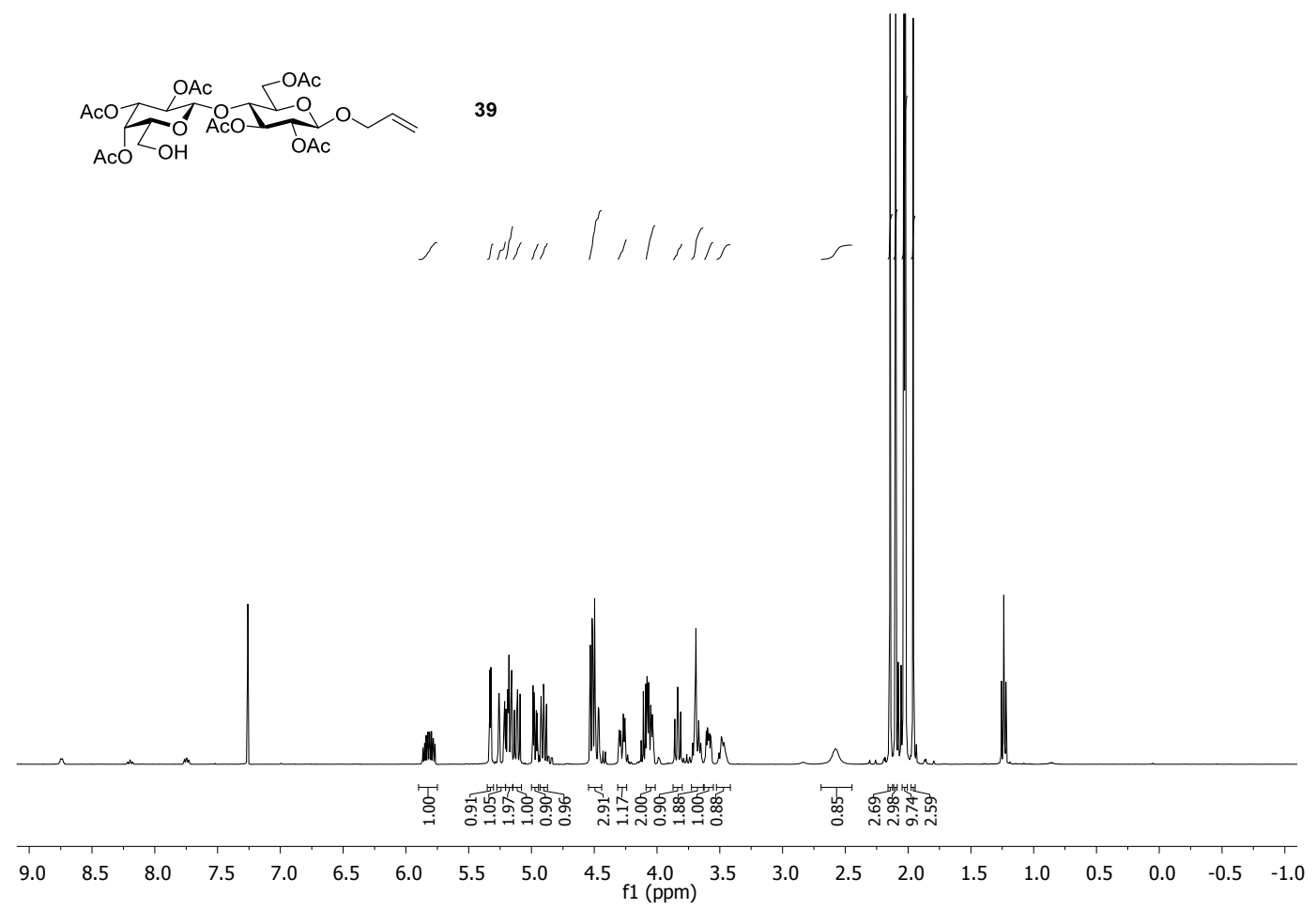

${ }^{1} \mathrm{H}$ NMR spectrum (400 MHz, $\mathrm{CDCl}_{3}$ ) of compound 39 (containing traces of pyridine and ethyl acetate).

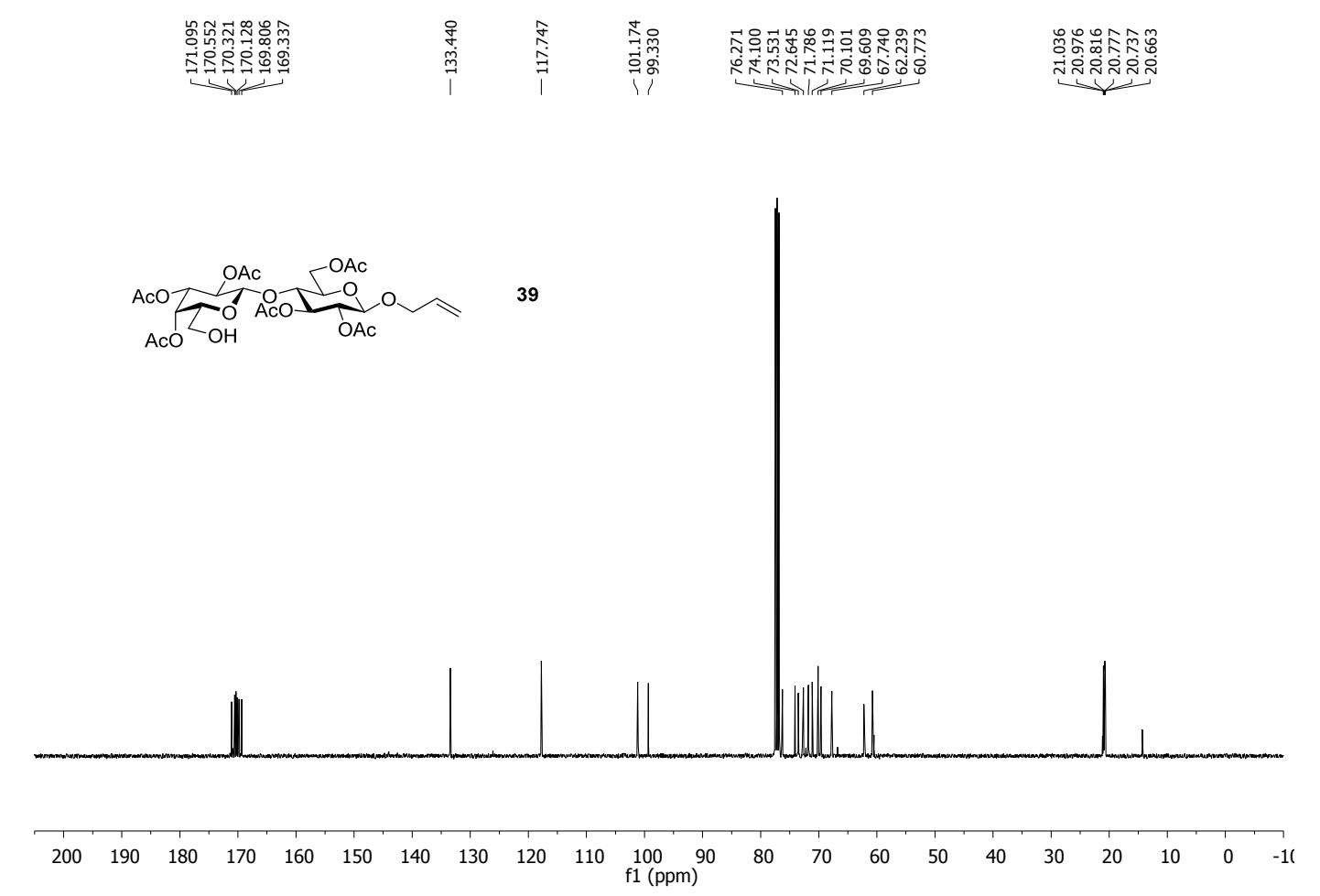

${ }^{13} \mathrm{C}$ NMR spectrum $\left(100.6 \mathrm{MHz}, \mathrm{CDCl}_{3}\right.$ ) of compound $\mathbf{3 9}$ (containing traces of pyridine and ethyl acetate). 


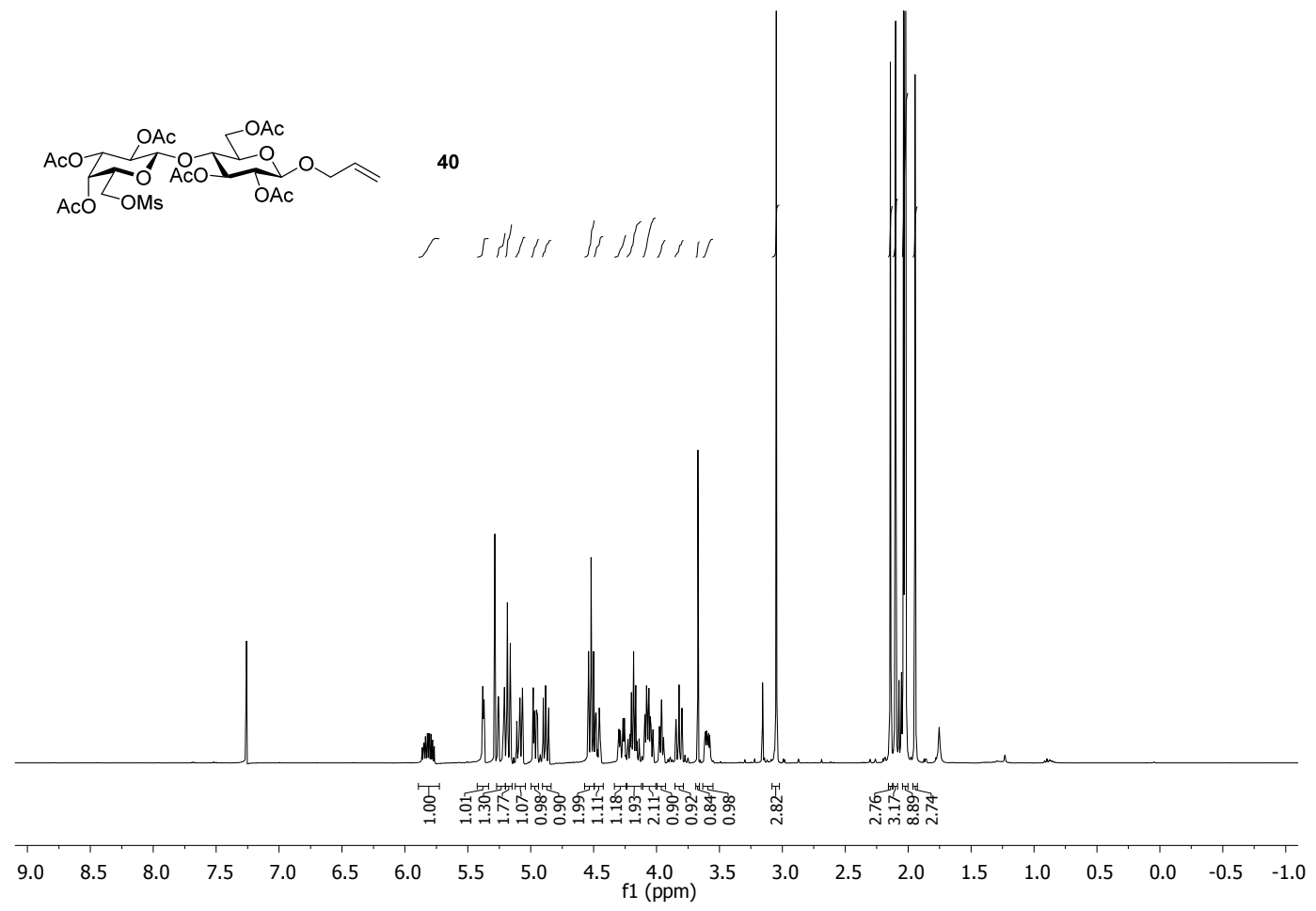

${ }^{1} \mathrm{H}$ NMR spectrum $\left(400 \mathrm{MHz}, \mathrm{CDCl}_{3}\right)$ of compound $\mathbf{4 0}$.

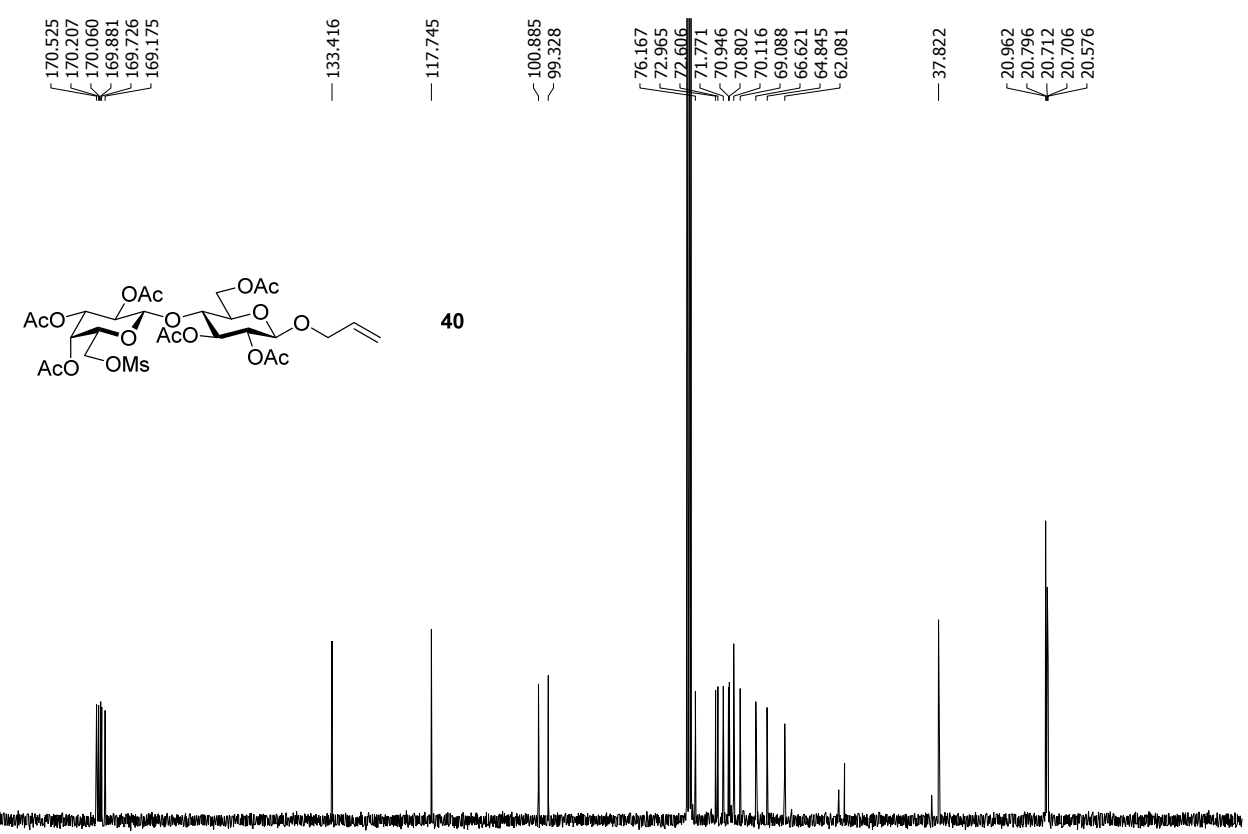

$\begin{array}{llllllllllllllllllllll}200 & 190 & 180 & 170 & 160 & 150 & 140 & 130 & 120 & 110 & \begin{array}{c}100 \\ \mathrm{f} 1(\mathrm{ppm})\end{array} & 90 & 80 & 70 & 60 & 50 & 40 & 30 & 20 & 10 & 0 & -1(\end{array}$

${ }^{13} \mathrm{C}$ NMR spectrum $\left(100.6 \mathrm{MHz}, \mathrm{CDCl}_{3}\right)$ of compound $\mathbf{4 0}$. 


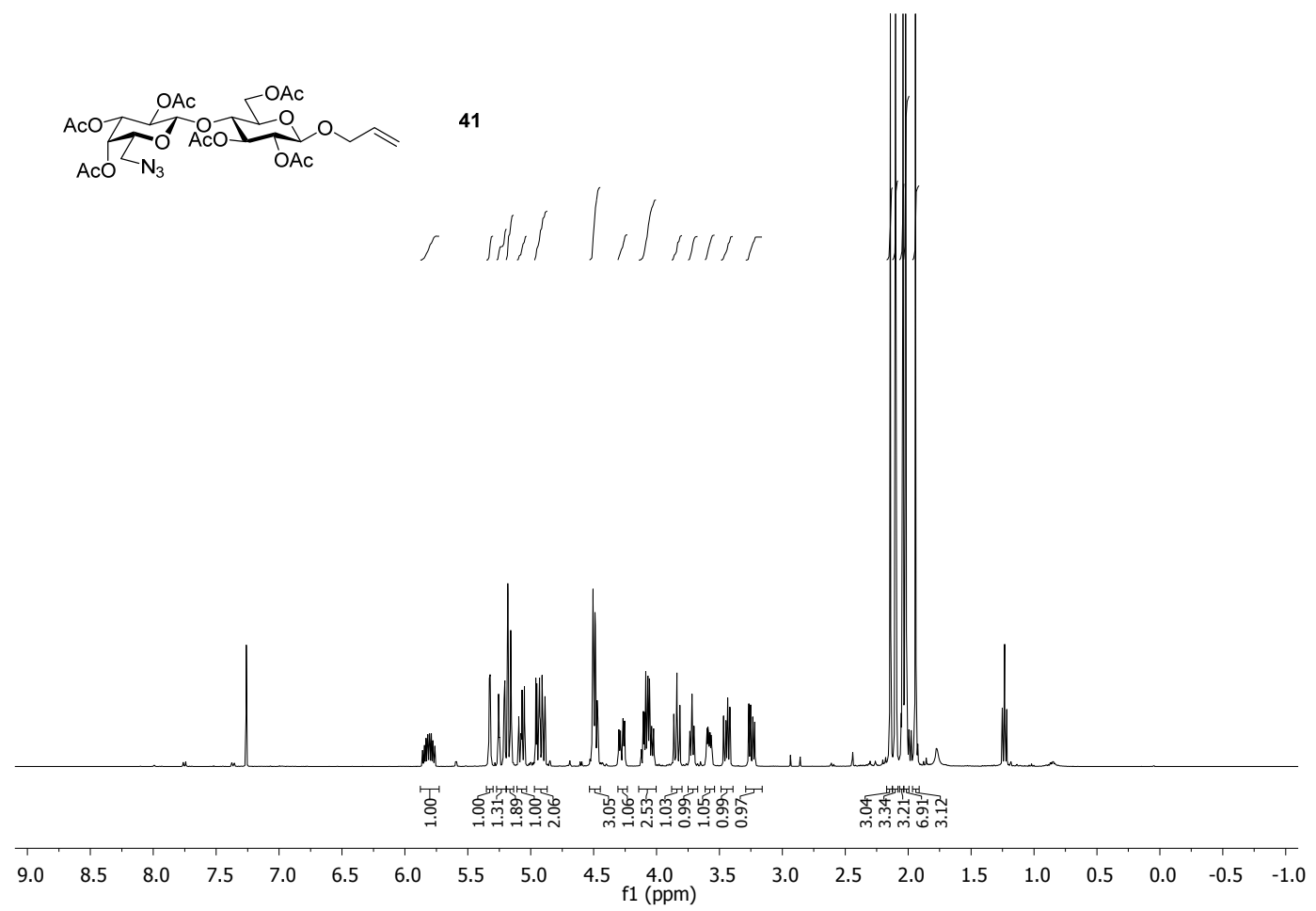

${ }^{1} \mathrm{H}$ NMR spectrum (400 MHz, $\mathrm{CDCl}_{3}$ ) of compound 41 (containing residual ethyl acetate).

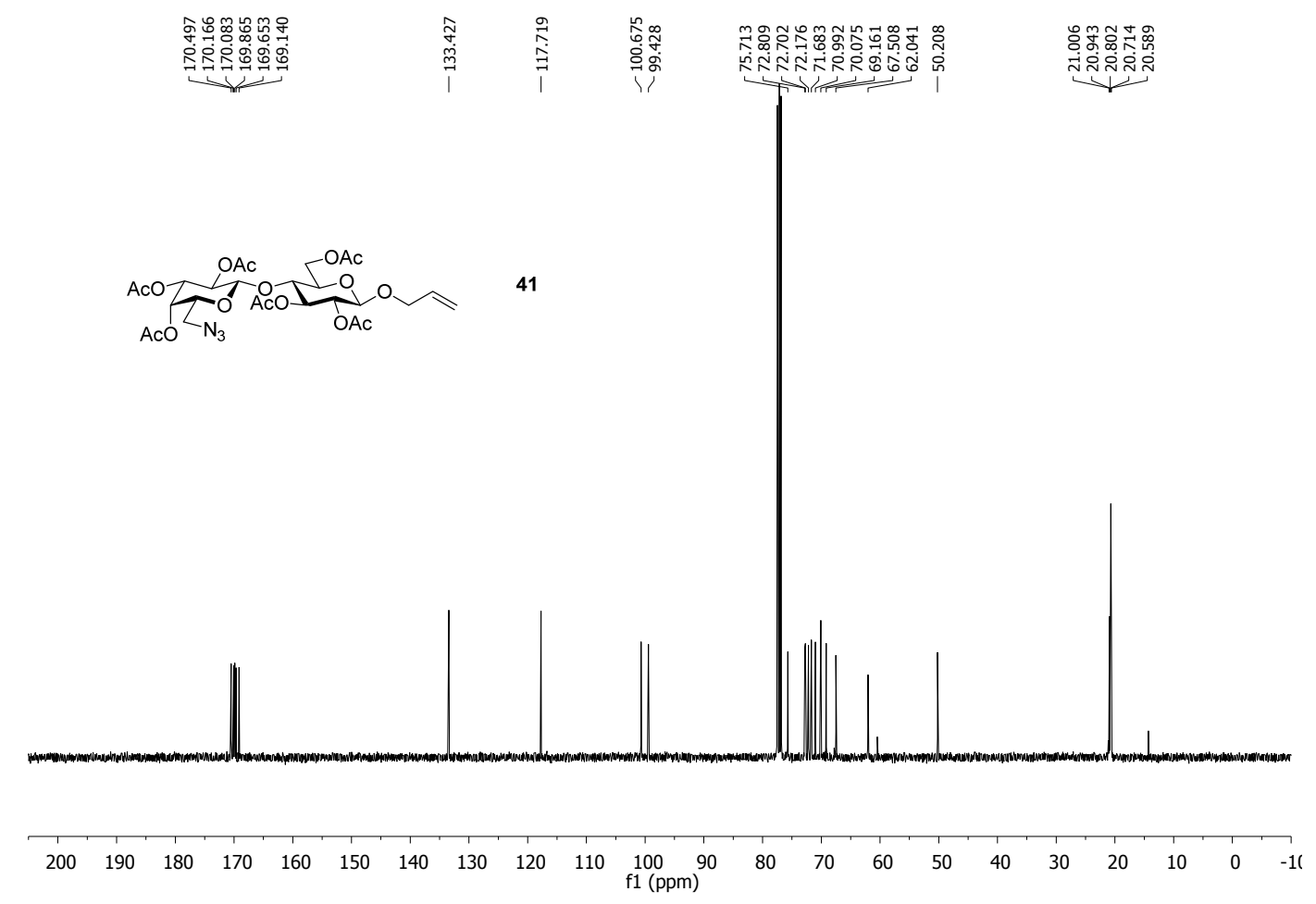

${ }^{13} \mathrm{C}$ NMR spectrum $\left(100.6 \mathrm{MHz}, \mathrm{CDCl}_{3}\right.$ ) of compound 41 (containing residual ethyl acetate). 


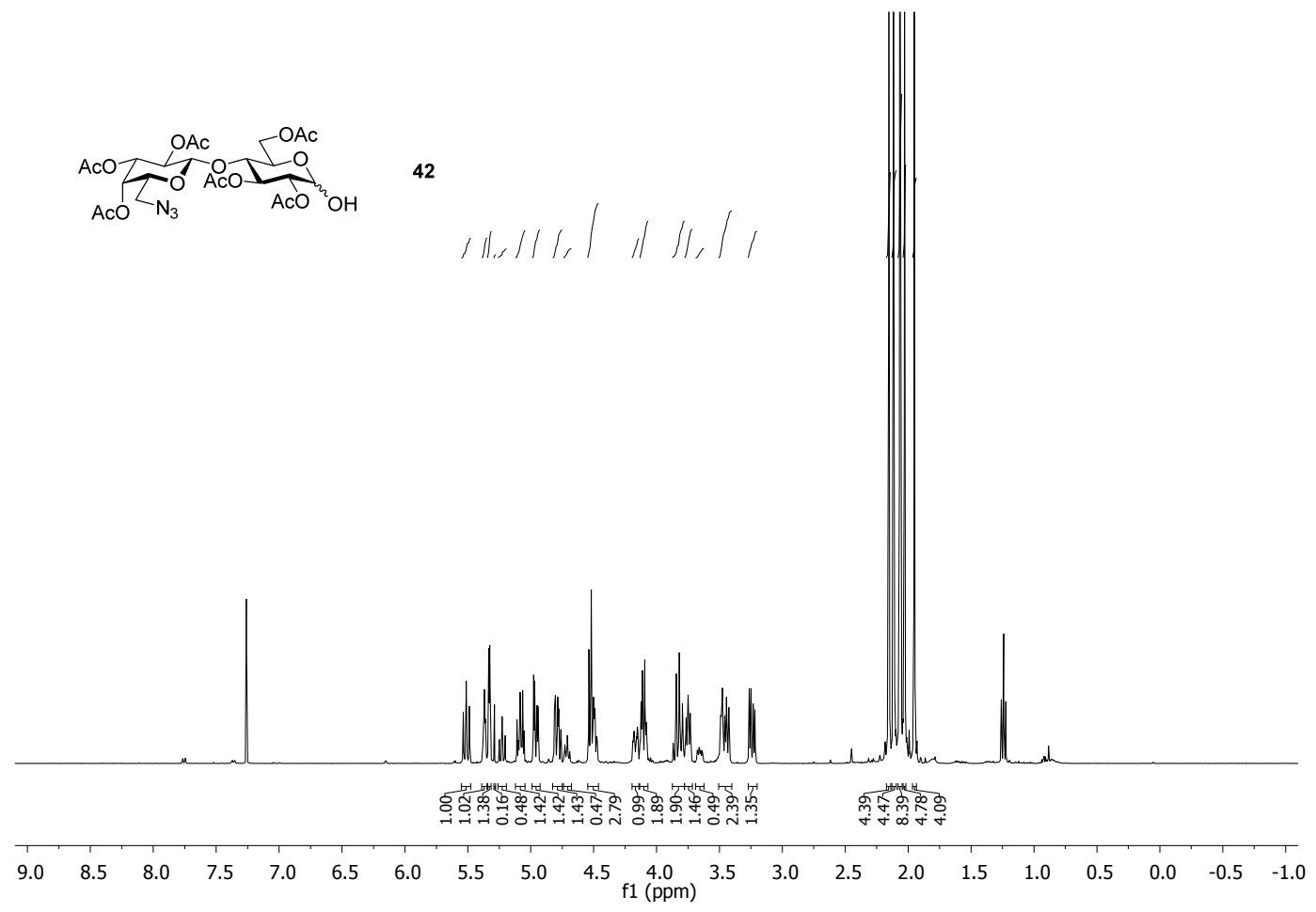

${ }^{1} \mathrm{H}$ NMR spectrum (400 MHz, $\mathrm{CDCl}_{3}$ ) of compound 42 (containing residual ethyl acetate).

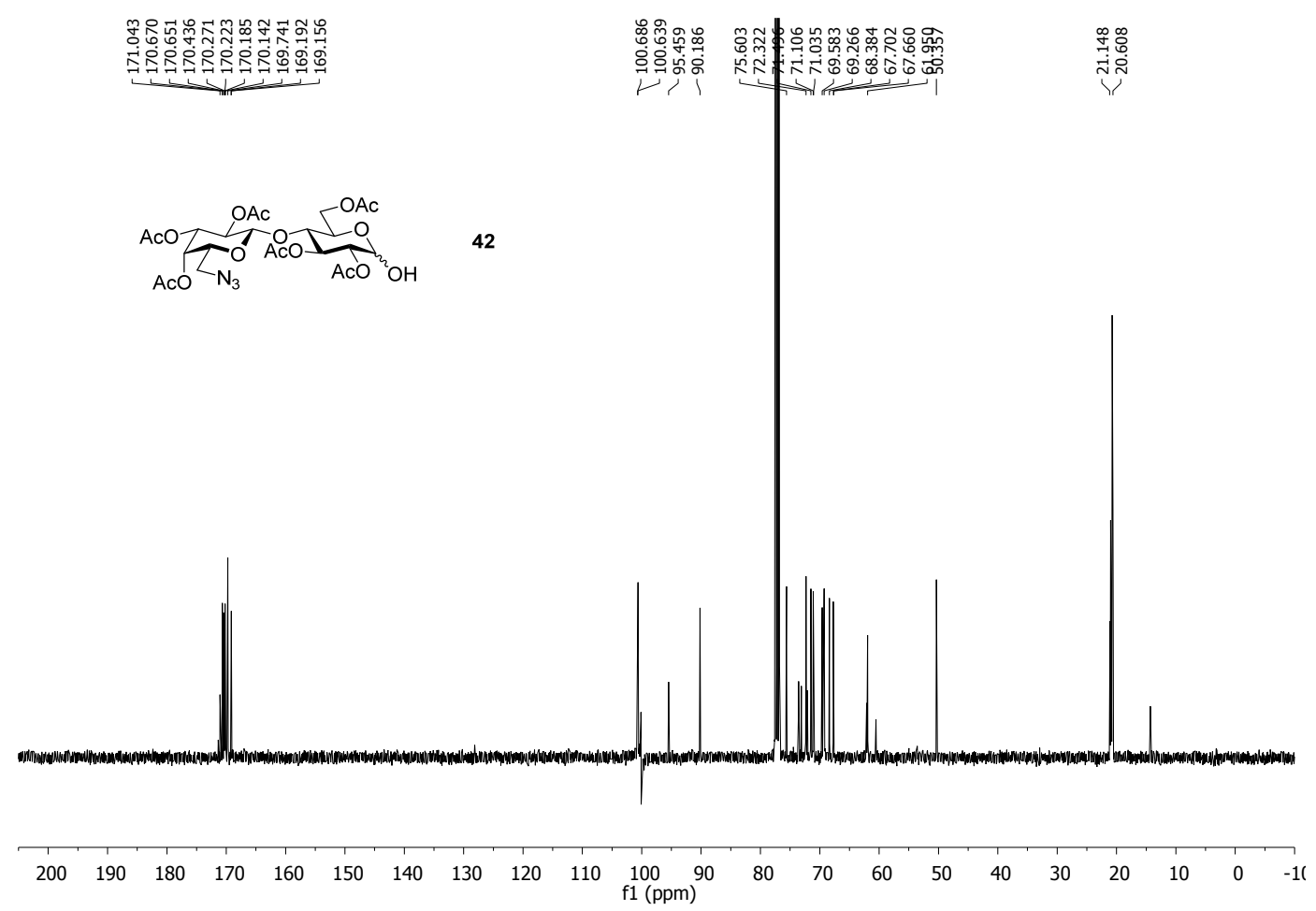

${ }^{13} \mathrm{C}$ NMR spectrum $\left(100.6 \mathrm{MHz}, \mathrm{CDCl}_{3}\right.$ ) of compound 42 (containing residual ethyl acetate). 


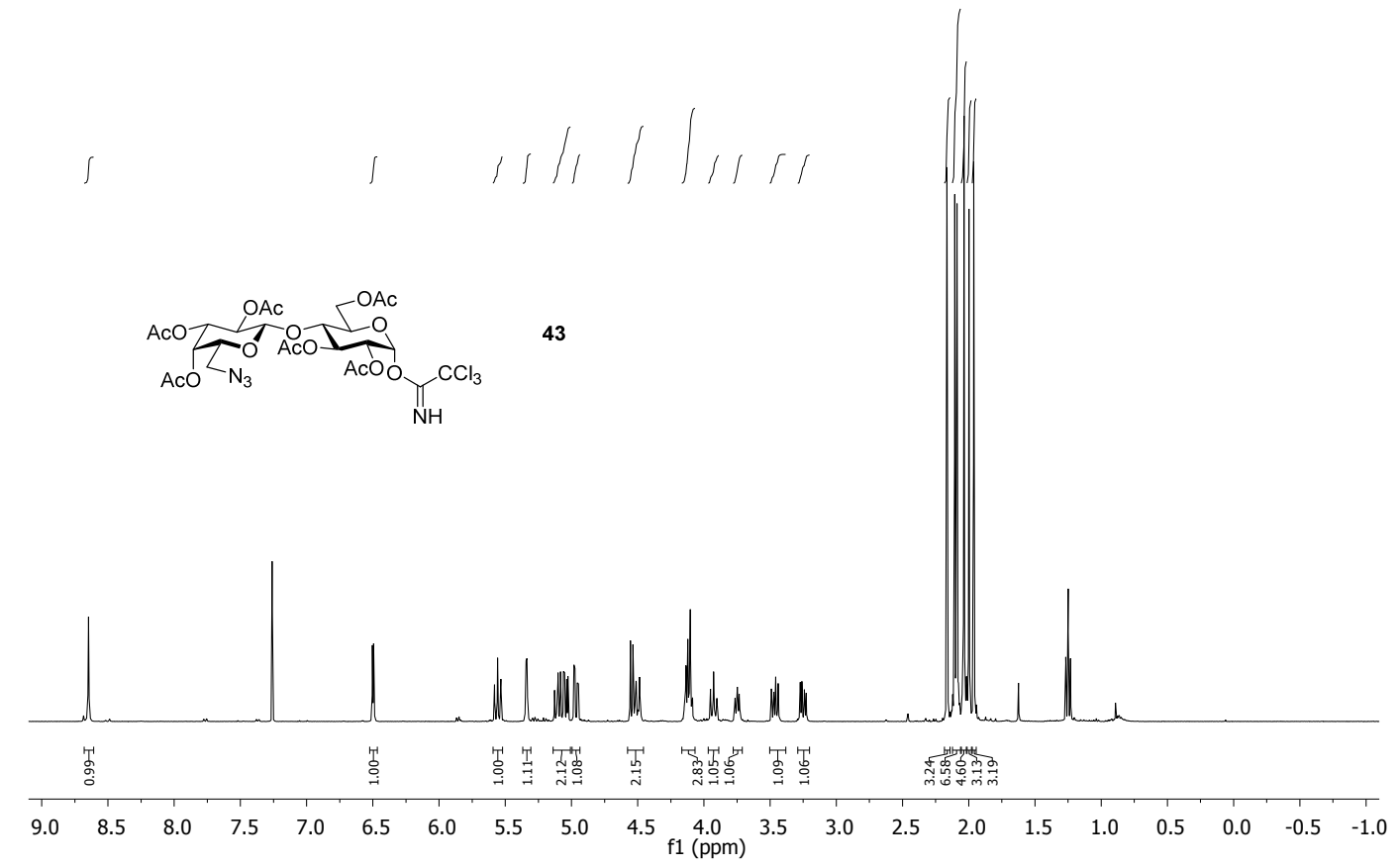

${ }^{1} \mathrm{H}$ NMR spectrum (400 MHz, $\mathrm{CDCl}_{3}$ ) of compound $\mathbf{4 3}$ (containing residual ethyl acetate).

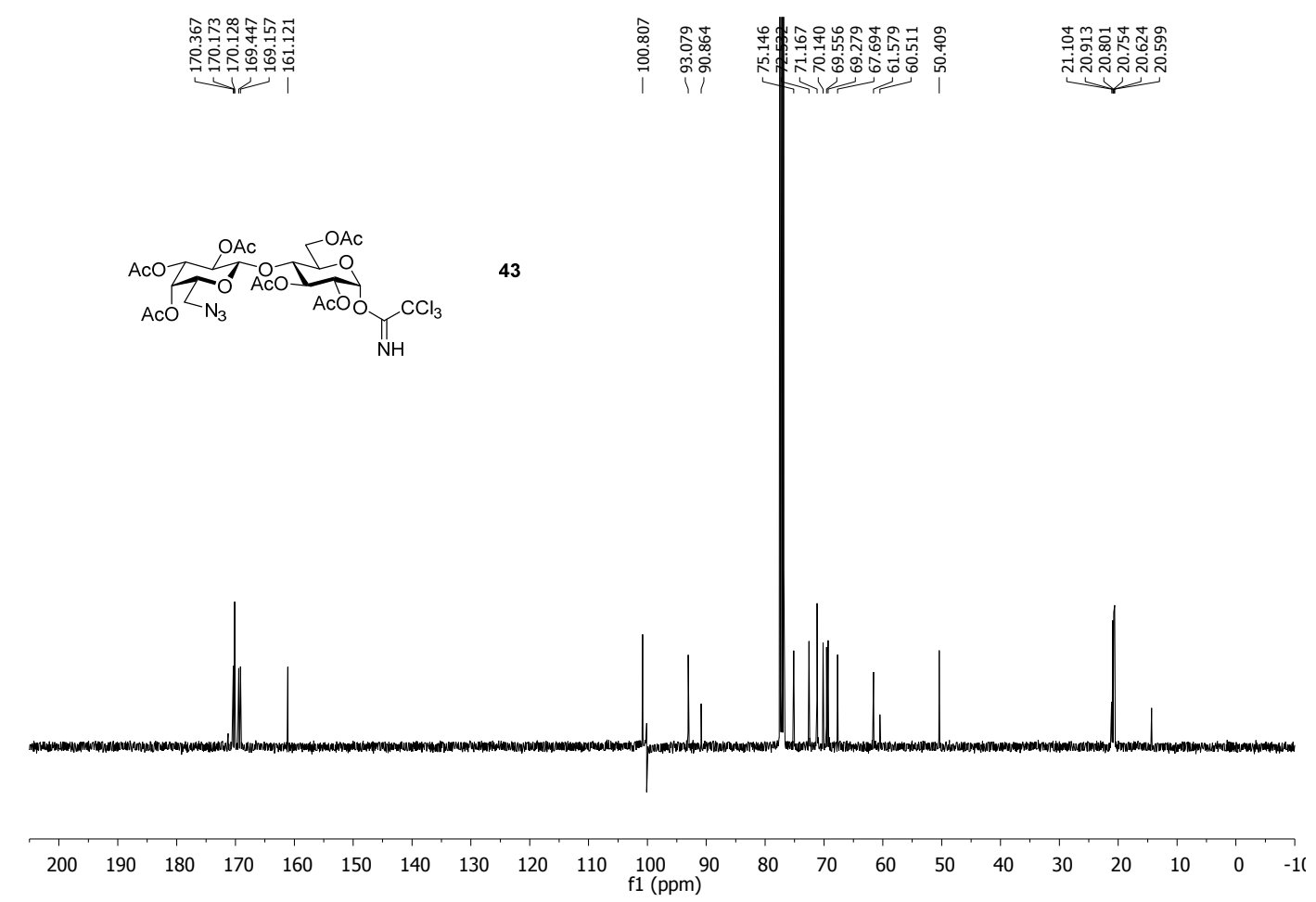

${ }^{13} \mathrm{C}$ NMR spectrum $\left(100.6 \mathrm{MHz}, \mathrm{CDCl}_{3}\right.$ ) of compound 43 (containing residual ethyl acetate). 


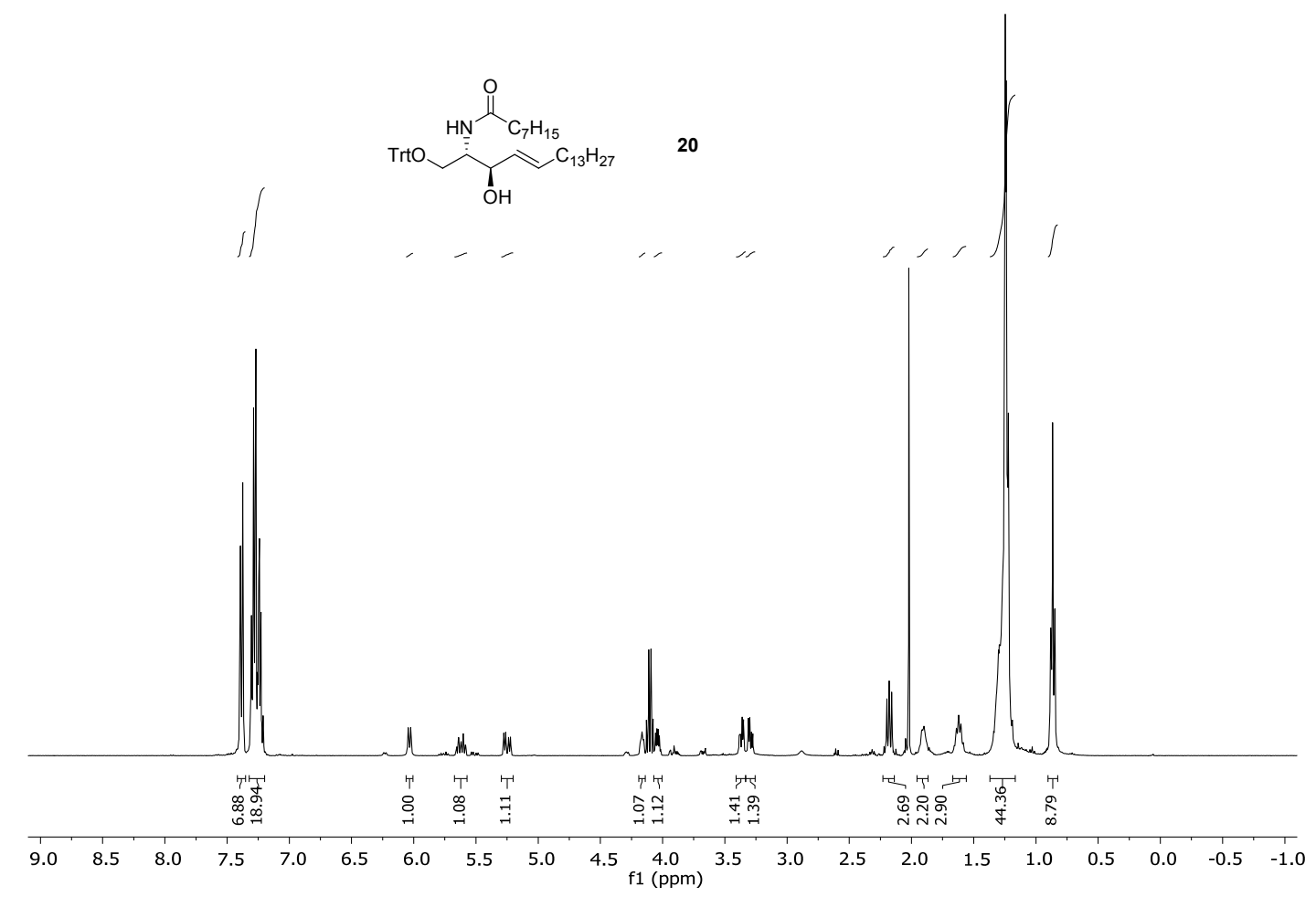

${ }^{1} \mathrm{H}$ NMR spectrum (400 MHz, $\mathrm{CDCl}_{3}$ ) of compound $\mathbf{2 0}$ (containing residual ethyl acetate).

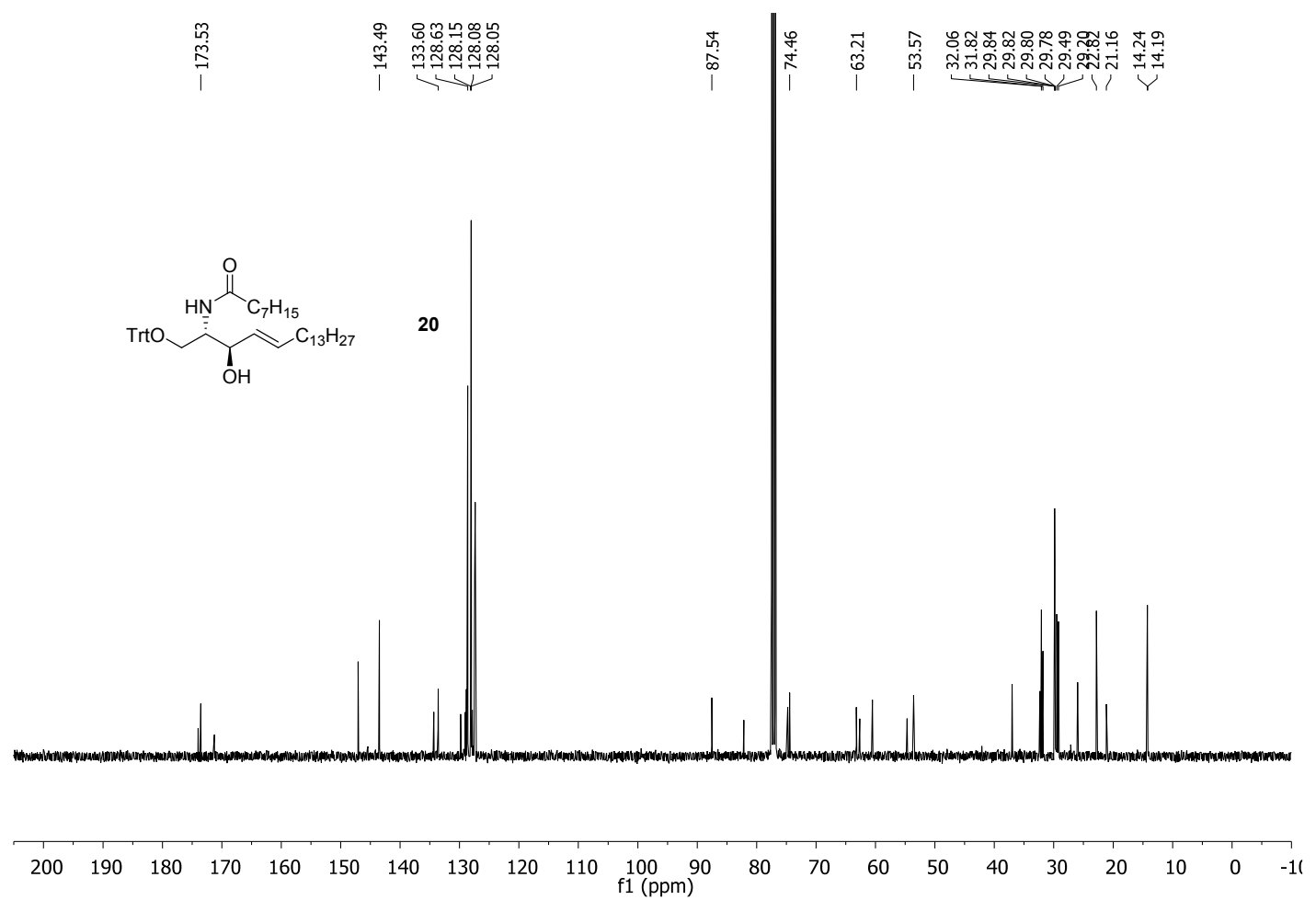

${ }^{13} \mathrm{C}$ NMR spectrum $\left(100.6 \mathrm{MHz}, \mathrm{CDCl}_{3}\right.$ ) of compound $\mathbf{2 0}$ (containing residual ethyl acetate). 


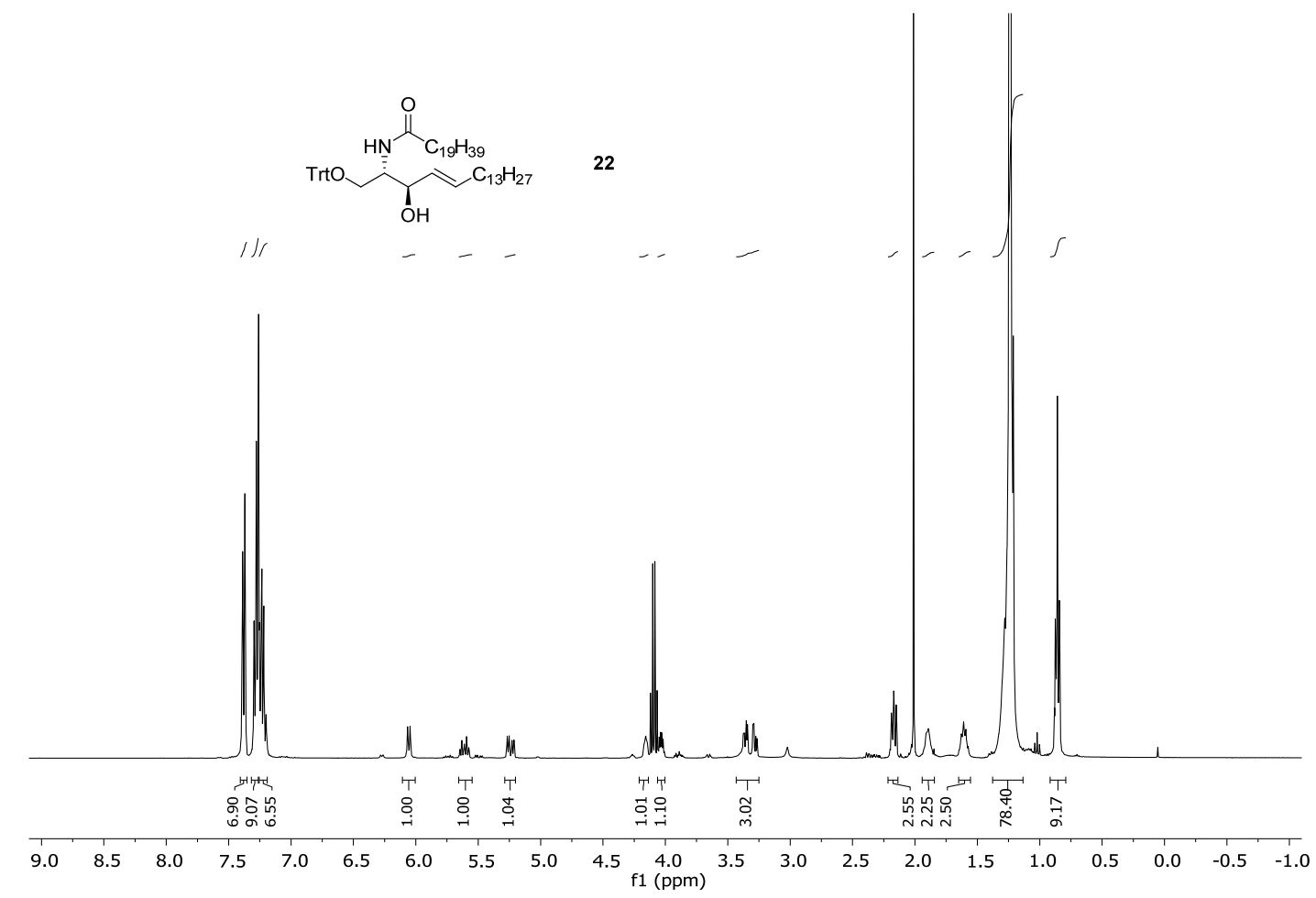

${ }^{1} \mathrm{H}$ NMR spectrum (400 MHz, $\mathrm{CDCl}_{3}$ ) of compound 22 (containing residual ethyl acetate).
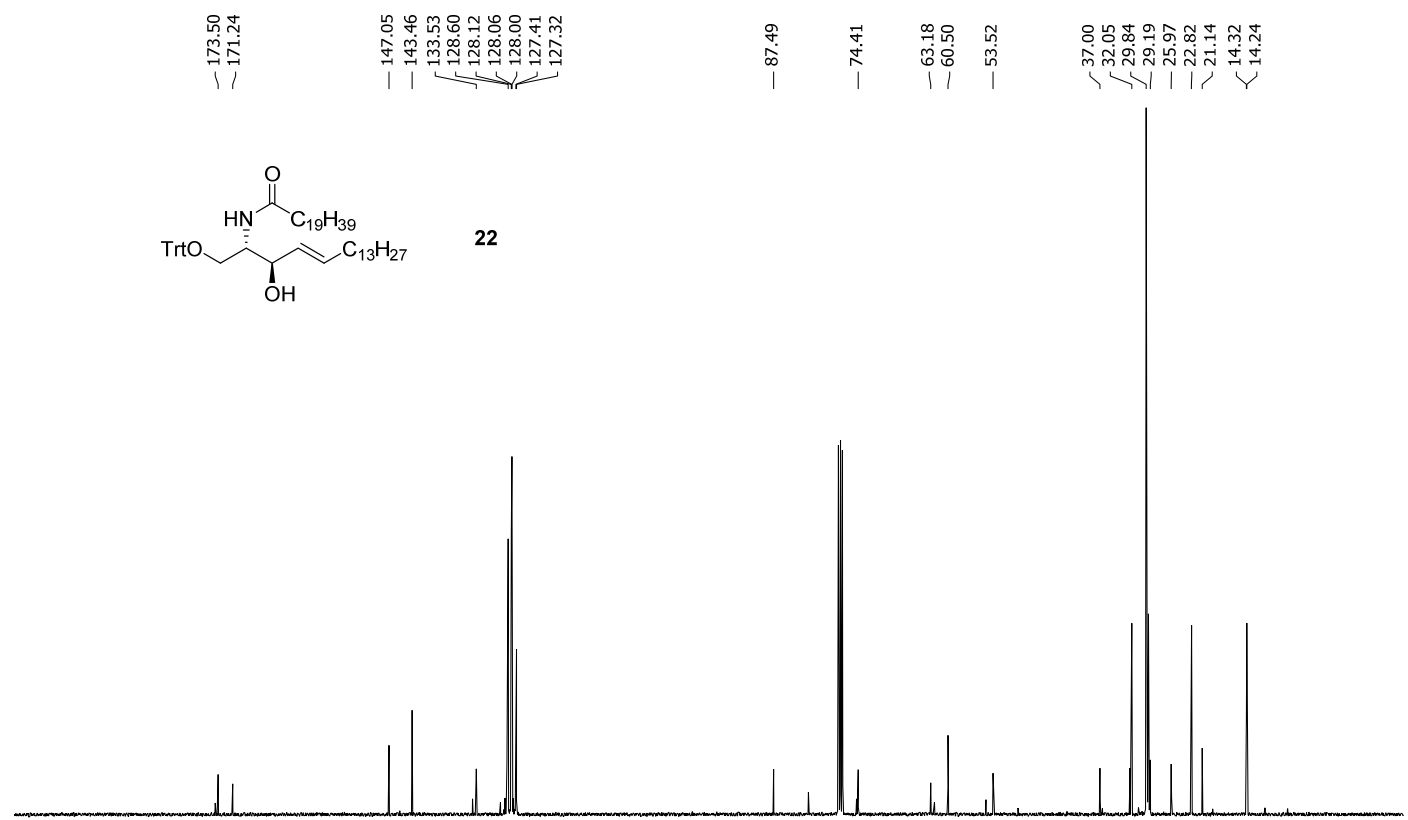

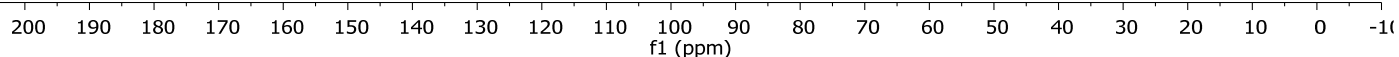

${ }^{13} \mathrm{C}$ NMR spectrum $\left(100.6 \mathrm{MHz}, \mathrm{CDCl}_{3}\right.$ ) of compound 22 (containing residual ethyl acetate). 


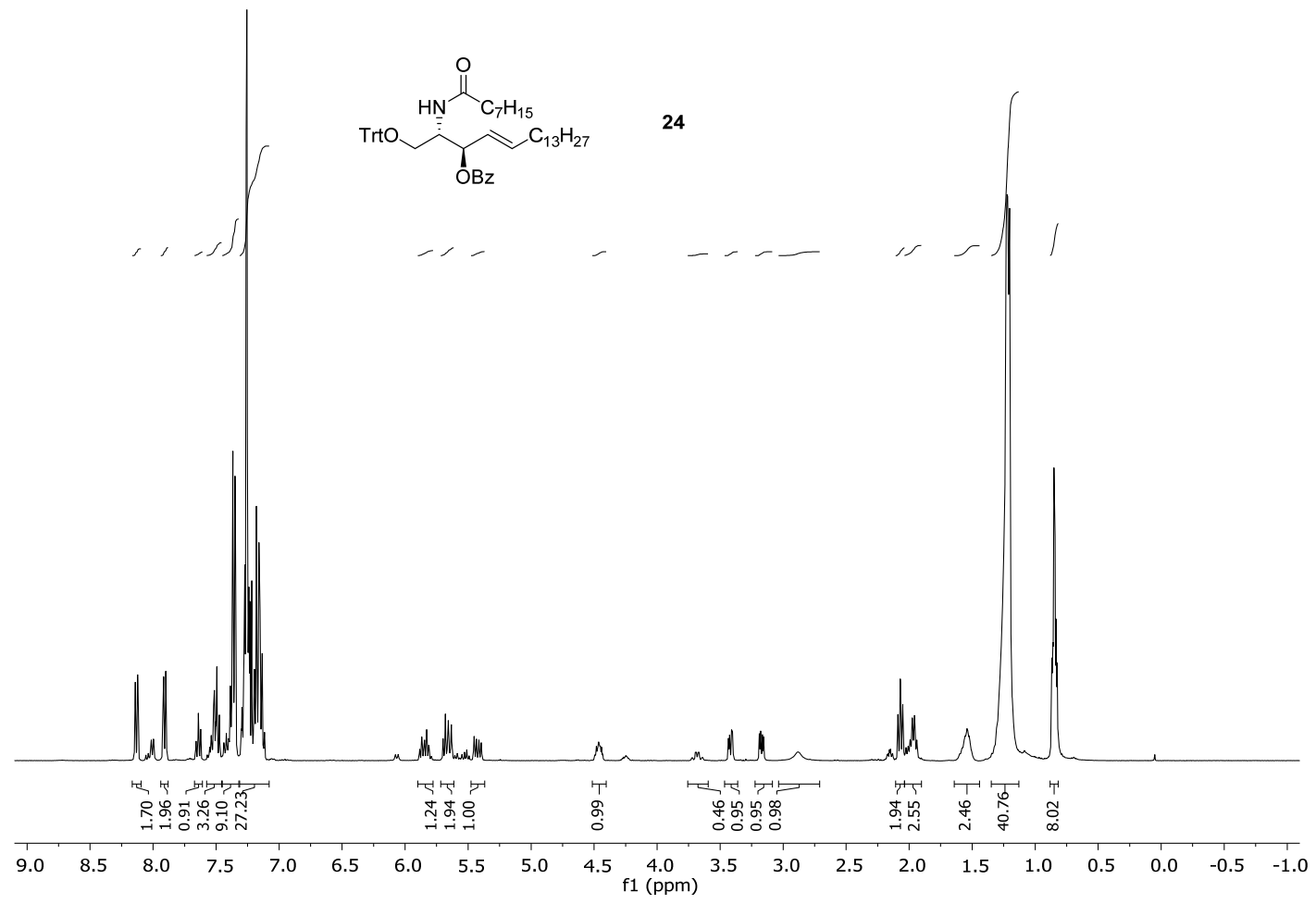

${ }^{1} \mathrm{H}$ NMR spectrum (400 MHz, $\mathrm{CDCl}_{3}$ ) of compound 24.

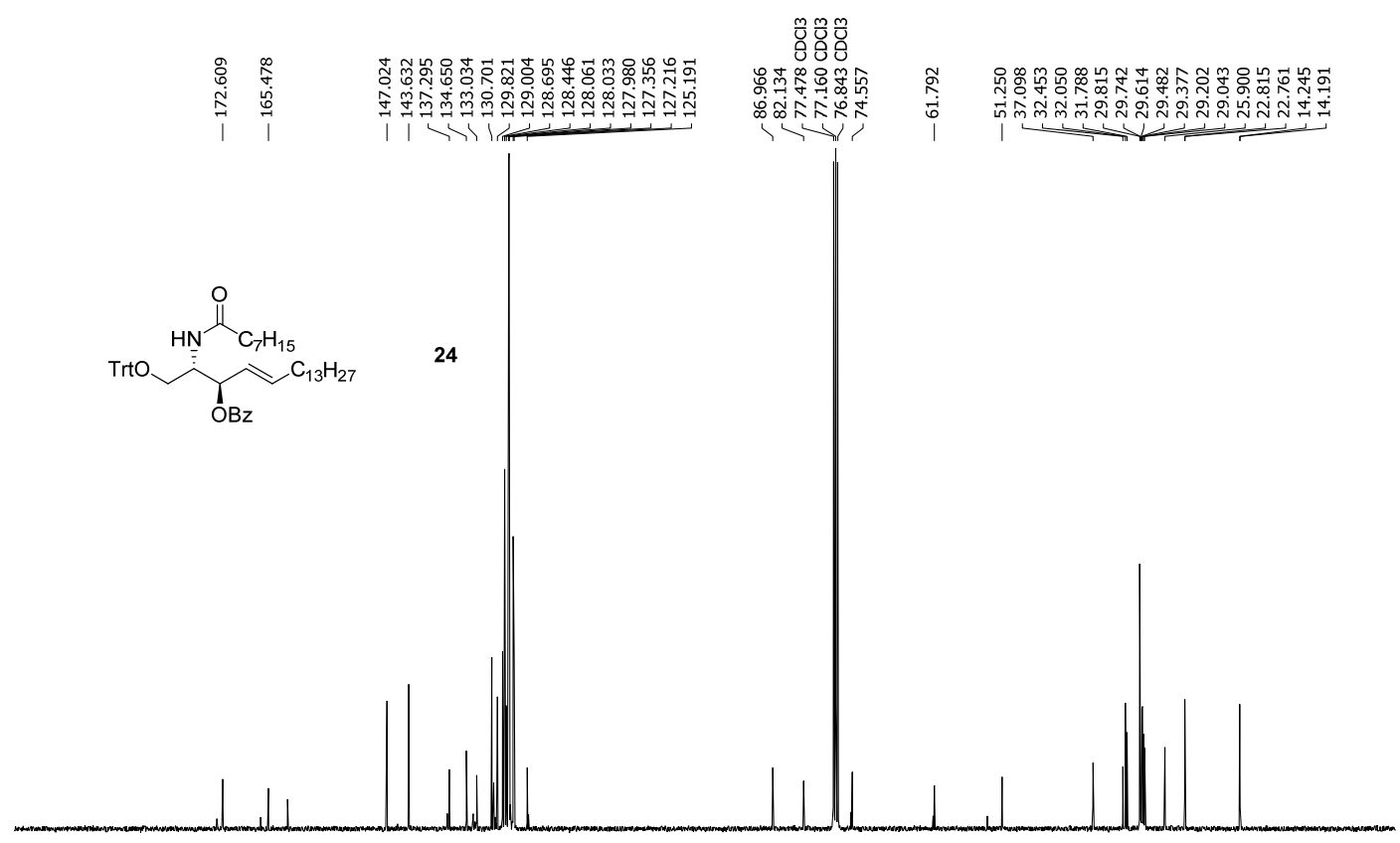

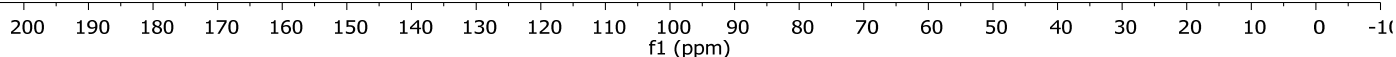

${ }^{13} \mathrm{C}$ NMR spectrum $\left(100.6 \mathrm{MHz}, \mathrm{CDCl}_{3}\right)$ of compound 24. 


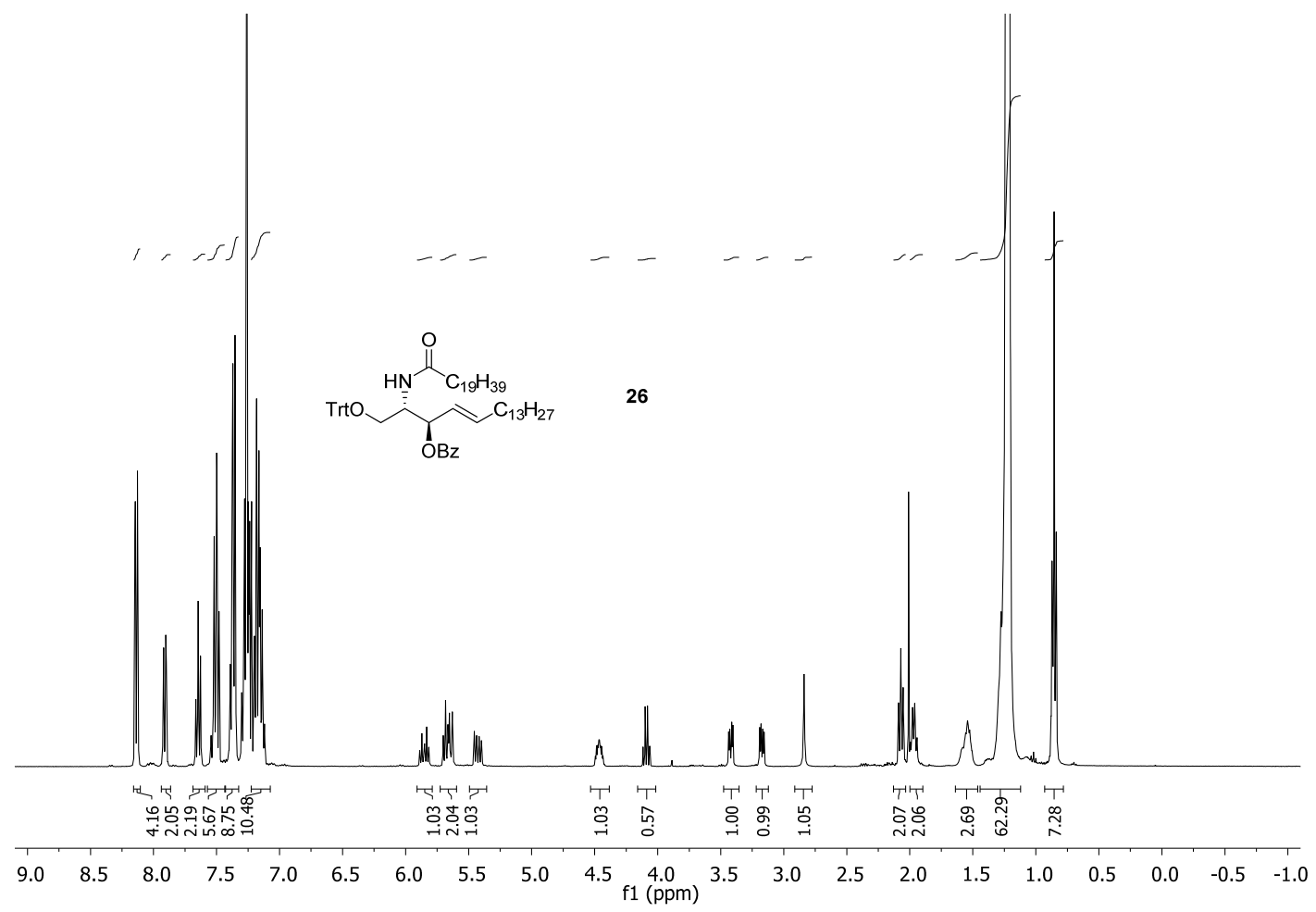

${ }^{1} \mathrm{H}$ NMR spectrum (400 MHz, $\mathrm{CDCl}_{3}$ ) of compound $\mathbf{2 6}$ (containing residual ethyl acetate).

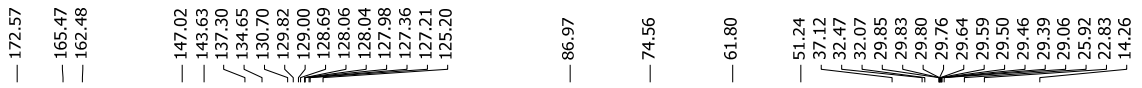
$\underbrace{\mathrm{C}_{\mathrm{Bz}}}_{\mathrm{OBz}}$

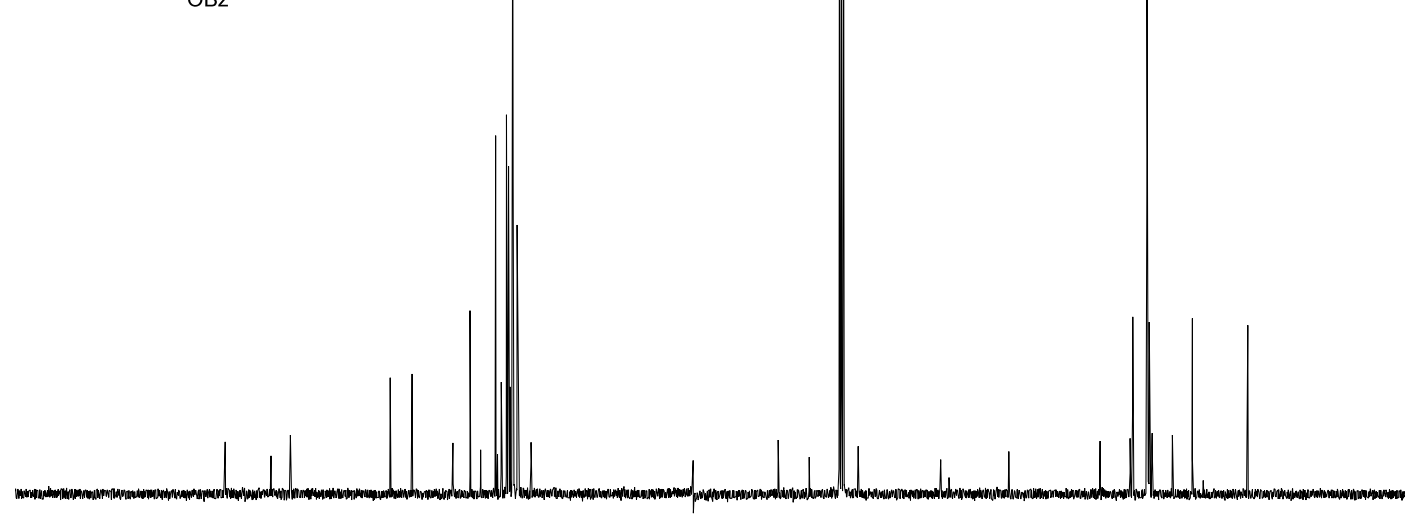

$\begin{array}{llllllllllllllllllllll}200 & 190 & 180 & 170 & 160 & 150 & 140 & 130 & 120 & 110 & 100 & 90 & 80 & 70 & 60 & 50 & 40 & 30 & 20 & 10 & 0 & -11\end{array}$

${ }^{13} \mathrm{C}$ NMR spectrum $\left(100.6 \mathrm{MHz}, \mathrm{CDCl}_{3}\right)$ of compound 26. 


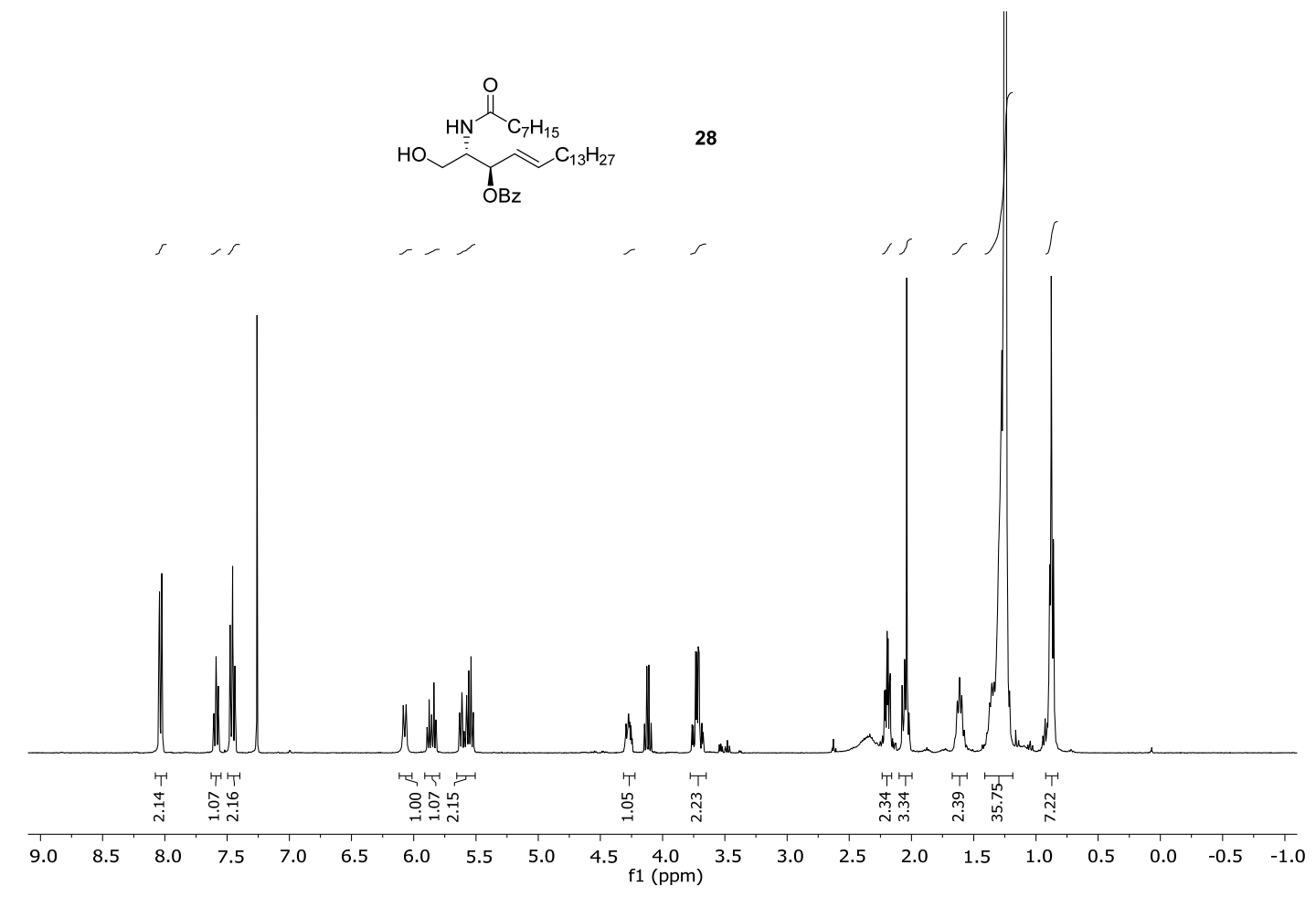

${ }^{1} \mathrm{H}$ NMR spectrum (400 MHz, $\mathrm{CDCl}_{3}$ ) of compound 28 (containing residual ethyl acetate).

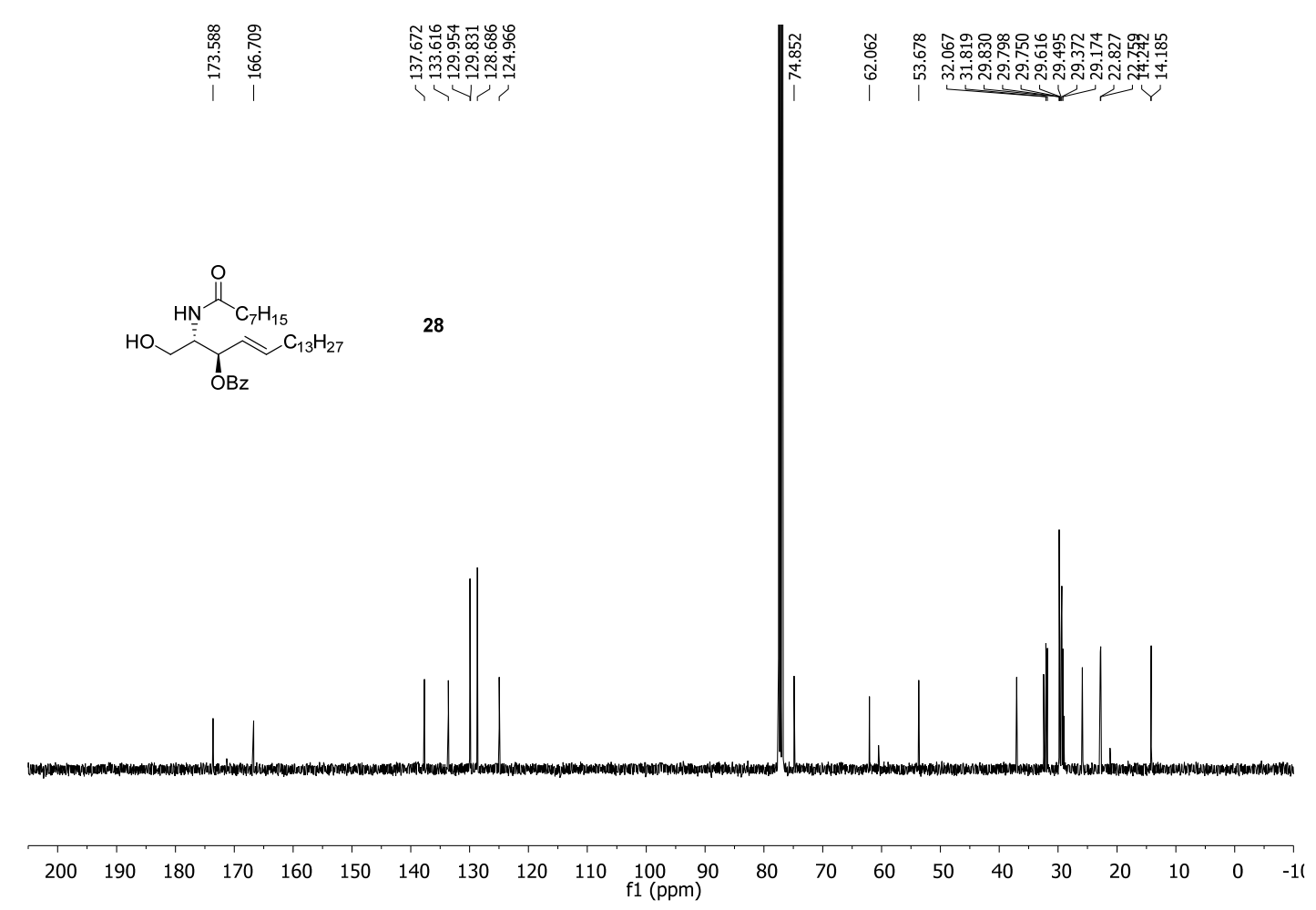

${ }^{13} \mathrm{C}$ NMR spectrum $\left(100.6 \mathrm{MHz}, \mathrm{CDCl}_{3}\right.$ ) of compound 28 (containing residual ethyl acetate). 


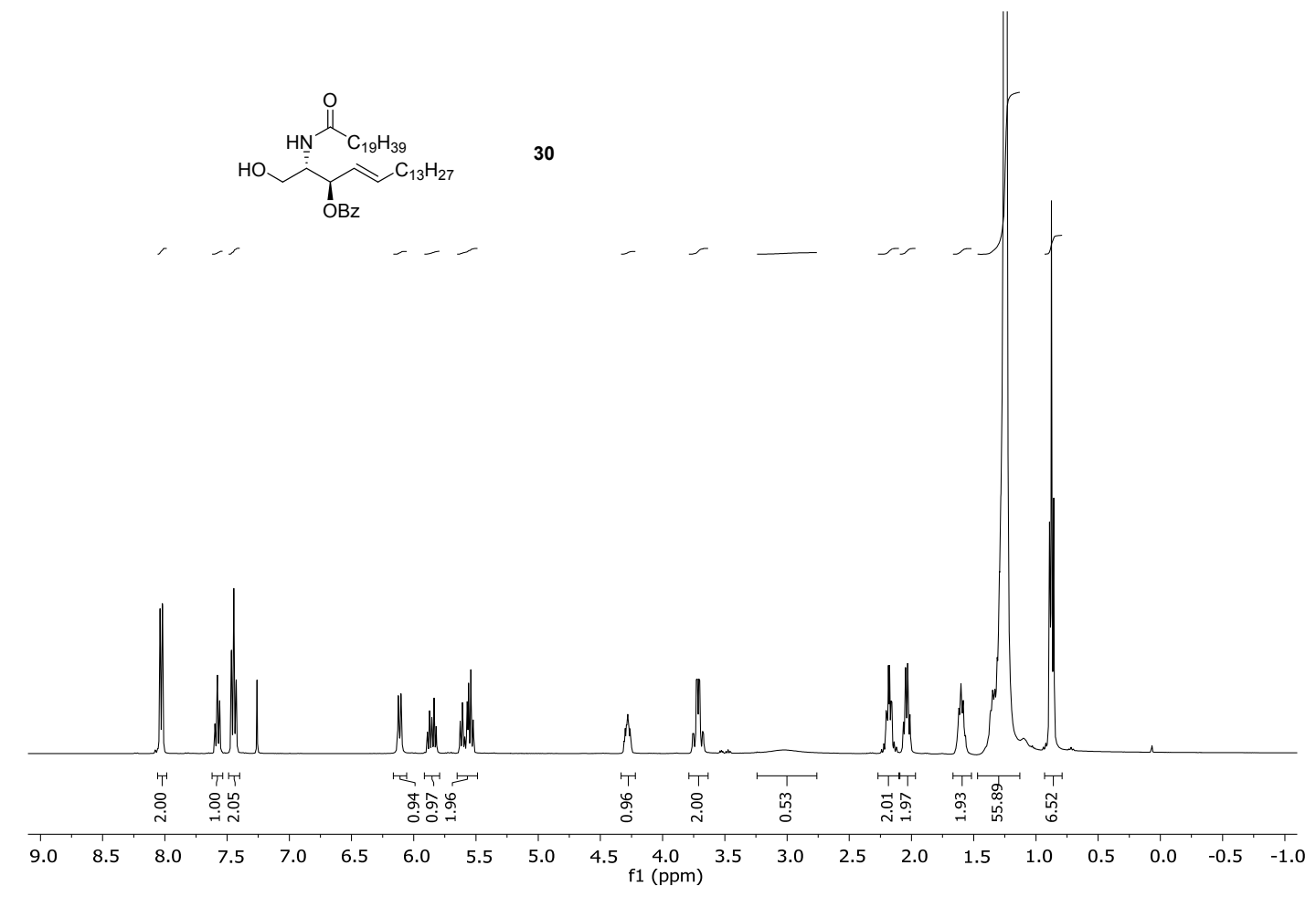

${ }^{1} \mathrm{H}$ NMR spectrum (400 MHz, $\mathrm{CDCl}_{3}$ ) of compound $\mathbf{3 0}$.
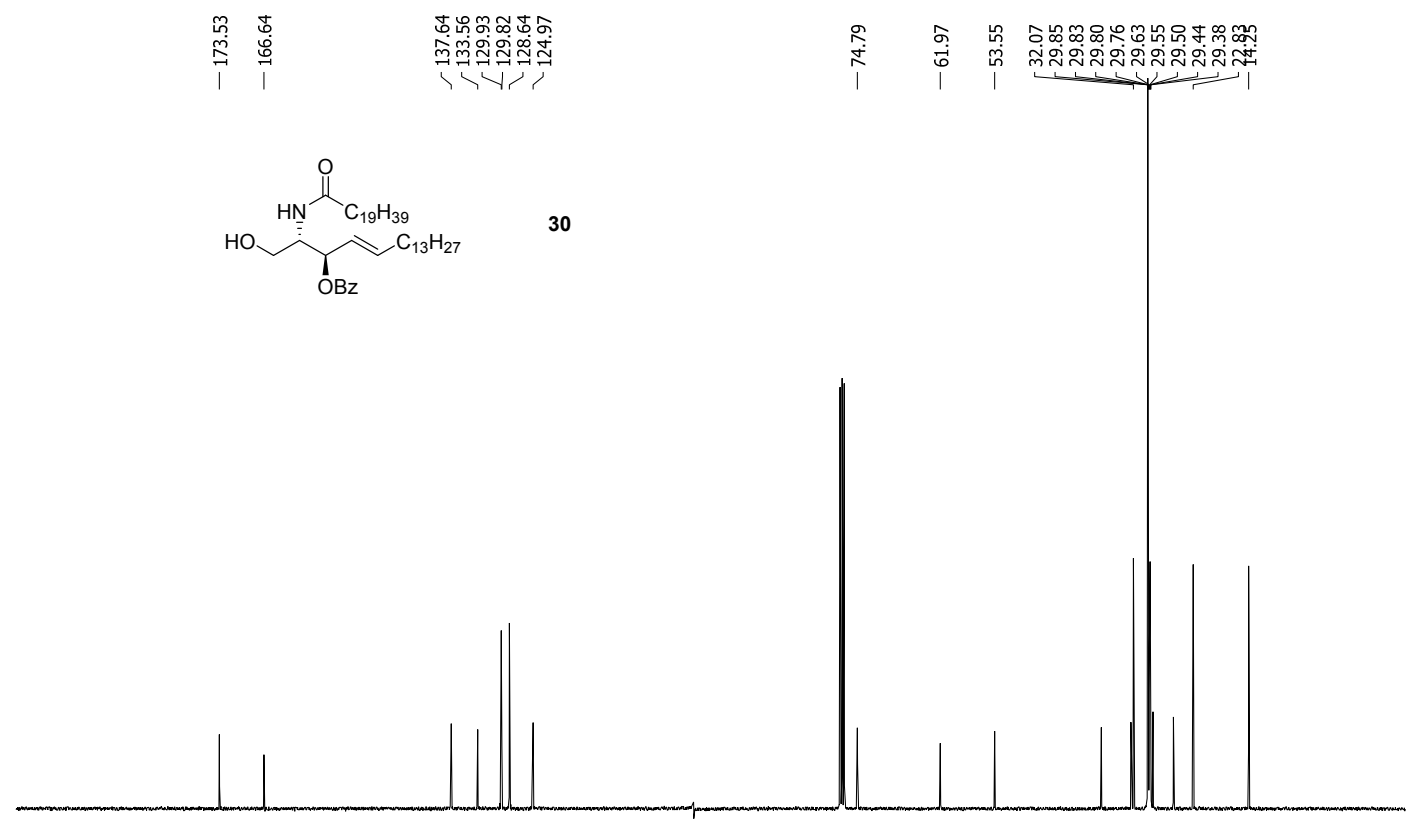

$\begin{array}{lllllllllllllllllllllllllll}200 & 190 & 180 & 170 & 160 & 150 & 140 & 130 & 120 & 110 & 100 & 90 & 80 & 70 & 60 & 50 & 40 & 30 & 20 & 10 & 0 & -11\end{array}$

${ }^{13} \mathrm{C}$ NMR spectrum $\left(100.6 \mathrm{MHz}, \mathrm{CDCl}_{3}\right.$ ) of compound $\mathbf{3 0}$. 


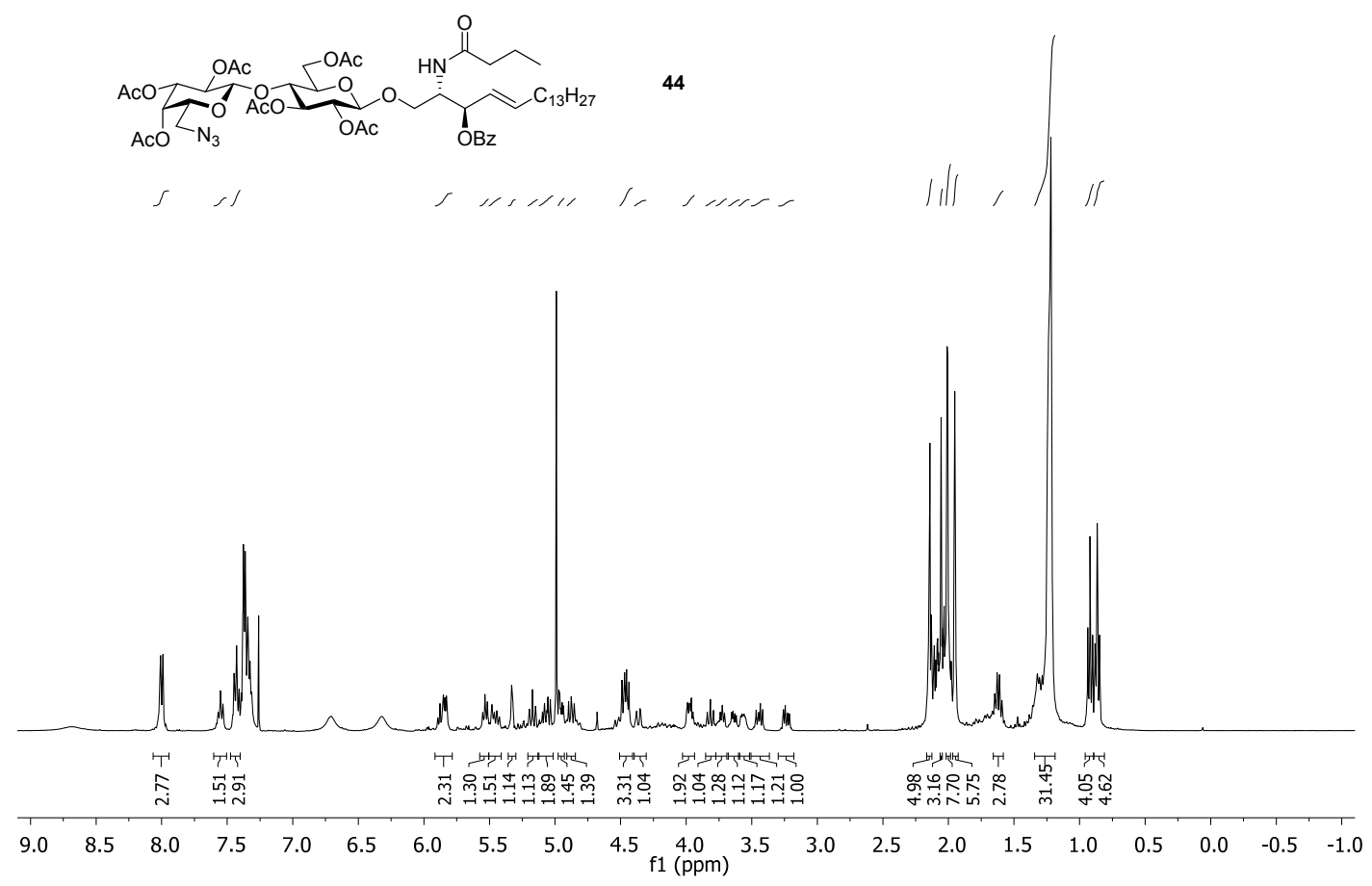

${ }^{1} \mathrm{H}$ NMR spectrum (400 MHz, $\mathrm{CDCl}_{3}$ ) of compound 44 (containing residual trichloroacetamide).

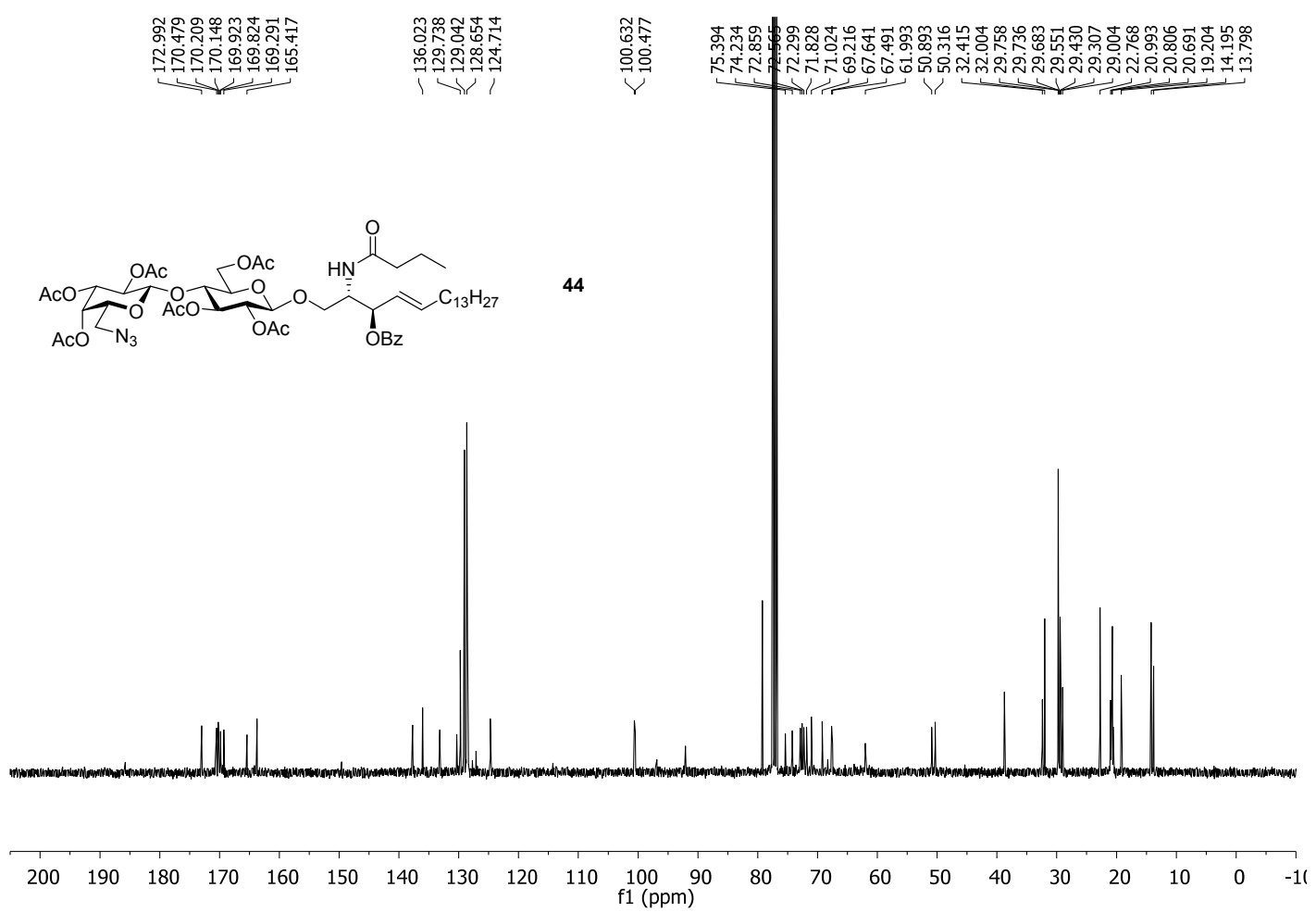

${ }^{13} \mathrm{C}$ NMR spectrum (100.6 MHz, $\mathrm{CDCl}_{3}$ ) of compound 44 (containing residual trichloroacetamide). 


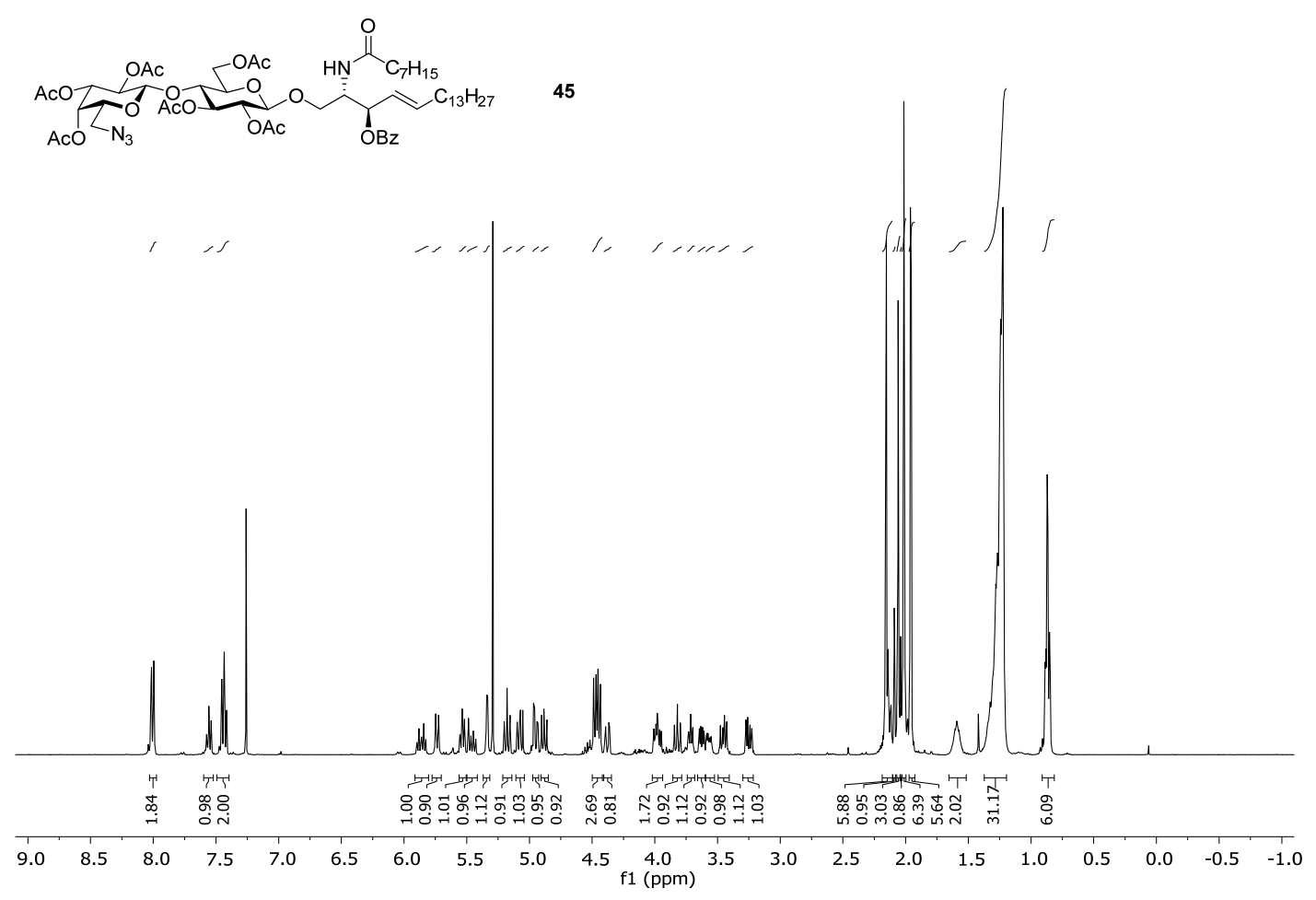

${ }^{1} \mathrm{H}$ NMR spectrum (400 MHz, $\mathrm{CDCl}_{3}$ ) of compound 45.

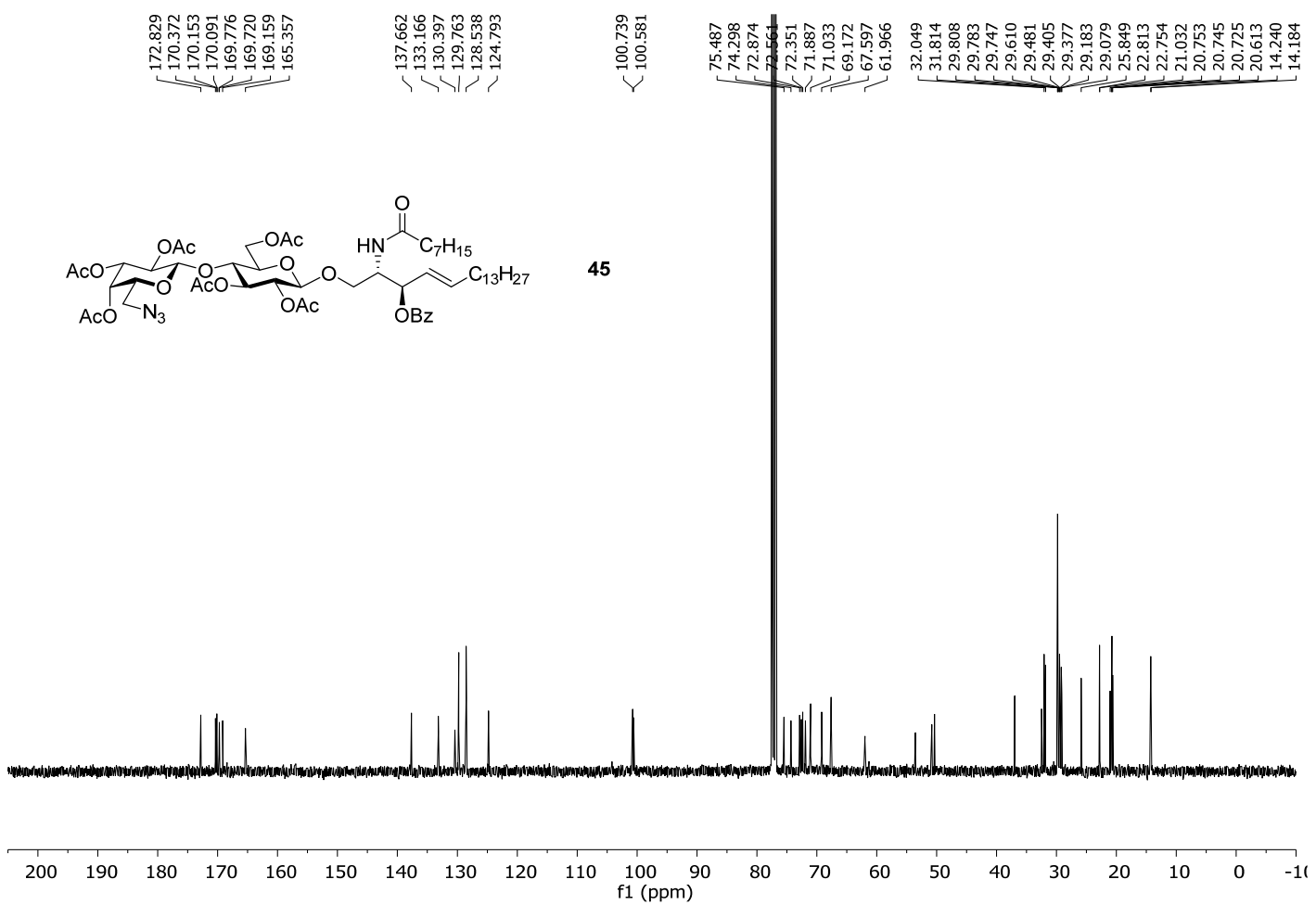

${ }^{13} \mathrm{C}$ NMR spectrum $\left(100.6 \mathrm{MHz}, \mathrm{CDCl}_{3}\right)$ of compound 45. 


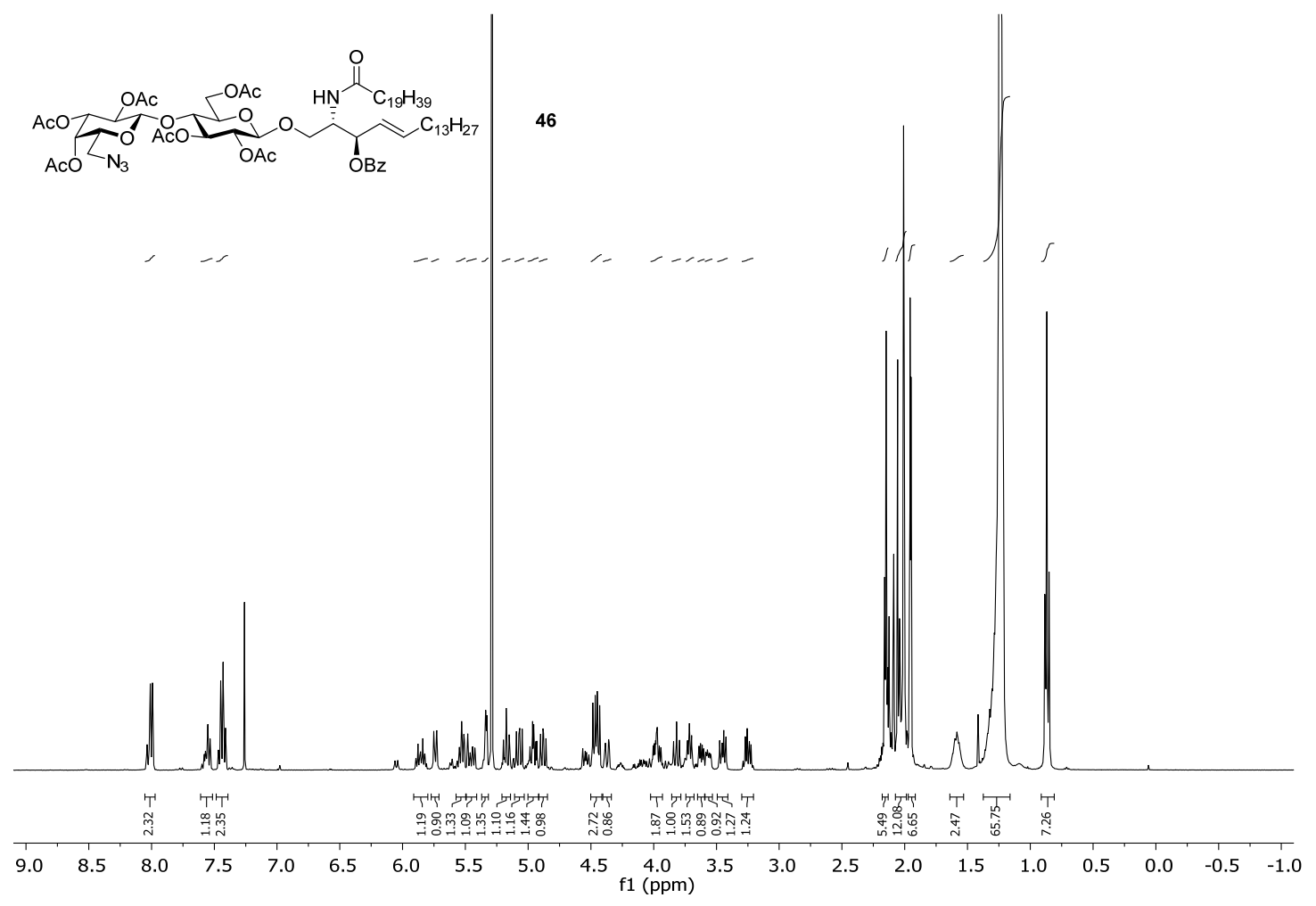

${ }^{1} \mathrm{H}$ NMR spectrum $\left(400 \mathrm{MHz}, \mathrm{CDCl}_{3}\right)$ of compound 46.
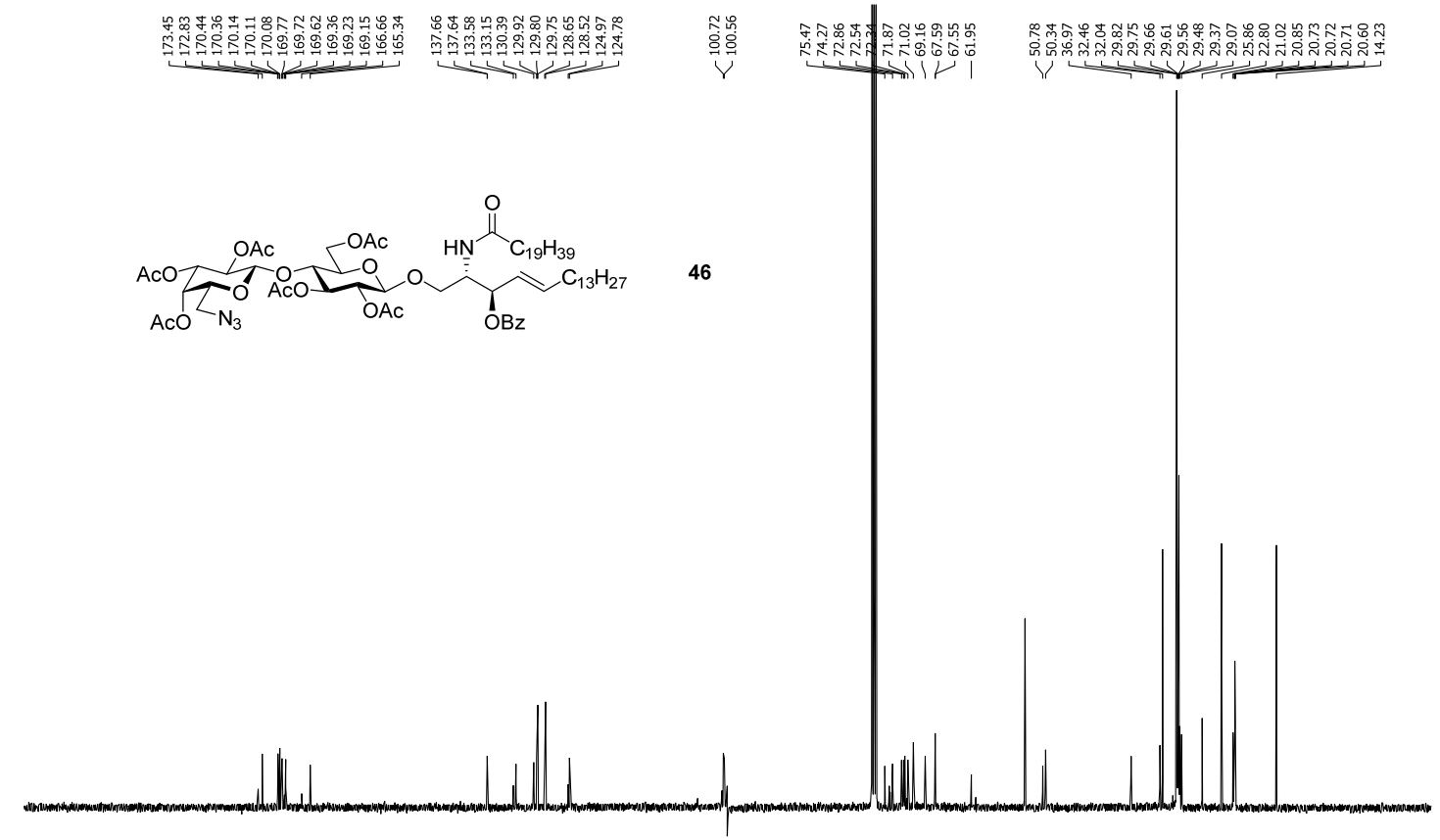

$\begin{array}{lllllllllllllllllllllll}210 & 200 & 190 & 180 & 170 & 160 & 150 & 140 & 130 & 120 & 110 & 100 & 90 & 80 & 70 & 60 & 50 & 40 & 30 & 20 & 10 & 0 & -11\end{array}$

${ }^{13} \mathrm{C}$ NMR spectrum $\left(100.6 \mathrm{MHz}, \mathrm{CDCl}_{3}\right)$ of compound 46. 


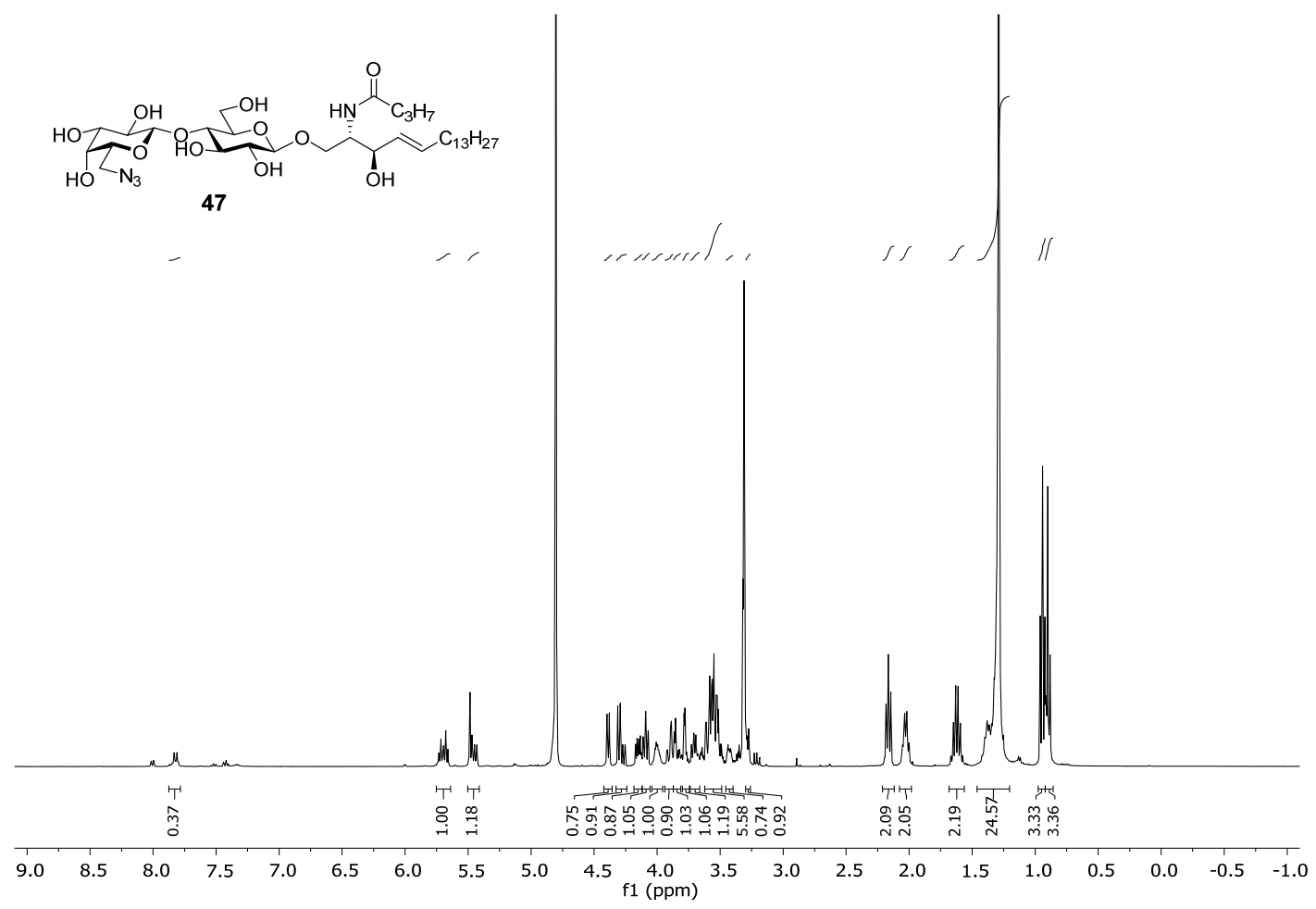

${ }^{1} \mathrm{H}$ NMR spectrum (400 MHz, $\mathrm{CD}_{3} \mathrm{OD}$ ) of compound 47.

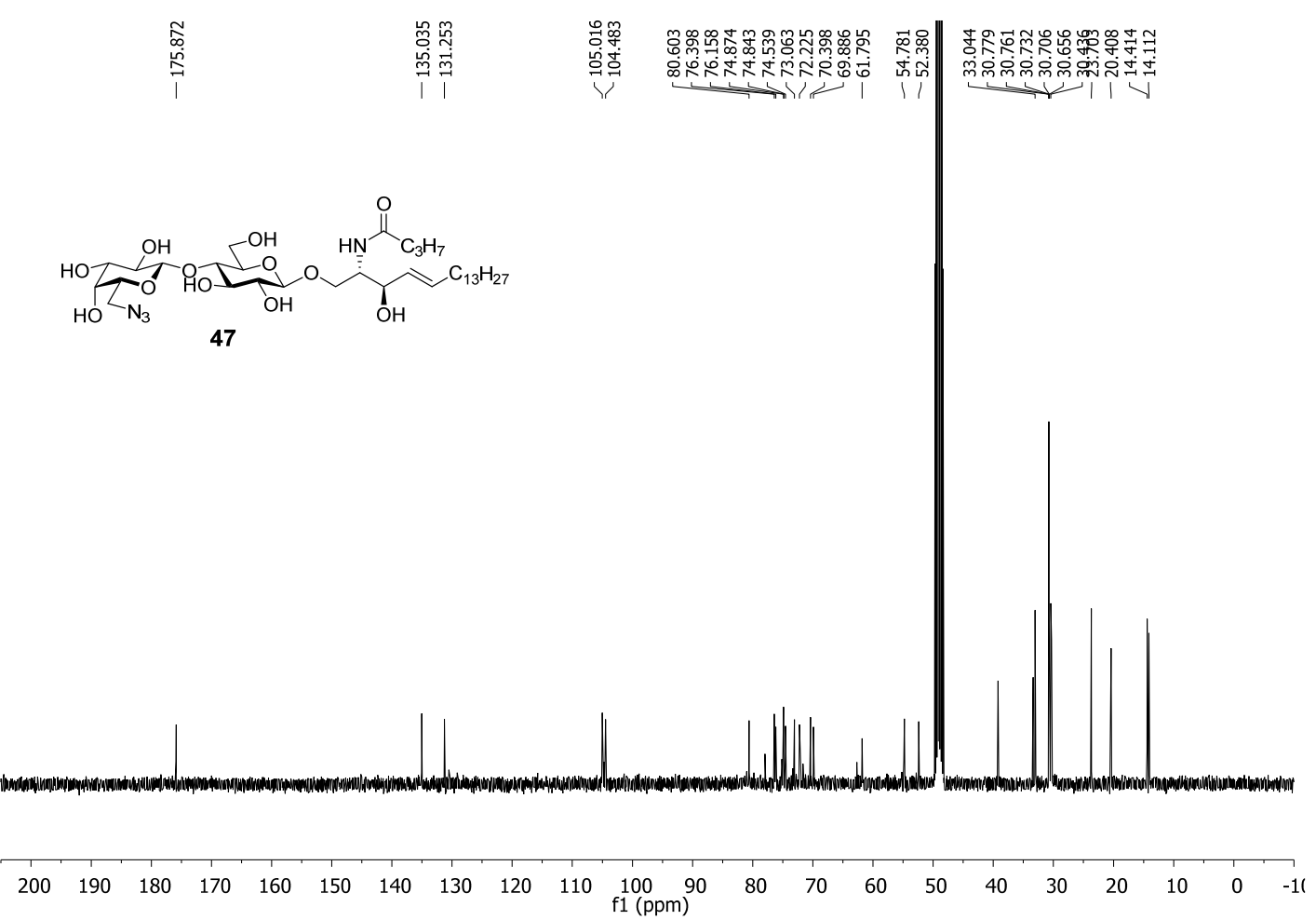

${ }^{13} \mathrm{C}$ NMR spectrum $\left(100.6 \mathrm{MHz}, \mathrm{CD}_{3} \mathrm{OD}\right)$ of compound 47. 


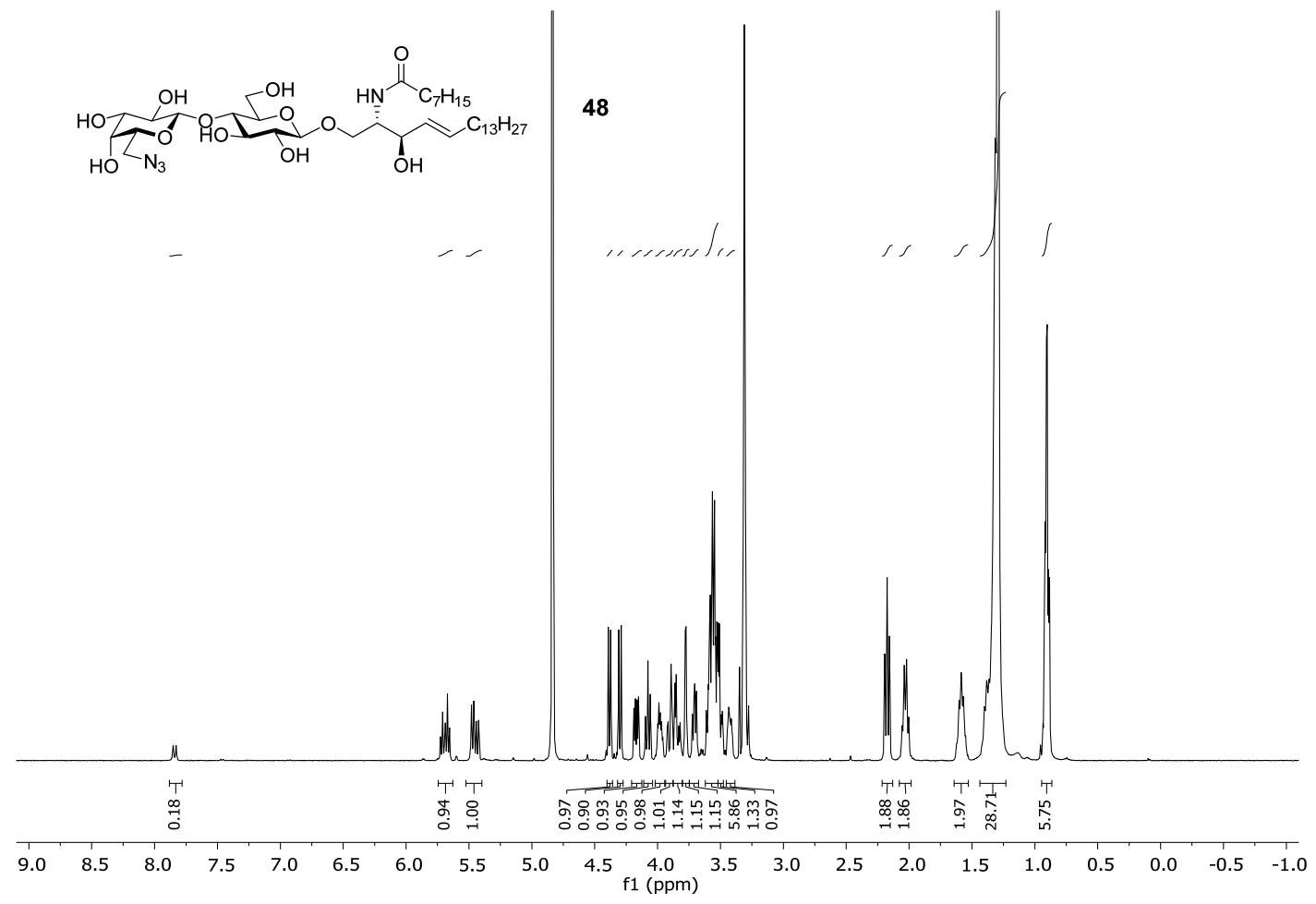

${ }^{1} \mathrm{H}$ NMR spectrum (400 MHz, $\mathrm{CD}_{3} \mathrm{OD}$ ) of compound $\mathbf{4 8}$.

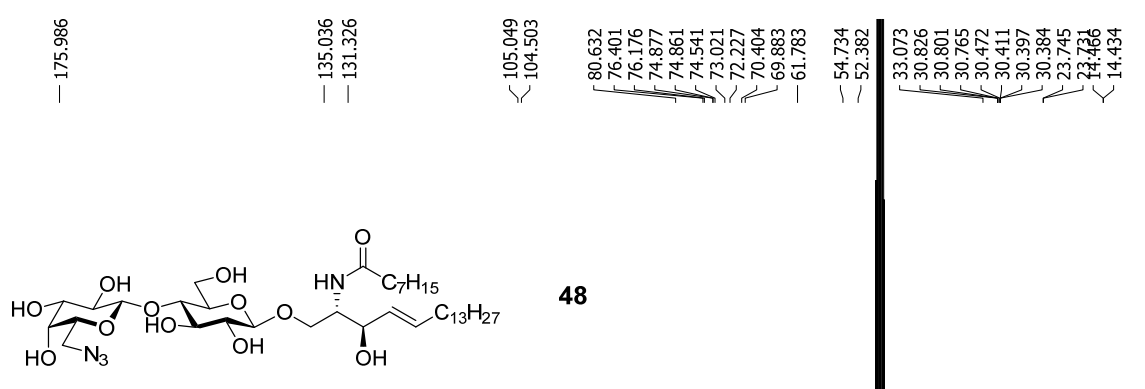

$\begin{array}{llllllllllllllllllllll}200 & 190 & 180 & 170 & 160 & 150 & 140 & 130 & 120 & 110 & \underset{f 1}{100}(\mathrm{ppm}) & 90 & 80 & 70 & 60 & 50 & 40 & 30 & 20 & 10 & 0 & -1(\end{array}$

${ }^{13} \mathrm{C}$ NMR spectrum $\left(100.6 \mathrm{MHz}, \mathrm{CD}_{3} \mathrm{OD}\right)$ of compound 48. 


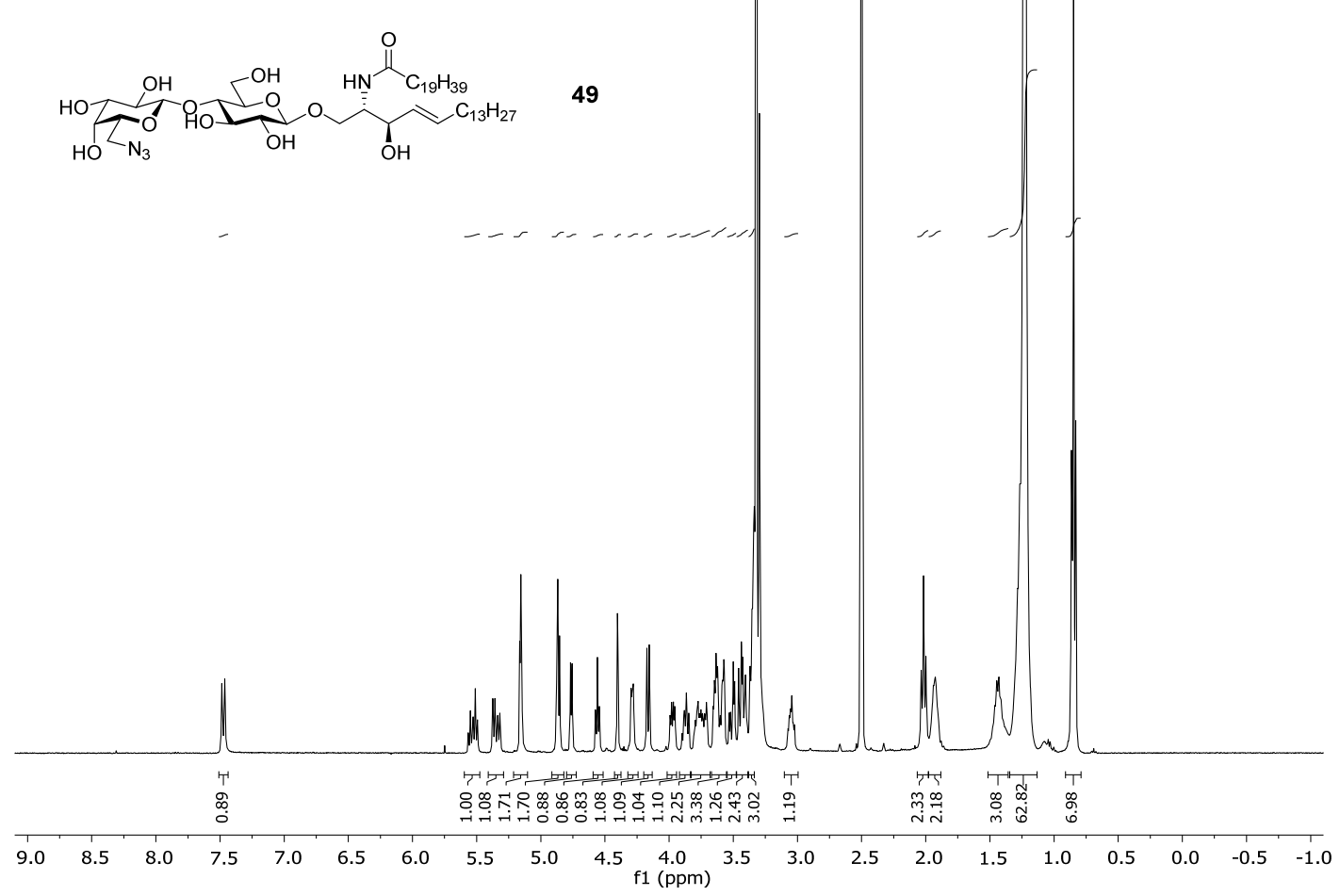

${ }^{1} \mathrm{H}$ NMR spectrum (400 MHz, DMSO) of compound 49.
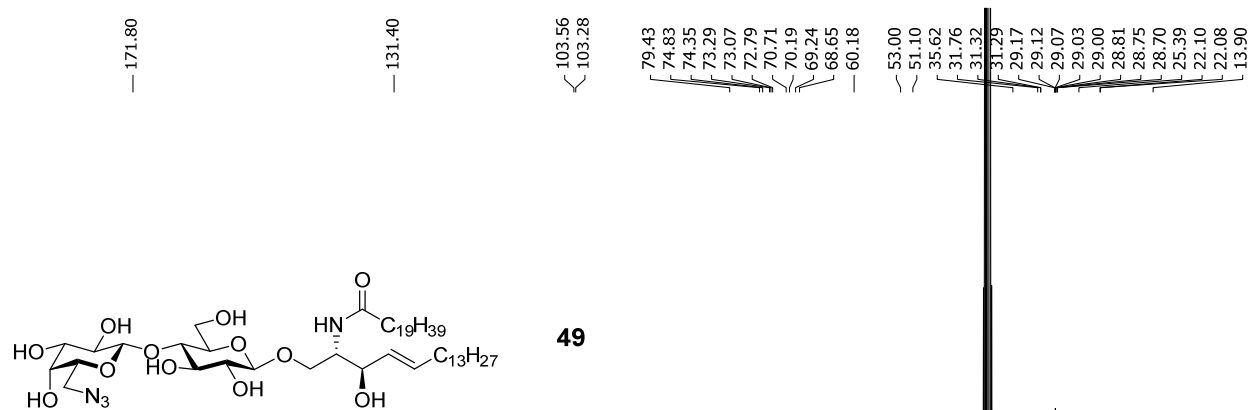

49

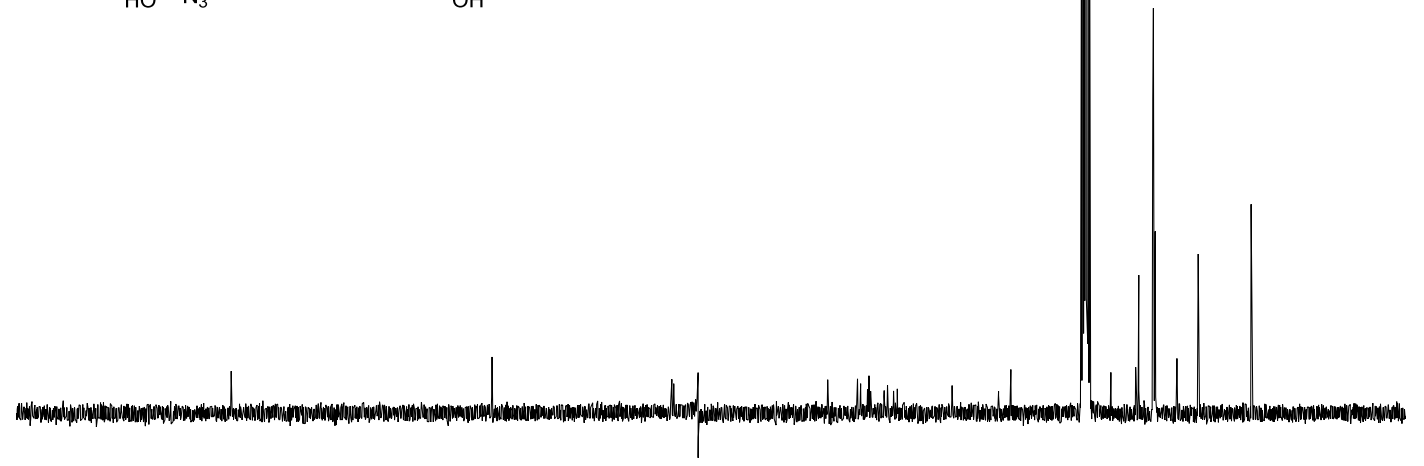

$\begin{array}{llllllllllllllllllllll}200 & 190 & 180 & 170 & 160 & 150 & 140 & 130 & 120 & 110 & \begin{array}{c}100 \\ \mathrm{f} 1(\mathrm{ppm})\end{array} & 80 & 70 & 60 & 50 & 40 & 30 & 20 & 10 & 0 & -11\end{array}$

${ }^{13} \mathrm{C}$ NMR spectrum (100.6 MHz, DMSO) of compound 49. 